\title{
Modeling Belief in Dynamic Systems Part II: Revision and Update
}

Nir Friedman

Institute of Computer Science

Hebrew University, Jerusalem, 91904, ISRAEL

http://www.cs.huji.ac.il/ nir

Joseph Y. Halpern

Computer Science Department

Cornell University, Ithaca, NY 14853

http://www. cs. cornell . edu/home/halpern
NIR@CS.HUJI.AC.IL

HALPERN@CS.CORNELL.EDU

\begin{abstract}
The study of belief change has been an active area in philosophy and AI. In recent years two special cases of belief change, belief revision and belief update, have been studied in detail. In a companion paper (Friedman \& Halpern, 1997), we introduce a new framework to model belief change. This framework combines temporal and epistemic modalities with a notion of plausibility, allowing us to examine the change of beliefs over time. In this paper, we show how belief revision and belief update can be captured in our framework. This allows us to compare the assumptions made by each method, and to better understand the principles underlying them. In particular, it shows that Katsuno and Mendelzon's notion of belief update (Katsuno \& Mendelzon, 1991a) depends on several strong assumptions that may limit its applicability in artificial intelligence. Finally, our analysis allow us to identify a notion of minimal change that underlies a broad range of belief change operations including revision and update.
\end{abstract}

\section{Introduction}

The study of belief change has been an active area in philosophy and artificial intelligence. The focus of this research is to understand how an agent should change her beliefs as a result of getting new information. Two instances of this general phenomenon have been studied in detail. Belief revision (Alchourrón, Gärdenfors, \& Makinson, 1985; Gärdenfors, 1988) focuses on how an agent should change her (set of) beliefs when she adopts a particular new belief. Belief update (Katsuno \& Mendelzon, 1991a), on the other hand, focuses on how an agent should change her beliefs when she realizes that the world has changed. Both approaches attempt to capture the intuition that an agent should make minimal changes in her beliefs in order to accommodate the new belief. The difference is that belief revision attempts to decide what beliefs should be discarded to accommodate a new belief, while belief update attempts to decide what changes in the world led to the new observation. ${ }^{1}$

1. Throughout the paper we use "revision" to refer to AGM's proposal for revision (Alchourrón et al., 1985) not as a generic term for the general approach initiated by AGM; similarly, we use "update" to refer to KM's proposal for update (Katsuno \& Mendelzon, 1991a). 
Belief revision and belief update are two of many possible ways of modeling belief change. In (Friedman \& Halpern, 1997), we introduce a general framework for modeling belief change. We start with the framework for analyzing knowledge in multi-agent systems, introduced in (Halpern \& Fagin, 1989), and add to it a measure of plausibility at each situation. We then define belief as truth in the most plausible situations. The resulting framework is very expressive; it captures both time and knowledge as well as beliefs. Having time allows us to reason in the framework about changes in the beliefs of the agent. It also allows us to relate the beliefs of the agent about the future with her actual beliefs in the future. Knowledge captures in a precise sense the non-defeasible information the agent has about the world, while belief captures the defeasible assumptions implied by her plausibility assessment. The framework allows us to represent a broad spectrum of notions of belief change. In this paper, we focus on how, in particular, belief revision and update can be represented.

We are certainly not the first to provide semantic models for belief revision and update. For example, (Alchourrón et al., 1985; Grove, 1988; Gärdenfors \& Makinson, 1988; Rott, 1991; Boutilier, 1992; de Rijke, 1992) deal with revision, and (Katsuno \& Mendelzon, 1991a; del Val \& Shoham, 1992) deal with update. In fact, there are several works in the literature that capture both using the same machinery (Katsuno \& Satoh, 1991; Goldszmidt \& Pearl, 1996; Boutilier, 1998), and others that simulate belief revision using belief update (Grahne, Mendelzon, \& Rieter, 1992; del Val \& Shoham, 1994). Our approach is different from most in that we do not construct a specific framework to capture one or both of these belief change paradigms. Instead, we start from a natural framework to model how an agent's knowledge changes over time and add to it machinery that captures a defeasible notion of belief.

We believe that our representation offers a number of advantages, and gives a deeper understanding of both revision and update. For one thing, we show that both revision and update can be viewed as proceeding by conditioning on initial prior plausibilities. Thus, our representation emphasizes the role of conditioning as a way of understanding minimal change. Moreover, it shows that that the major differences between revision and update can be understood as corresponding to differences in initial beliefs. For example, revision places full belief on the assumption that the propositions used to describe the world are static, and do not change their truth value over time. By way of contrast, update allows for the possibility that propositions change their truth value over time. However, the family of prior plausibilities that we use to capture update in our framework have the property that they prefer sequences of events where abnormal events occur as late as possible. Because of this property, conditioning in update always "explains" observations by recent changes. The fact that time appears explicitly in our framework allows us to make these issues precise.

In the literature, revision has been viewed as dealing with static worlds (although an agent's beliefs may change, the underlying world about which the agent is reasoning does not) while update has been viewed as dealing with dynamic worlds (see, for example, (Katsuno \& Mendelzon, 1991a)). We believe that the distinction between static and dynamic worlds is somewhat misleading. In fact, what is important for revision is not that the world is static, but that the propositions used to describe the world are static. For example, "At time 0 the block is on the table" is a static proposition, while "The block is on the table" is not, since it implicitly references the current state of affairs. (Note that the assumption that 
the propositions are static is not unique to belief revision. Bayesian updating, for example, makes similar assumptions.) Because we model time explicitly in our framework, we can examine this issue in more detail. In fact, in Section 7 , we show how we relate these two viewpoints. More precisely, given a system, we replace each proposition $p$ used in the system by a family of propositions " $p$ is true at time $m$ ", one for each time $m$. The resulting system describes exactly the same process as the original system, but from a different linguistic perspective. As we show, if the original system corresponds to KM update, then the resulting system is very close to satisfying the requirements of AGM revision. The only requirement that is not met is that the prior is totally ordered, or ranked. This requirement, however, has been relaxed in several variants of revision (Katsuno \& Mendelzon, 1991b; Rott, 1992). Thus, a large part of the difference between revision and update can be understood as a difference in the language used to describe what is happening.

The generality of our framework forces us to be clear about the assumptions we make in the process of capturing revision and update. As a consequence, we have to deal with issues that have been largely ignored by previous semantic accounts. One of these issues is the status of observations. As we show below, to capture either revision or update, we have to assume that observations are minimally informative - the only information carried by an observation of $\varphi$ is that $\varphi$ should be believed. This is a strong assumption, since most observations carry additional information. For example, when trekking in Nepal, one does not expect to observe the weather in Boston. If an agent observes that it is in fact raining in Boston, then this "observation" might well provide extra information about the world (for example, that cable television is available in Nepal). We remark that in (Boutilier, Friedman, \& Halpern, 1998) there is a treatment of revision in our framework where observations are allowed to convey additional information.

Finally, our representation makes it clear how the intuitions of revision and update can be applied in settings where the postulates used to describe them are not sound. For example, we consider situations where they may be irreversible changes (such as death, or breaking a glass vase), and where the agent may perform actions beyond just making observations. Revision and update, as they stand, cannot handle such situations. As we show, our framework allows us to extend them in a natural way so they do.

The rest of the paper is organized as follows. In Section 2, we give an overview of the framework we introduced in (Friedman \& Halpern, 1997). In Section 3, we give a brief review of belief revision and belief update. In Section 4, we define a specific class of structures that embody assumptions that are common to both update and revision. In Section 5, we describe additional assumptions that are required to capture revision. In Section 6, we describe the assumptions that are required to capture update. In Section 7, we reexamine the differences and similarities between belief revision and update. In Section 8, we consider possible extensions to the setup of revision and update, and discuss how these extensions can be handled in our framework. Finally, in Section 9, we conclude with a discussion of related and future work. 


\section{The Framework}

We now review the framework of Halpern and Fagin (1989) for modeling knowledge, and our extension of it for dealing with belief change. The reader is encouraged to consult (Fagin, Halpern, Moses, \& Vardi, 1995) for further details and motivation.

\subsection{Modeling Knowledge}

The framework of Halpern and Fagin was developed to model knowledge in distributed (i.e., multi-agent) systems (Halpern \& Fagin, 1989; Fagin et al., 1995). In this paper, we restrict our attention to the single agent case. The key assumption in this framework is that we can characterize the system by describing it in terms of a state that changes over time. Formally, we assume that at each point in time, the agent is in one of a possibly infinite set of (local) states. At this point, we do not put any further structure on these states (although, as we shall see from our examples, when we model situations in a natural way, states typically do have a great deal of meaningful structure). Intuitively, this local state encodes the information the agent has observed thus far. There is also an environment, whose state encodes relevant aspects of the system that are not part of the agent's local state.

A global state is a pair $\left(s_{e}, s_{a}\right)$ consisting of the environment state $s_{e}$ and the local state $s_{a}$ of the agent. A run of the system is a function from time (which, for ease of exposition, we assume ranges over the natural numbers) to global states. Thus, if $r$ is a run, then $r(0), r(1), \ldots$ is a sequence of global states that, roughly speaking, is a complete description of what happens over time in one possible execution of the system. Given a run $r$, we can define two functions $r_{e}$ and $r_{a}$ that map from time to states of the environment and the agent, respectively, by taking $r_{e}(m)$ to be the state of the environment in the global state $r(m)$ and $r_{a}(m)$ to be the agent's local state in $r(m)$. We can thus identify run $r$ with the pair of functions $\left\langle r_{e}, r_{a}\right\rangle$. We take a system to consist of a set of runs. Intuitively, these runs describe all the possible behaviors of the system, that is, all the possible sequences of events that could occur in the system over time.

Given a system $\mathcal{R}$, we refer to a pair $(r, m)$ consisting of a run $r \in \mathcal{R}$ and a time $m$ as a point. We say two points $(r, m)$ and $\left(r^{\prime}, m^{\prime}\right)$ are indistinguishable to the agent, and write $(r, m) \sim_{a}\left(r^{\prime}, m^{\prime}\right)$, if $r_{a}(m)=r_{a}^{\prime}\left(m^{\prime}\right)$, i.e., if the agent has the same local state at both points. Finally, an interpreted system $\mathcal{I}$ is a tuple $(\mathcal{R}, \pi)$ consisting of a system $\mathcal{R}$ together with a mapping $\pi$ that associates with each point a truth assignment to a set $\Phi$ of primitive propositions. In an interpreted system we can talk about an agent's knowledge: the agent knows $\varphi$ at a point $(r, m)$ if $\varphi$ holds in all points $\left(r^{\prime}, m^{\prime}\right)$ such that $(r, m) \sim_{a}\left(r^{\prime}, m^{\prime}\right)$. Intuitively, an agent knows $\varphi$ at $(r, m)$ if $\varphi$ is implied by the information in the local state $r_{a}(m)$. We give formal semantics for a language of knowledge (and time and plausibility) in Section 2.3.

Example 2.1: The circuit diagnosis problem has been well studied in the literature (see (Davis \& Hamscher, 1988) for an overview). Consider a circuit that contains $n$ logical components $c_{1}, \ldots, c_{n}$ and $k$ lines $l_{1}, \ldots, l_{k}$. The agent can set the values on the input lines of the circuit and observe the values on the output lines. The agent then compares the actual output values to the expected output values and attempts to locate faulty components. 
Since a single test is usually insufficient to locate the problem, the agent might perform a sequence of such tests.

We want to model diagnosis using an interpreted system. To do so, we need to describe the agent's local state, the state of the environment, and some appropriate propositions for reasoning about diagnosis. Intuitively, the agent's state is the sequence of input-output relations observed, while the environment's state describes the current state of the circuit. This consists of the failure set, that is, the set of faulty components of the circuit and the values on all the lines in the circuit. Each run describes the results of a specific series of tests the agent performs and the results she observes. We make two additional assumptions: (1) the agent does not forget what tests were performed and their results, and (2) the faults are persistent and do not change over time.

To make this precise, we define the environment state at a point $(r, m)$ to consist of the failure set at $(r, m)$, which we denote fault $(r, m)$, as well as the values of all the lines in the circuit. We require that the environment state be consistent with the description of the circuit. Thus, for example, if $c_{1}$ is an AND gate with input lines $l_{1}$ and $l_{2}$ and output line $l_{3}$, then if $r_{e}(m)$ says that $c_{1}$ is not faulty, then we require that there is a 1 on $l_{3}$ if and only if there is a 1 on both $l_{1}$ and $l_{2} \cdot{ }^{2}$ We capture the assumption that faults are persistent by requiring that $\operatorname{fault}(r, m)=\operatorname{fault}(r, 0)$. For our later results, it is useful to describe the agent's observations using our logical language. Consider the set $\Phi_{\text {diag }}=\left\{f_{1}, \ldots, f_{n}, h_{1}, \ldots, h_{k}\right\}$ of primitive propositions, where $f_{i}$ denotes that component $i$ is faulty and $h_{i}$ denotes that there is a 1 on line $i$ (that is, line $i$ in a "high" state). An observation is a conjunction of literals of the form $h_{i}$ and $\neg h_{i}$. The agent's state at time $m$ is a sequence of $m$ such observations. Formally, we define the agent's state $r_{a}(m)$ to be $\left\langle o_{1}, \ldots, o_{m}\right\rangle$, where, intuitively, $o_{k}$ is the formula describing the input-output relation observed at time $k$. We use the notation $i o(r, k)$ to denote the formula describing the observation made by the agent at the point $(r, k)$. Given this language, we can define the interpretation $\pi_{\text {diag }}$ in the obvious way. We say that an observation $o$ is consistent with an environment state $r_{e}(m)$ if the states of the input/output lines in $r_{e}(m)$ agree with these in $o$. The system $\mathcal{R}_{\text {diag }}$ consists of all runs $r$ satisfying these requirements in which $i o(r, m)$ is consistent with $r_{e}(m)$ for all times $m$.

Given the system $\left(\mathcal{R}_{\text {diag }}, \pi_{\text {diag }}\right)$, we can examine the agent's knowledge after making a sequence of observations $o_{1}, \ldots, o_{m}$. It is easy to see that the agent knows that the fault set must be one with which all the observations are consistent. However, the agent cannot rule out any of these fault sets. Thus, even if all the observations a re consistent with the circuit being fault-free, the agent does not know that the circuit is fault-free, since there might be a fault that manifests itself only in configurations that have not yet been tested. Of course, the agent might strongly believe that the circuit is fault-free, but we cannot (yet) express this fact in our formalism. The next section rectifies this problem.

2. Note that this means that we can recover the behavior of the circuit (although not necessarily its exact description) by simply looking at the environment state at a point where there are no failures. Of course, if we could have a yet richer environment state that encodes the actual description of the circuit, but this is unnecessary for the analysis we do here. 


\subsection{Plausibility Measures}

Most non-probabilistic approaches to belief change require (explicitly or implicitly) that the agent has some ordering over possible alternatives. For example, the agent might have a preference ordering over possible worlds (Boutilier, 1994b; Grove, 1988; Katsuno \& Mendelzon, 1991b) or an entrenchment ordering over formulas (Gärdenfors \& Makinson, 1988). This ordering dictates how the agent's beliefs change. For example, in (Grove, 1988), the new beliefs are characterized by the most preferred worlds that are consistent with the new observation, while in (Gärdenfors \& Makinson, 1988), beliefs are discarded according to their degree of entrenchment until it is consistent to add the new observation to the resulting set of beliefs. We represent this ordering using plausibility measures, which were introduced in (Friedman \& Halpern, 1995, 1998b). We briefly review the relevant definitions and results here.

Recall that a probability space is a tuple $(W, \mathcal{F}, \operatorname{Pr})$, where $W$ is a set of worlds, $\mathcal{F}$ is an algebra of measurable subsets of $W$ (that is, a set of subsets closed under union and complementation to which we assign probability), and $\mathrm{Pr}$ is a probability measure, that is, a function mapping each set in $\mathcal{F}$ to a number in $[0,1]$ satisfying the well-known probability axioms $(\operatorname{Pr}(\emptyset)=0, \operatorname{Pr}(W)=1$, and $\operatorname{Pr}(A \cup B)=\operatorname{Pr}(A)+\operatorname{Pr}(B)$, if $A$ and $B$ are disjoint $)$.

Plausibility spaces are a direct generalization of probability spaces. We simply replace the probability measure Pr by a plausibility measure Pl, which, rather than mapping sets in $\mathcal{F}$ to numbers in $[0,1]$, maps them to elements in some arbitrary partially ordered set. We read $\mathrm{Pl}(A)$ as "the plausibility of set $A$ ". If $\mathrm{Pl}(A) \leq \mathrm{Pl}(B)$, then $B$ is at least as plausible as $A$. Formally, a plausibility space is a tuple $S=(W, \mathcal{F}, \mathrm{Pl})$, where $W$ is a set of worlds, $\mathcal{F}$ is an algebra of subsets of $W$, and $\mathrm{Pl}$ maps sets in $\mathcal{F}$ to some domain $D$ of plausibility values partially ordered by a relation $\leq_{D}$ (so that $\leq_{D}$ is reflexive, transitive, and anti-symmetric). We assume that $D$ is pointed: that is, it contains two special elements $\top_{D}$, and $\perp_{D}$ such that $\perp_{D} \leq_{D} d \leq_{D} \top_{D}$ for all $d \in D$; we further assume that $\mathrm{Pl}(W)=\top_{D}$ and $\mathrm{Pl}(\emptyset)=\perp_{D}$. As usual, we define the ordering $<_{D}$ by taking $d_{1}<_{D} d_{2}$ if $d_{1} \leq_{D} d_{2}$ and $d_{1} \neq d_{2}$. We omit the subscript $D$ from $\leq_{D},<_{D}, \top_{D}$, and $\perp_{D}$ whenever it is clear from context.

Since we want a set to be at least as plausible as any of its subsets, we require

A1 If $A \subseteq B$, then $\mathrm{Pl}(A) \leq \mathrm{Pl}(B)$.

Some brief remarks on this definition: We have deliberately suppressed the domain $D$ from the tuple $S$, since for the purposes of this paper, only the ordering induced by $\leq$ on the subsets in $\mathcal{F}$ is relevant. The algebra $\mathcal{F}$ also does not play a significant role in this paper. Unless we say otherwise, we assume $\mathcal{F}$ contains all subsets of interest and suppress mention of $\mathcal{F}$, denoting a plausibility space as a pair $(W, \mathrm{Pl})$.

Clearly plausibility spaces generalize probability spaces. In (Friedman \& Halpern, 1998b, 1995) we show that they also generalize belief function (Shafer, 1976), fuzzy measures (Wang \& Klir, 1992), possibility measures (Dubois \& Prade, 1990), ordinal ranking (or $\kappa$-ranking) (Goldszmidt \& Pearl, 1996; Spohn, 1988), preference orderings (Kraus, Lehmann, \& Magidor, 1990; Shoham, 1987), and parameterized probability distributions (Goldszmidt, Morris, \& Pearl, 1993) that are used as a basis for Pearl's 6 -semantics for defaults (Pearl, 1989).

Our goal is to describe the agent's beliefs in terms of plausibility. To do this, we describe how to evaluate statements of the form $B \varphi$ given a plausibility space. In fact, we use a richer logical language that also allows us to describe how the agent compares different 
alternatives. This is the logic of conditionals. Conditionals are statements of the form $\varphi \rightarrow \psi$, read "given $\varphi, \psi$ is plausible" or "given $\varphi$, then by default $\psi$ ". The syntax of the logic of conditionals is simple: we start with primitive propositions and close off under conjunction, negation and the modal operator $\rightarrow$. The resulting language is denoted $\mathcal{L}^{C}$.

A plausibility structure is a tuple $P L=(W, \mathrm{Pl}, \pi)$, where $W$ is a set of possible worlds, $\mathrm{P} 1$ is a plausibility measure on $W$, and $\pi(w)$ is a truth assignment to primitive propositions. Given a plausibility structure $P L=(W, \mathrm{Pl}, \pi)$, we define $\llbracket \varphi \rrbracket P L=\{w \in W: \pi(w) \models \varphi\}$ to be the set of worlds that satisfy $\varphi$. We omit the subscript $P L$, when it is clear from the context. Conditionals are evaluated according to a rule that is essentially the same as the one used by Dubois and Prade (1991) to evaluate conditionals using possibility measures:

- $P L \models \varphi \rightarrow \psi$ if either $\mathrm{Pl}(\llbracket \varphi \rrbracket)=\perp$ or $\mathrm{Pl}(\llbracket \varphi \wedge \psi \rrbracket)>\mathrm{Pl}(\llbracket \varphi \wedge \neg \psi \rrbracket)$.

Intuitively, $\varphi \rightarrow \psi$ holds vacuously if $\varphi$ is impossible; otherwise, it holds if $\varphi \wedge \psi$ is more plausible than $\varphi \wedge \neg \psi$. As we show in (Friedman \& Halpern, 1998b), this semantics of conditionals also generalizes the semantics of conditionals in $\kappa$-ranking (Goldszmidt \& Pearl, 1996), and PPD structures (Goldszmidt et al., 1993). As we also show in (Friedman \& Halpern, 1998b), this semantics for conditionals generalizes the semantics of preferential structures. As this relationship plays a role in the discussion below, we review the necessary definitions here. A preferential structure is a tuple $(W, \prec, \pi)$, where $\prec$ is a partial order on $W$. Roughly speaking, $w \prec w^{\prime}$ holds if $w$ is preferred to $w^{\prime} .^{3}$ The intuition (Shoham, 1987 ) is that a preferential structure satisfies a conditional $\varphi \rightarrow \psi$ if all the most preferred worlds (i.e., the minimal worlds according to $\prec$ ) in $\llbracket \varphi \rrbracket$ satisfy $\psi$. However, there may be no minimal worlds in $\llbracket \varphi \rrbracket$. This can happen if $\llbracket \varphi \rrbracket$ contains an infinite descending sequence $\ldots \prec w_{2} \prec w_{1}$. What do we do in these structures? There are a number of options: the first is to assume that, for each formula $\varphi$, there are minimal worlds in $\llbracket \varphi \rrbracket$; this is the assumption actually made in (Kraus et al., 1990), where it is called the smoothness assumption. A yet more general definition - one that works even if $\prec$ is not smooth - is given in (Lewis, 1973; Boutilier, 1994a). Roughly speaking, $\varphi \longrightarrow \psi$ is true if, from a certain point on, whenever $\varphi$ is true, so is $\psi$. More formally,

( $W, \prec, \pi$ ) satisfies $\varphi \rightarrow \psi$, if for every world $w_{1} \in \llbracket \varphi \rrbracket$, there is a world $w_{2}$ such that (a) $w_{2} \preceq w_{1}$ (so that $w_{2}$ is at least as normal as $w_{1}$ ), (b) $w_{2} \in \llbracket \varphi \wedge \psi \rrbracket$, and

(c) for all worlds $w_{3} \prec w_{2}$, we have $w_{3} \in \llbracket \varphi \Rightarrow \psi \rrbracket$ (so any world more normal than $w_{2}$ that satisfies $\varphi$ also satisfies $\psi$ ).

It is easy to verify that this definition is equivalent to the earlier one if $\prec$ is smooth.

Proposition 2.2: (Friedman \& Halpern, 1998b) If $\prec$ is a preference ordering on $W$, then there is a plausibility measure $\mathrm{Pl}_{\prec}$ on $W$ such that $(W, \prec, \pi) \models \varphi \rightarrow \psi$ if and only if $\left(W, \mathrm{Pl}_{\prec}, \pi\right) \models \varphi \rightarrow \psi$.

We briefly describe the construction of $\mathrm{P} 1_{\prec}$ here, since we use it in the sequel. Given a preference order $\prec$ on $W$, let $D_{0}$ be the domain of plausibility values consisting of one

3. We follow the standard notation for preference here (Kraus et al., 1990), which uses the (perhaps confusing) convention of placing the more likely (or less abnormal) world on the left of the $\prec$ operator. Unfortunately, when translated to plausibility, this will mean $w \prec w^{\prime}$ holds iff $\operatorname{Pl}\left(\{w\}>\operatorname{Pl}\left(\left\{w^{\prime}\right\}\right)\right.$. 
element $d_{w}$ for every element $w \in W$. We define a partial order on $D_{0}$ using $\prec: d_{v}<d_{w}$ if $w \prec v$. (Recall that $w \prec w^{\prime}$ denotes that $w$ is preferred to $w^{\prime}$.) We then take $D$ to be the smallest set containing $D_{0}$ that is closed under least upper bounds (so that every set of elements in $D$ has a least upper bound in $D$ ). For a subset $A$ of $W$, we can then define $\mathrm{Pl}_{\prec}(A)$ to be the least upper bound of $\left\{d_{w}: w \in A\right\}$. Since $D$ is closed under least upper bounds, $\mathrm{Pl}(A)$ is well defined. As we show in (Friedman \& Halpern, 1998b), this choice of $\mathrm{P} l_{\prec}$ satisfies Proposition 2.2.

The results of (Friedman \& Halpern, 1998b) show that this semantics for conditionals generalizes previous semantics for conditionals. Does this semantics capture our intuitions about conditionals? In the AI literature, there has been little consensus on the "right" properties for defaults (which are essentially conditionals). However, there has been some consensus on a reasonable "core" of inference rules for default reasoning. This core is usually known as the KLM properties (Kraus et al., 1990), and includes such properties as

AND From $\varphi \rightarrow \psi_{1}$ and $\varphi \rightarrow \psi_{2}$ infer $\varphi \rightarrow \psi_{1} \wedge \psi_{2}$

OR From $\varphi_{1} \rightarrow \psi$ and $\varphi_{2} \rightarrow \psi$ infer $\varphi_{1} \vee \varphi_{2} \rightarrow \psi$

What constraints on plausibility spaces gives us the KLM properties? Consider the following two conditions:

A2 If $A, B$, and $C$ are pairwise disjoint sets, $\mathrm{Pl}(A \cup B)>\mathrm{Pl}(C)$, and $\mathrm{Pl}(A \cup C)>$ $\mathrm{Pl}(B)$, then $\mathrm{Pl}(A)>\mathrm{Pl}(B \cup C)$.

A3 If $\operatorname{Pl}(A)=\operatorname{Pl}(B)=\perp$, then $\operatorname{Pl}(A \cup B)=\perp$.

A plausibility space $(W, \mathrm{P} 1)$ is qualitative if it satisfies $\mathrm{A} 2$ and A3. A plausibility structure $(W, \mathrm{Pl}, \pi)$ is qualitative if $(W, \mathrm{Pl})$ is a qualitative plausibility space. In (Friedman \& Halpern, 1998b), we show that, in a very general sense, qualitative plausibility structures capture default reasoning. More precisely, we show that the KLM properties are sound with respect to a class of plausibility structures if and only if the class consists of qualitative plausibility structures. (We also provide a weak condition that we show is necessary and sufficient for the KLM properties to be complete.) These results show that plausibility structures provide a unifying framework for the characterization of default entailment in these different logics.

\subsection{Plausibility and Knowledge}

In (Friedman \& Halpern, 1997) we show how plausibility measures can be incorporated into the multi-agent system framework of (Halpern \& Fagin, 1989). This allows us to describe the agent's assessment of the possible states the system is in at each point in time. At the same time we also introduce conditionals into the logical language in order to reason about these plausibility assessments. We now review the relevant details.

An (interpreted) plausibility system is a tuple $(\mathcal{R}, \pi, \mathcal{P})$ where, as before, $\mathcal{R}$ is a set of runs and $\pi$ maps each point to a truth assignment, and where $\mathcal{P}$ is a plausibility assignment function mapping each point $(r, m)$ to a qualitative plausibility space $\mathcal{P}(r, m)=$ $\left(W_{(r, m)}, \mathrm{Pl} l_{(r, m)}\right)$. Intuitively, the plausibility space $\mathcal{P}(r, m)$ describes the relative plausibility of events from the point of view of the agent at $(r, m)$. In this paper, we restrict our attention to plausibility spaces that satisfy two additional assumptions: 
- $W_{(r, m)}=\left\{\left(r^{\prime}, m^{\prime}\right) \mid(r, m) \sim_{a}\left(r^{\prime}, m^{\prime}\right)\right\}$. Thus, the agent considers plausible only situations that are possible according to her knowledge.

- if $(r, m) \sim_{a}\left(r^{\prime}, m^{\prime}\right)$ then $\mathcal{P}(r, m)=\mathcal{P}\left(r^{\prime}, m^{\prime}\right)$. This means that the plausibility space is a function of the agent's local state. ${ }^{4}$

We define a logical language to reason about interpreted systems. The syntax of the logic is simple; we start with primitive propositions and close off under conjunction, negation, the $K$ modal operator ( $K \varphi$ says that the agent knows $\varphi$ ), the $\bigcirc$ modal operator $(\bigcirc \varphi$ says that $\varphi$ is true at the next time step), and the $\rightarrow$ modal operator. The resulting language is denoted $\mathcal{L}^{K P T}{ }^{5}$ We recursively assign truth values to formulas in $\mathcal{L}^{K P T}$ at a point $(r, m)$ in a plausibility system $\mathcal{I}$. The truth of primitive propositions is determined by $\pi$, so that

$$
(\mathcal{I}, r, m) \models p \text { if } \pi(r, m)(p)=\text { true. }
$$

Conjunction and negation are treated in the standard way, as is knowledge: The agent knows $\varphi$ at $(r, m)$ if $\varphi$ holds at all points that she cannot distinguish from $(r, m)$. Thus,

$$
(\mathcal{I}, r, m) \models K \varphi \text { if }\left(\mathcal{I}, r^{\prime}, m^{\prime}\right) \models \varphi \text { for all }\left(r^{\prime}, m^{\prime}\right) \sim_{a}(r, m) .
$$

O $\varphi$ is true at $(r, m)$ if $\varphi$ is true at $(r, m+1)$. Thus,

$$
(\mathcal{I}, r, m) \models \bigcirc \varphi \text { if }(\mathcal{I}, r, m+1) \models \varphi \text {. }
$$

Finally, we define the conditional operator $\rightarrow$ to describe the agent's plausibility assessment at the current time. Let $\llbracket \varphi \rrbracket_{(r, m)}=\left\{\left(r^{\prime}, m^{\prime}\right) \in W_{(r, m)}:(\mathcal{I}, r, m) \models \varphi\right\}$.

$(\mathcal{I}, r, m) \models \varphi \rightarrow \psi$ if either $\mathrm{Pl}_{(r, m)}\left(\llbracket \varphi \rrbracket_{(r, m)}\right)=\perp$ or $\mathrm{P}_{(r, m)}\left(\llbracket \varphi \wedge \psi \rrbracket_{(r, m)}\right)>\mathrm{Pl}_{(r, m)}\left(\llbracket \varphi \wedge \neg \psi \rrbracket_{(r, m)}\right)$.

We now define a notion of belief. Intuitively, the agent believes $\varphi$ if $\varphi$ is more plausible than not. Formally, we define $B \varphi \Leftrightarrow($ true $\rightarrow \varphi)$.

In (Friedman \& Halpern, 1997) we prove that, in this framework, knowledge is an S5 operator, the conditional operator $\rightarrow$ satisfies the usual axioms of conditional logic (Burgess, 1981), and $\bigcirc$ satisfies the usual properties of temporal logic (Manna \& Pnueli, 1992). In addition, these properties imply that belief is a K45 operator, and the interactions between knowledge and belief are captured by the axioms $K \varphi \Rightarrow B \varphi$ and $B \varphi \Rightarrow K B \varphi$.

Example 2.3: (Friedman \& Halpern, 1997) We add a plausibility measure to the system defined in Example 2.1. We define $\mathcal{I}_{\text {diag }}=\left(\mathcal{R}_{\text {diag }}, \pi_{\text {diag }}, \mathcal{P}_{\text {diag }}\right)$, where $\mathcal{P}_{\text {diag }}$ is the plausibility assignment we now describe. We assume that failures of individual components are independent of one another. If we also assume that the likelihood of each component failing is the same, and also that this likelihood is small (i.e., failures are exceptional), then we can construct a plausibility measure as follows. If $\left(r^{\prime}, m\right)$ and $\left(r^{\prime \prime}, m\right)$ are two points in $W_{(r, m)}$, we say that $\left(r^{\prime}, m\right)$ is more plausible than $\left(r^{\prime \prime}, m\right)$ if $\mid$ fault $\left(r^{\prime}, m\right)|<|$ fault $\left(r^{\prime \prime}, m\right) \mid$,

4. The framework presented in (Friedman \& Halpern, 1997) is more general than this, dealing with multiple agents and allowing the agent to consider several plausibility spaces in each local state. The simplified version we present here suffices to capture belief revision and update.

5. It is easy to add other temporal modalities such as until, eventually, since, etc. These do not play a role in this paper. 
that is, if the failure set at $\left(r^{\prime}, m\right)$ consists of fewer faulty components than at $\left(r^{\prime \prime}, m\right)$. We extend these comparisons to sets: $\mathrm{Pl}_{(r, m)}(A) \leq \mathrm{Pl}_{(r, m)}(B)$ if $\min _{\left(r^{\prime}, m\right) \in A}\left(\mid\right.$ fault $\left.\left(r^{\prime}, m\right) \mid\right) \geq$ $\min _{\left(r^{\prime}, m\right) \in B}\left(\mid\right.$ fault $\left.\left(r^{\prime}, m\right) \mid\right)$; that is, $A$ is less plausible if all the points in $A$ have failure sets of larger cardinality then the minimal one in $B$. With this plausibility measure, if all of the agent's observations up to time $m$ are consistent with there being no failures, then the agent believes that all components are functioning correctly. On the other hand, if the observations do not match the expected output of the circuit, then the agent considers minimal failure sets that are consistent with her observations. Thus, if the observations are consistent with a failure of $c_{1}$, or a failure of $c_{3}$, or the combined failure of $c_{2}$ and $c_{7}$, then the agent believes that either $c_{1}$ or $c_{3}$ is faulty, but not both.

We now make this more precise. A failure set (i.e., a diagnosis) is characterized by a complete formula over $f_{1}, \ldots, f_{n}$ - that is, one that determines the truth values all these propositions. For example, if $n=3$, then $f_{1} \wedge \neg f_{2} \wedge \neg f_{3}$ characterizes the failure set $\left\{c_{1}\right\}$. We define $D_{(r, m)}$ to be the set of failure sets (i.e., diagnoses) that the agent considers possible at $(r, m)$; that is $D_{(r, m)}=\left\{f \in F:\left(\mathcal{I}_{\text {diag }}, r, m\right) \models \neg B \neg f\right\}$ where $F$ is the set of all possible failure sets.

Belief change in $\mathcal{I}_{\text {diag }}$ is characterized by the following proposition.

Proposition 2.4: If there is some $f \in D_{(r, m)}$ that is consistent with the new observation $i o(r, m+1)$, then $D_{(r, m+1)}$ consists of all the failure sets in $D_{(r, m)}$ that are consistent with $i o(r, m+1)$. If all $f \in D_{(r, m)}$ are inconsistent with io $(r, m+1)$, then $D_{(r, m+1)}$ consists of all failure sets of cardinality $j$ that are consistent with io $(r, 1), \ldots$, io $(r, m+1)$, where $j$ is the least cardinality for which there is at least one failure set consistent with these observations.

Thus, in $\mathcal{I}_{\text {diag }}$, a new observation consistent with the current set of most likely explanations reduces this set (to those consistent with the new observation). On the other hand, a surprising observation (one inconsistent with the current set of most likely explanations) has a rather drastic effect. It easily follows from Proposition 2.4 that if $i o(r, m+1)$ is surprising, then $D_{(r, m)} \cap D_{(r, m+1)}=\emptyset$, so the agent discards all her current explanations in this case. Moreover, an easy induction on $m$ shows that if $D_{(r, m)} \cap D_{(r, m+1)}=\emptyset$, then the cardinality of the failure sets in $D_{(r, m+1)}$ is greater than the cardinality of failure sets in $D_{(r, m)}$. Thus, in this case, the explanations in $D_{(r, m+1)}$ are more complicated than those in $D_{(r, m)}$.

\subsection{Conditioning}

In an interpreted system, the agent's beliefs change from point to point as her plausibility space changes. The general framework does not put any constraints on how the plausibility space changes. If we were thinking probabilistically, we could imagine the agent starting with a prior on the runs in the system. Since a run describes a complete history over time, this means that the agent puts a prior probability on the possible sequences of events that could happen. We would then expect the agent to modify her prior by conditioning on whatever information she has learned. As we show below, this notion of conditioning is closely related to belief revision and update. We remark that we are not the first to applying conditioning in the context of belief change (cf. (Goldszmidt \& Pearl, 1996; Spohn, 1988)); the details are a little more complex in our framework, because we model time explicitly. 
We start by making the simplifying assumption that we are dealing with synchronous systems where agents have perfect recall (Halpern \& Vardi, 1989). Intuitively, this means that the agent knows what the time is and does not forget the observations she has made. Formally, a system is synchronous if $(r, m) \sim_{a}\left(r^{\prime}, m^{\prime}\right)$ only if $m=m^{\prime}$. In synchronous systems, the agent has perfect recall if $\left(r^{\prime}, m+1\right) \sim_{a}(r, m+1)$ implies $\left(r^{\prime}, m\right) \sim_{a}(r, m)$. Thus, the agent considers run $r$ possible at the point $(r, m+1)$ only if she also considers it possible at $(r, m)$. This means that any runs considered impossible at $(r, m)$ are also considered impossible at $(r, m+1)$ : the agent does not forget what she knew.

Just as with probability, we assume that the agent has a prior plausibility measure on runs that describes her prior assessment on the possible executions of the system. As the agent gains knowledge, she updates her prior by conditioning. More precisely, at each point $(r, m)$, the agent conditions her previous assessment on the set of runs considered possible at $(r, m)$. This results in an updated assessment (posterior) of the plausibility of runs. This posterior induces, via a projection from runs to points, a plausibility measure on points. We can think of the agent's posterior at time $m$ as simply her prior conditioned on her knowledge at time $m$.

Formally, the prior plausibility of the agent is a plausibility measure $\mathcal{P}_{a}=\left(\mathcal{R}, \mathrm{Pl}_{a}\right)$ over the runs in the system. If $A$ is a set of points, we define $\mathcal{R}(A)=\{r: \exists m((r, m) \in A)\}$ to be the set of runs on which the points in $A$ lie. The agent updates plausibilities by conditioning in $\mathcal{I}$ if the following condition is met:

PRIOR There is prior $\mathcal{P}_{a}=\left(\mathcal{R}, \mathrm{P} l_{a}\right)$ such that for all runs $r \in \mathcal{R}$, times $m$, and sets $A, B \subseteq W_{(r, m)}, \mathrm{Pl}_{(r, m)}(A) \leq \mathrm{Pl}_{(r, m)}(B)$ if and only if $\mathrm{Pl}_{a}(\mathcal{R}(A)) \leq$ $\mathrm{Pl}_{a}(\mathcal{R}(B))$.

This definition implies that the agent's plausibility assessment at each point is determined, in a straightforward fashion, by her prior.

As shown in (Friedman \& Halpern, 1997), in synchronous systems that satisfy PRIOR where agent have perfect recall, we can say even more: the agent's plausibility measure at time $m+1$ is determined by her plausibility measure at time $m$. To make this precise, if $A$ is a set of points, let $\operatorname{prev}(A)=\{(r, m):(r, m+1) \in A\}$.

Theorem 2.5: (Friedman \& Halpern, 1997). Let $\mathcal{I}$ be a synchronous system satisfying PRIOR where agents have perfect recall. Then $\mathrm{P} l_{(r, m+1)}(A) \leq \mathrm{Pl}_{(r, m+1)}(B)$ if and only if $\mathrm{Pl}_{(r, m)}(\operatorname{prev}(A)) \leq \mathrm{Pl}_{(r, m)}(\operatorname{prev}(B))$, for all runs $r$, times $m$, and sets $A, B \subseteq W_{(r, m+1)}$.

Thus, in synchronous systems where agents have perfect recall PRIOR implies a "local" rule for update that incrementally changes the agent's plausibility at each step. This local rule consists of two steps. First, the agent's plausibility at time $m$ is projected to time $m+1$ points. Second, time $m+1$ points that are inconsistent with the agent knowledge at $(r, m+1)$ are discarded. This procedure implies that the relative plausibility of two sets of runs does not change unless one of them is incompatible with the new knowledge.

Example 2.6: It is easy to verify that the system $\mathcal{I}_{\text {diag }}$ we consider in Example 2.3 satisfies PRIOR. The prior $\mathcal{P}_{a}$ is determined by the failure set in each run in a manner similar to the construction of $\mathrm{Pl}_{(r, m)}$. That is, $R_{1}$ is more plausible than $R_{2}$ if there is a run in $R_{1}$ with a smaller failure set than all the runs in $R_{2}$. 


\section{Review of Revision and Update}

We now present a brief review of belief revision and update.

Belief revision attempts to describe how a rational agent incorporates new beliefs. As we said earlier, the main intuition is that as few changes as possible should be made. Thus, when something is learned that is consistent with earlier beliefs, it is just added to the set of beliefs. The more interesting situation is when the agent learns something inconsistent with her current beliefs. She must then discard some of her old beliefs in order to incorporate the new belief and remain consistent. The question is which ones?

The most widely accepted notion of belief revision is defined by the AGM theory (A1chourrón et al., 1985; Gärdenfors, 1988). This theory was originally developed in philosophy of science, where one attempts to understand when a scientist changes her beliefs (e.g., theory of physical laws) in a rational manner. In this context, it seems reasonable to assume that the world is static; that is, the laws of physics do not change while the scientist is performing experiments.

Formally, this theory assumes a logical language $\mathcal{L}_{e}$ over a set $\Phi_{e}$ of primitive propositions with a consequence relation $\vdash_{\mathcal{L}_{e}}$ that contains the propositional calculus and satisfies the deduction theorem. The AGM approach assumes that an agent's epistemic state is represented by a belief set, that is, a set $K$ of formulas in the language $\mathcal{L}_{e}{ }^{6}{ }^{6}$ There is also assumed to be a revision operator $o$ that takes a belief set $A$ and a formula $\varphi$ and returns a new belief set $A \circ \varphi$, intuitively, the result of revising $A$ by $\varphi$. The following AGM postulates are an attempt to characterize the intuition of "minimal change":

(R1) $A \circ \varphi$ is a belief set

(R2) $\varphi \in A \circ \varphi$

(R3) $A \circ \varphi \subseteq C l(A \cup\{\varphi\})^{7}$

(R4) If $\neg \varphi \notin A$ then $C l(A \cup\{\varphi\}) \subseteq A \circ \varphi$

(R5) $A \circ \varphi=C l$ (false) if and only if $\vdash_{\mathcal{L}_{e}} \neg \varphi$

(R6) If $\vdash_{\mathcal{L}_{e}} \varphi \Leftrightarrow \psi$ then $A \circ \varphi=A \circ \psi$

(R7) $A \circ(\varphi \wedge \psi) \subseteq C l(A \circ \varphi \cup\{\psi\})$

(R8) If $\neg \psi \notin A \circ \varphi$ then $C l(A \circ \varphi \cup\{\psi\}) \subseteq A \circ(\varphi \wedge \psi)$.

The essence of these postulates is the following. After a revision by $\varphi$ the belief set should include $\varphi$ (postulates R1 and R2). If the new belief is consistent with the belief set, then the revision should not remove any of the old beliefs and should not add any new beliefs except these implied by the combination of the old beliefs with the new belief (postulates R3 and R4). This condition is called persistence. The next two conditions discuss the coherence of beliefs. Postulate R5 states that the agent is capable of incorporating any consistent belief and postulate R6 states that the syntactic form of the new belief does not affect the revision process. The last two postulates enforce a certain coherency on the

6. For example, Gärdenfors (1988, p. 21) says "A simple way of modeling the epistemic state of an individual is to represent it by a set of sentences."

7. $C l(A)=\left\{\varphi \mid A \vdash_{\mathcal{L}_{e}} \varphi\right\}$ is the deductive closure of a set of formulas $A$. 
outcome of revisions by related beliefs. Basically, they state that if $\psi$ is consistent with $A \circ \varphi$ then $A \circ(\varphi \wedge \psi)$ is just $A \circ \varphi \circ \psi$.

The notion of belief update originated in the database community (Keller \& Winslett, 1985; Winslett, 1988). The problem is how a knowledge base should change when something is learned about the world. For example, suppose that a transaction adds to the knowledge base the fact "Table 7 is in Office 2", which contradicts the previous belief that "Table 7 is in Office 1". What else should change? The intuition that update attempts to capture is that such a transaction describes a change that has occurred in the world. Thus, in our example, by applying update we might conclude that the reason that the table is in Office 2 is that it was moved, not that our earlier beliefs were false. This example shows that, unlike revision, update does not assume that the world is static.

Katsuno and Mendelzon (1991a) suggest a set of postulates that an update operator should satisfy. The update postulates are expressed in terms of formulas, not belief sets. That is, an update operator $\diamond$ maps a pair of formulas, one describing the agent's current beliefs and the other describing the new observation, to a new formula that describes the agent's updated beliefs. This is not unreasonable, since we can identify a formula $\varphi$ with the belief set $C l(\varphi)$. Indeed, if $\Phi$ is finite (which is what Katsuno and Mendelzon assume) every belief set $A$ can be associated with some formula $\varphi_{A}$ such that $C l\left(\varphi_{A}\right)=A$, and every formula $\varphi$ corresponds to a belief set $C l(\varphi)$. Thus, any update operator induces an operator that maps a belief state and an observation to a new belief state. We slightly abuse notation and use the same symbol to denote both types of mappings. We say that a belief set $A$ is complete if, for every $\varphi \in \mathcal{L}_{e}$, either $\varphi \in A$ or $\neg \varphi \in A$. A formula $\mu$ is complete if $\mathrm{Cl}(\mu)$ is complete.

The KM postulates are:

(U1) $\vdash_{\mathcal{L}_{e}} \mu \diamond \varphi \Rightarrow \varphi$

(U2) If $\vdash_{\mathcal{L}_{e}} \mu \Rightarrow \varphi$, then $\vdash_{\mathcal{L}_{e}} \mu \diamond \varphi \Leftrightarrow \mu$

(U3) $\vdash_{\mathcal{L}_{e}} \neg \mu \diamond \varphi$ if and only if $\vdash_{\mathcal{L}_{e}} \neg \mu$ or $\vdash_{\mathcal{L}_{e}} \neg \varphi$

(U4) If $\vdash_{\mathcal{L}_{e}} \mu_{1} \Leftrightarrow \mu_{2}$ and $\vdash_{\mathcal{L}_{e}} \varphi_{1} \Leftrightarrow \varphi_{2}$ then $\vdash_{\mathcal{L}_{e}} \mu_{1} \diamond \varphi_{1} \Leftrightarrow \mu_{2} \diamond \varphi_{2}$

(U5) $\vdash_{\mathcal{L}_{e}}(\mu \diamond \varphi) \wedge \psi \Rightarrow \mu \diamond(\varphi \wedge \psi)$

(U6) If $\vdash_{\mathcal{L}_{e}} \mu \diamond \varphi_{1} \Rightarrow \varphi_{2}$ and $\vdash_{\mathcal{L}_{e}} \mu \diamond \varphi_{2} \Rightarrow \varphi_{1}$, then $\vdash_{\mathcal{L}_{e}} \mu \diamond \varphi_{1} \Leftrightarrow \mu \diamond \varphi_{2}$

(U7) If $\mu$ is complete then $\vdash_{\mathcal{L}_{e}}\left(\mu \diamond \varphi_{1}\right) \wedge\left(\mu \diamond \varphi_{2}\right) \Rightarrow \mu \diamond\left(\varphi_{1} \vee \varphi_{2}\right)$

(U8) $\vdash_{\mathcal{L}_{e}}\left(\mu_{1} \vee \mu_{2}\right) \diamond \varphi \Leftrightarrow\left(\mu_{1} \diamond \varphi\right) \vee\left(\mu_{2} \diamond \varphi\right)$.

The essence of these postulates is as following. After learning $\varphi$, the agent believes $\varphi$ (postulate $\mathrm{U} 1$, which is analogous to R2). If $\varphi$ is already believed, then updating by $\varphi$ does not change the agent's beliefs (postulate U2, which is a weaker version of R3 and R4). The next two postulates (U3 and U4) deal with coherence of the belief change process. They are analogous to R5 and R6, respectively, with minor differences. Postulates U5 and U6 deal with observations that are related to each other. U5 states that beliefs after learning $\varphi$ that are consistent with $\psi$ are also believed after learning $\varphi \wedge \psi$. U6 states that if $\varphi_{2}$ is believed after learning $\varphi_{1}$ and $\varphi_{1}$ is believed after learning $\varphi_{2}$, then learning either $\varphi_{1}$ or $\varphi_{2}$ leads to the same belief set. Finally, U7 and U8 deal with decomposition properties of the update operation. U7 states that if $\mu$ is essentially a truth assignment to $\mathcal{L}$, then if $\psi$ 
is believed after learning $\varphi_{1}$ and is also believed after learning $\varphi_{2}$ then it is believed after learning $\varphi_{1} \vee \varphi_{2}$. U8 states that the update of the knowledge base can be computed by independent updates on each sub-part of the knowledge. That is, if $\mu=\mu_{1} \vee \mu_{2}$, then we can apply update to each of $\mu_{1}$ and $\mu_{2}$, and then combine the results.

\section{Belief Change Systems}

We want to model belief change-particularly belief revision and belief update-in the framework of systems. To do so, we consider a particular class of systems that we call belief change systems. In belief change systems, the agent makes observations about an external environment. Just as is (implicitly) assumed in both revision and update, we assume that these observations are described by formulas in some logical language. We then make other assumptions regarding the plausibility measure used by the agent. We formalize our assumptions as conditions BCS1-BCS5, described below, and say that a system $\mathcal{I}=(\mathcal{R}, \pi, \mathcal{P})$ is a belief change system if it satisfies these conditions. We denote by $\mathcal{C}^{B C S}$ the set of belief change systems.

Assumption BCS1 formalizes the intuition that our language includes propositions for reasoning about the environment, whose truth depends only on the environment state.

BCS1 The language $\mathcal{L}$ includes a propositional sublanguage $\mathcal{L}_{e}$ over a set $\Phi_{e}$ of primitive propositions. $\mathcal{L}_{e}$ contains the usual propositional connectives and comes equipped with a consequence relation $\vdash_{\mathcal{L}_{e}}$. The interpretation $\pi(r, m)$ assigns truth to propositions in $\Phi_{e}$ in such a way that

(a) $\pi(r, m)$ is consistent with $\vdash_{\mathcal{L}_{e}}$, that is, $\left\{p: p \in \Phi_{e}, \pi(r, m)(p)=\right.$ true $\} \cup$ $\left\{\neg p: p \in \Phi_{e}, \pi(r, m)(p)=\right.$ false $\}$ is $\vdash_{\mathcal{L}_{e}}$ consistent, and

(b) $\pi(r, m)(p)$ depends only on $r_{e}(m)$ for propositions in $\Phi_{e}$; that is, $\pi(r, m)(p)=$ $\pi\left(r^{\prime}, m^{\prime}\right)(p)$ whenever $r_{e}(m)=r_{e}^{\prime}\left(m^{\prime}\right)$.

Part (b) of BCS1 implies that we can evaluate formulas in $\mathcal{L}_{e}$ with respect to environment states; that is, if $\varphi \in \mathcal{L}_{e}$ and $r_{e}(m)=r_{e}^{\prime}\left(m^{\prime}\right)$, then $(\mathcal{I}, r, m) \models \varphi$ if and only if $\left(\mathcal{I}, r^{\prime}, m^{\prime}\right) \models \varphi$. Since the environment is all that is relevant for formulas in $\mathcal{L}_{e}$, if $\varphi \in \mathcal{L}_{e}$, we write $s_{e} \vDash \varphi$ if $(\mathcal{I}, r, m) \models \varphi$ for some point $(r, m)$ such that $r_{e}(m)=s_{e}$.

BCS2 is concerned with the form of the agent's local state. Recall that, in our framework, the local state captures the relevant aspects of the agent's epistemic state. The functional form of the revision and update operators suggests that all that matters regarding how an agent changes her beliefs are the agent's current epistemic state (which is taken by both AGM and KM to be a belief set) and what is learned. In terms of our framework, this suggests that agent's local state at time $m+1$ should be a function of her local state of time $m$ and the observation made at time $m$. We in fact make the stronger assumption here that the agent's state consists of the sequence of observations made by the agent. This means that the agent remembers all her past observations. Note that this surely implies that the agent's local state at time $m+1$ is determined by her state at time $m$ and the observation made at time $m$. We make the further assumption that the observations made by the agent can be described by formulas in $\mathcal{L}_{e}$. Although this is quite a strong assumption on the expressive power of $\mathcal{L}_{e}$, it is standard in the literature: both revision and update 
assume that observations can be expressed as formulas in the language (see Section 3 ). These assumptions are formalized in BCS2:

BCS2 For all $r \in R$ and for all $m$, we have $r_{a}(m)=\left\langle o_{(r, 1)}, \ldots, o_{(r, m)}\right\rangle$ where $o_{(r, k)} \in \mathcal{L}_{e}$ for $1 \leq k \leq m$.

Intuitively, $o_{(r, k)}$ is the observation the agent makes immediately after the transition from time $k \Leftrightarrow 1$ to time $k$ in run $r$. Thus, it represents what the agent observes about the new state of the system at time $k$. Note that BCS2 implies that the agent's state at time 0 is the empty sequence in all runs. Moreover, it implies that $r_{a}(m+1)=r_{a}(m) \cdot o_{(r, m+1)}$, where $\cdot$ is the append operation on sequences. That is, the agent's state at $(r, m+1)$ is the result of appending to her previous state the latest observation she has made about the system. It is not too hard to show that belief change systems are synchronous and agents in them have perfect recall. (We remark that the agents' local states are modeled in a similar way in the model of knowledge bases presented in (Fagin et al., 1995).)

Clearly we want to reason in our language about the observations the agent makes. Thus, we assume that the language includes propositions that describe the observations made by the agent.

BCS3 The language $\mathcal{L}$ includes a set $\Phi_{o b s}$ of primitive propositions disjoint from $\Phi_{e}$ such that $\Phi_{o b s}=\left\{\operatorname{learn}(\varphi): \varphi \in \mathcal{L}_{e}\right\}$. Moreover, $\pi(r, m)(\operatorname{learn}(\varphi))=$ true if and only if $o_{(r, m)}=\varphi$ for all runs $r$ and times $m$.

In a system satisfying BCS1-BCS3, we can talk about belief change. The agent's state encodes observations, and we have propositions that allow us to talk about what is observed. The next assumption is somewhat more geared to situations where observations are always "accepted", so that after the agent observes $\varphi$, she believes $\varphi$. While this is not a necessary assumption, it is made by both belief revision and belief update. We capture this assumption here in what is perhaps the simplest possible way: by assuming that observations are reliable, so that the agent observes $\varphi$ only if the current state of the environment satisfies $\varphi$. This is certainly not the only way of enforcing the assumption that observations are accepted, but it is perhaps the simplest, so we focus on it here. As we shall see, this assumption is consistent with both revision and update, in the sense that we can capture both in systems satisfying it.

BCS4 $(\mathcal{I}, r, m) \models o_{(r, m)}$ for all runs $r$ and times $m$.

Note that BCS4 implies that the agent never observes false. Moreover, it implies that after observing $\varphi$, the agent knows that $\varphi$ is true. In (Boutilier et al., 1998), we consider an instance of our framework in which observations are unreliable (so that BCS4 does not hold in general), and examine the status of $\mathrm{R} 2$, the acceptance postulate, in this case.

Finally, we assume that belief change proceeds by conditioning. While there are certainly other assumptions that can be made, as we have tried to argue, conditioning is a principled approach that captures the intuitions of minimal change, given the observations. And, as we shall see, conditioning (as captured by PRIOR) is consistent with both revision and update.

BCS5 $\mathcal{I}$ satisfies PRIOR. 
Many interesting systems can be viewed as BCS's.

Example 4.1: Consider the systems $\mathcal{I}_{\text {diag }, 1}$ and $\mathcal{I}_{\text {diag, } 2}$ of Example 2.1. Are these systems BCSs? Not quite, since $\pi_{\text {diag }}$ is not defined on primitive propositions of the form $\operatorname{learn}(\varphi)$, but we can easily embed both systems in a BCS. Let $\mathcal{L}_{\text {diag }}$ the propositional language defined over $\Phi_{\text {diag }}$, and let $\Phi_{\text {diag }}^{+}$consist of $\Phi_{\text {diag }}$ together with all the primitive propositions of the form learn $(\varphi)$ for $\varphi \in \mathcal{L}_{\text {diag }}$. Let $\pi_{\text {diag }}^{+}$be the obvious extension of $\pi_{\text {diag }}$ to $\Phi_{\text {diag }}^{+}$, defined so that BCS3 holds. Then in it is easy to see that $\left(\mathcal{R}_{\text {diag }}, \pi_{\text {diag }}^{+}, \mathcal{P}_{\text {diag }, i}\right)$ is a BCS: we take the $\Phi_{e}$ of BCS1 to be $\Phi_{\text {diag }}$, and define $\vdash_{\mathcal{L}_{\text {diag }}}$ so that it enforces the relationships determined by the circuit layout. Thus, for example, if $c_{1}$ is an AND gate with input lines $l_{1}$ and $l_{2}$ and output line $l_{3}$, then we would have $\vdash_{\mathcal{L}_{\text {diag }}} \neg f_{1} \Rightarrow\left(h_{3} \Leftrightarrow h_{1} \wedge h_{2}\right)$. It is then easy to see that BCS2-BCS5 hold by our construction.

These definitions set the background for our presentation of belief revision and belief update.

\section{Capturing Revision}

Revision can be captured by restricting to BCSs that satisfy several additional assumptions. Before describing these assumptions, we briefly review a well-known representation of revision that will help motivate them.

While there are several representation theorems for belief revision, the clearest is perhaps the following (Grove, 1988; Katsuno \& Mendelzon, 1991b). We associate with each belief set $A$ a set $W_{A}$ of possible worlds that consists of those worlds where $A$ is true. Thus, an agent whose belief set is $A$ believes that one of the worlds in $W_{A}$ is the real world. An agent that performs belief revision behaves as though in each belief state $A$ she has a ranking, i.e., a total preorder, over all possible worlds such that the minimal (i.e., most plausible) worlds in the ranking are exactly those in $W_{A}$. When revising by $\varphi$, the agent chooses the minimal worlds satisfying $\varphi$ in the ranking and constructs a belief set from them. It is easy to see that this procedure for belief revision satisfies the AGM postulates. Moreover, in (Grove, 1988; Katsuno \& Mendelzon, 1991b), it is shown that any belief revision operator can be described in terms of such a ranking.

This representation suggests how we can capture belief revision in our framework. We define $\mathcal{C}^{R} \subseteq \mathcal{C}^{B C S}$ to be the set of belief change systems $\mathcal{I}=(\mathcal{R}, \pi, \mathcal{P})$ that satisfy the conditions REV1-REV4 that we define below.

Revision assumes that the world does not change during the revision process. Formally this implies that propositions in $\Phi_{e}$ do not change their truth value along a run, i.e., $(\mathcal{I}, r, m) \models p$ if and only if $(\mathcal{I}, r, m+1) \models p$ for all $p \in \Phi_{e}$. This says that the state of the world is the same with respect to the properties that the agent reasons about (i.e., the propositions in $\Phi_{e}$ ).

$$
\text { REV1 } \pi(r, m)(p)=\pi(r, 0)(p) \text { for all } p \in \Phi_{e} \text { and points }(r, m) .
$$

Note that REV1 does not necessarily imply that $r_{e}(m)=r_{e}(m+1)$. That is, REV1 allows for a changing environment. The only restriction is that the truth value of propositions that describe the environment does not change. We return to this issue in Section 7. 
The representation of (Grove, 1988; Katsuno \& Mendelzon, 1991a) requires the agent to totally order possible worlds. We put a similar requirement on the agent's plausibility assessment. Recall that BCS5 says that the agent's plausibility is induced by a prior $\mathrm{Pl}_{a}$; REV2 strengthens this assumption.

REV2 The prior $\mathrm{Pl}_{a}$ of BCS5 is ranked; that is, for all $A, B \subseteq \mathcal{R}$, either $\mathrm{Pl}_{a}(A) \leq \mathrm{Pl}_{a}(B)$ or $\mathrm{Pl}_{a}(B) \leq \mathrm{Pl}_{a}(A)$, and $\mathrm{Pl}(A \cup B)=\max (\mathrm{Pl}(A), \mathrm{Pl}(B))$.

The representation of (Grove, 1988; Katsuno \& Mendelzon, 1991a) also requires that the agent considers all truth assignments possible. We need a similar condition, except that we want not only that all truth assignments be considered possible, but that they have nontrivial plausibility (i.e., are more plausible than $\perp$ ) as well.

To make this precise, it is helpful to introduce some notation that will be useful for our later definitions as well. Given a system $\mathcal{I}$ and two sequences $\varphi_{1}, \ldots, \varphi_{k}$ and $o_{1}, \ldots, o_{k^{\prime}}$ of formulas in $\mathcal{L}_{e}$, let $\mathcal{R}\left[\varphi_{1}, \ldots, \varphi_{k} ; o_{1}, \ldots, o_{k^{\prime}}\right]$ consist of all runs $r$ where for each $i$ with $1 \leq i \leq k$, the formula $\varphi_{i}$ is true at $(r, i)$ and the agent observes $o_{1}, \ldots, o_{k^{\prime}}$. That is, $\mathcal{R}\left[\varphi_{0}, \ldots, \varphi_{k} ; o_{1}, \ldots, o_{k^{\prime}}\right]=\left\{r \in \mathcal{I}:(\mathcal{I}, r, i)=\varphi_{i}, i=0, \ldots, k\right.$, and $\left.r_{a}\left(k^{\prime}\right)=\left\langle o_{1}, \ldots, o_{k^{\prime}}\right\rangle\right\}$. We allow either sequence of formulas to be empty, so, for example, $\mathcal{R}[\varphi ; \cdot]$ consists of all runs for which $\varphi$ is true at the initial state. (Note that if REV1 holds, this means that $\varphi$ is true in all subsequent states as well.) We use the notation $\mathcal{R}\left[\varphi_{1}, \ldots, \varphi_{m}\right]$ as an abbreviation for $\mathcal{R}\left[\varphi_{1}, \ldots, \varphi_{m} ; \cdot\right]$.

REV3 If $\varphi \in \mathcal{L}_{e}$ is consistent, then $\mathrm{Pl}_{a}(\mathcal{R}[\varphi])>\perp$.

It might seem that REV1-REV3 capture all of the assumptions made by the representation of (Grove, 1988; Katsuno \& Mendelzon, 1991a). However, there is another assumption implicit in the way revision is performed in these representations that we must make explicit in our representation, because of the way we have distinguished observing $\varphi$ (captured by the formula $\operatorname{learn}(\varphi))$ from $\varphi$ itself. Intuitively, when the agent observes $\varphi$, she updates her plausibility assessment by conditioning on $\varphi$. This is essentially what we can think of the earlier representations as doing. However, in our representation, the agent does not condition on $\varphi$, but on the fact that she has observed $\varphi$. Although we do require that $\varphi$ must be true if the agent observes it (BCS4), the agent may in general gain extra information by observing $\varphi$.

To understand this issue, consider the following example. Suppose that $\mathcal{R}$ is such that the agent observes $p_{1}$ at time $(r, m)$ only if $p_{2}$ and $q$ are also true at $(r, m)$, and she observes $p_{1} \wedge p_{2}$ at $(r, m)$ only if $q$ is false. It is easy to construct a BCS satisfying REV1-REV3 that also satisfies these requirements. In this system, after observing $p_{1}$, the agent believes $p_{2}$ and $q$. According to AGM's postulate R7 (and also KM's postulate U5) the agent must believe $q$ after observing $p_{1} \wedge p_{2}$. To see this, note that our assumptions about $\mathcal{R}$ can be phrased in the AGM language as $p_{2} \wedge q \in K \circ p_{1}$ and $\neg q \in K \circ\left(p_{1} \wedge p_{2}\right)$. Postulate R7 states that $K \circ\left(p_{1} \wedge p_{2}\right) \subseteq C l\left(K \circ p_{1} \cup\left\{p_{2}\right\}\right)$. Since $p_{2} \in K \circ p_{1}$, we have that $C l\left(K \circ p_{1} \cup\left\{p_{2}\right\}\right)=K \circ p_{1}$. Thus, R7 implies in this case that $q \in K \circ\left(p_{1} \wedge p_{2}\right)$. However, in $\mathcal{R}$, the agent believes (indeed knows) $\neg q$ after observing $p_{1} \wedge p_{2} .{ }^{8}$ Thus, revision and update

8. We stress this does not mean that $p_{1} \wedge p_{2}$ implies $\neg q$ in $\mathcal{R}$. There may well be points in $\mathcal{R}$ at which $p_{1} \wedge p_{2} \wedge q$ is true. However, at such points, the agent would not observe $p_{1} \wedge p_{2}$, since the agent observes $p_{1} \wedge p_{2}$ only if $q$ is false. 
both are implicitly assuming that the observation of $\varphi$ does not provide such additional knowledge. The following assumption ensures that this is the case for revision (a more general version will be required for update; see Section 6 ).

REV4 $\mathrm{Pl}_{a}\left(\mathcal{R}\left[\varphi ; o_{1}, \ldots, o_{m}\right]\right) \geq \mathrm{Pl}_{a}\left(\mathcal{R}\left[\psi ; o_{1}, \ldots, o_{m}\right]\right)$ if and only if $\mathrm{Pl}_{a}(\mathcal{R}[\varphi \wedge$ $\left.\left.o_{1} \wedge \ldots \wedge o_{m}\right]\right) \geq \mathrm{Pl}_{a}\left(\mathcal{R}\left[\psi \wedge o_{1} \wedge \ldots \wedge o_{m}\right]\right)$.

This assumption captures the intuition that observing $o_{1}, \ldots, o_{k}$ provides no more information than just the fact that $o_{1} \wedge \ldots \wedge o_{m}$ is true. That is, the agent compares the plausibility of $\varphi$ and $\psi$ in the same way after conditioning by the observations $o_{1}, \ldots, o_{m}$ as after conditioning by the fact that $o_{1} \wedge \ldots \wedge o_{m}$ is true. It easily follows from REV4 and PRIOR that the agent believes $\psi$ after observing $o_{1} \wedge \ldots \wedge o_{m}$ exactly if $o_{1} \wedge \ldots \wedge o_{m} \wedge \psi$ was initially considered more plausible than $o_{1} \wedge \ldots \wedge o_{m} \wedge \neg \psi$. Thus, the agent believes $\psi$ after observing $o_{1} \wedge \ldots \wedge o_{m}$ exactly if initially, she believed $\psi$ conditional on $o_{1} \wedge \ldots \wedge o_{m}$ : the observations provide no extra information beyond the fact that each of the $o_{i}$ 's are true.

REV4 is quite a strong assumption. Not only does it say that observations do not give the agent any additional information (beyond the fact that they are true), it also says that all consistent observations can be made (since if $\varphi \wedge o$ is consistent, we must have $\mathrm{Pl}_{a}(\mathcal{R}[\varphi ; o])=\mathrm{Pl}_{a}(\mathcal{R}[\varphi \wedge o])>\perp$, by REV3 and REV4). We might instead consider using a weaker version of REV4 that says that, provided an observation can be made, it gives no additional information. Formally, this would be captured as

REV4' If $\mathrm{Pl}_{a}\left(\mathcal{R}\left[\varphi ; o_{1}, \ldots, o_{m}\right]\right)>0$, then $\mathrm{Pl}_{a}\left(\mathcal{R}\left[\varphi ; o_{1}, \ldots, o_{m}\right]\right) \geq \mathrm{Pl}_{a}\left(\mathcal{R}\left[\psi ; o_{1}, \ldots, o_{m}\right]\right)$ if and only if $\mathrm{Pl}_{a}\left(\mathcal{R}\left[\varphi \wedge o_{1} \wedge \ldots \wedge o_{m}\right]\right) \geq \mathrm{Pl}_{a}\left(\mathcal{R}\left[\psi \wedge o_{1} \wedge \ldots \wedge \boldsymbol{o}_{m}\right]\right)$.

The following examples suggests that REV4' may be more reasonable in practice than REV4. We used REV4 only because it comes closer to the spirit of the requirement of revision that all observations are possible.

Example 5.1: Consider the system $\mathcal{I}_{\text {diag, } 1}$ described in Example 2.1. As discussed in Example 4.1, this system can be viewed as a BCS. Is it a revision system? It is easy to see that $\mathcal{I}_{\text {diag }, 1}$ satisfies REV2 and REV3. It clearly does not satisfy REV1, since propositions that describe input/output lines can change their values from one point to the next. However, as we are about to show, a slight variant of $\mathcal{I}_{\text {diag }, 1}$ does satisfy REV1. A more fundamental problem is that $\mathcal{I}_{\text {diag, } 1}$ does not satisfy REV4. This is inherent in our assumption that the agent never directly observes faults, so that, for example, we have $\mathrm{P} l_{\text {diag }, 1}\left(\mathcal{R}\left[\cdot ; f_{1}\right]\right)=\perp$, while $\mathrm{P} 1_{\text {diag, } 1}\left(\mathcal{R}\left[f_{1}\right]\right)>\perp$. It does, however, satisfy REV4'.

To see how to modify $\mathcal{I}_{\text {diag, } 1}$ so as to satisfy REV1, recall that in the diagnosis task, the agent is mainly interested in her beliefs about faults. Since faults are static in $\mathcal{I}_{\text {diag, } 1}$, we can satisfy REV1 if we ignore all propositions except $f_{1}, \ldots, f_{n}$. Let $\Phi_{\text {diag }}^{\prime}=\left\{f_{1}, \ldots, f_{n}\right\}$ and let $\mathcal{L}_{\text {diag }}^{\prime}$ be the propositional language over $\Phi_{\text {diag }}^{\prime}$. For every observation $o$ made by the agent regarding the value of the lines, there corresponds a formula in $\mathcal{L}_{\text {diag }}^{\prime}$ that characterizes all the fault sets that are consistent with $o$. Thus, for every run $r$ in $\mathcal{I}_{\text {diag, } 1}$, we can construct a run $r^{\prime}$ where the agent's local state is a sequence of formulas in $\mathcal{L}_{\text {diag }}^{\prime}$. Let $\mathcal{I}_{\text {diag }}^{\prime}$ be the system consisting of all such runs $r^{\prime}$. We can clearly put a plausibility assignment on these

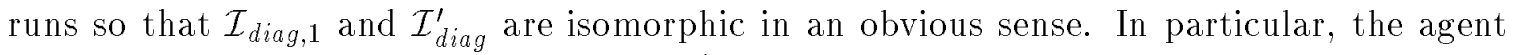
has the same beliefs about formulas in $\mathcal{L}_{\text {diag }}^{\prime}$ at corresponding points in the two systems. 
More precisely, if $\varphi \in \mathcal{L}_{\text {diag }}^{\prime}$, then $\left(\mathcal{I}_{\text {diag }}^{\prime}, r, m\right) \models \varphi$ if and only if $\left(\mathcal{I}_{\text {diag, }, 1}, r, m\right) \models \varphi$ for all points $(r, m)$ in $\mathcal{I}_{\text {diag, } 1}$. It is easy to verify that $\mathcal{I}_{\text {diag }}^{\prime}$ satisfies REV1-REV3 and REV4', although it still does not satisfy REV4.

We are not advocating here here using $\mathcal{I}_{\text {diag }}^{\prime}$ instead of $\mathcal{I}_{\text {diag }}-\mathcal{I}_{\text {diag }}$ seems to us a perfectly reasonable way of modeling the situation. Rather, the point is that if we want a BCS to satisfy properties that validate the AGM postulates, we must make some strong, and not always natural, assumptions.

We want to show that a revision operator corresponds to a system in $\mathcal{C}^{R}$ and vice versa. To do so, we need to examine the beliefs of the agent at each point $(r, m)$. First we note that if $(r, m) \sim_{a}\left(r^{\prime}, m^{\prime}\right)$ then $(\mathcal{I}, r, m) \models B \varphi$ if and only if $\left(\mathcal{I}, r^{\prime}, m^{\prime}\right) \models B \varphi$; this is a consequence of the requirement that, as we have defined interpreted systems, the agent's plausibility assessment is a function of her local state. Thus, we think of the agent's beliefs as a function of her local state. We use the notation $\left(\mathcal{I}, s_{a}\right) \models B \varphi$ as shorthand for $(\mathcal{I}, r, m) \models B \varphi$ for some $(r, m)$ such that $r_{a}(m)=s_{a}$. Let $s_{a}$ be some local state of the agent. We define the agent's belief state at $s_{a}$ as

$$
\operatorname{Bel}\left(\mathcal{I}, s_{a}\right)=\left\{\varphi \in \mathcal{L}_{e}:\left(\mathcal{I}, s_{a}\right) \models B \varphi\right\} .
$$

Since the agent's state is a sequence of observations, the agent's state after observing $\varphi$ is simply $s_{a} \cdot \varphi$, where $\cdot$ is the append operation. Thus, $\operatorname{Bel}\left(\mathcal{I}, s_{a} \cdot \varphi\right)$ is the belief state after observing $\varphi$. We adopt the convention that if the agent can never attain the local state $s_{a}$ in $\mathcal{I}$, then $\operatorname{Bel}\left(\mathcal{I}, s_{a}\right)=\mathcal{L}_{e}$. With these definitions, we can compare the agent's belief state before and after observing $\varphi$, that is $\operatorname{Bel}\left(\mathcal{I}, s_{a}\right)$ and $\operatorname{Bel}\left(\mathcal{I}, s_{a} \cdot \varphi\right)$.

We start by showing that every AGM revision operator can be represented in $\mathcal{C}^{R}$.

Theorem 5.2: Let $\circ$ be an $A G M$ revision operator and let $K \subseteq \mathcal{L}_{e}$ be a consistent belief state. Then there is a system $\mathcal{I}_{\circ, K} \in \mathcal{C}^{R}$ such that $\operatorname{Bel}\left(\mathcal{I}_{\circ, K},\langle\rangle\right)=K$ and

$$
\operatorname{Bel}\left(\mathcal{I}_{\circ, K},\langle\rangle\right) \circ \varphi=\operatorname{Bel}\left(\mathcal{I}_{\circ, K},\langle\varphi\rangle\right)
$$

for all $\varphi \in \mathcal{L}_{e}$.

Proof: See Appendix A.1.

Thus, Theorem 5.2 says that we can represent a revision operator $\circ$ in the sense that we have a family of systems $\mathcal{I}_{0, K} \in \mathcal{C}^{R}$, one for each consistent belief state $K$, such that $K$ is the agent's initial belief state in $\mathcal{I}_{o, K}$, and for each formula $\varphi$ in $\mathcal{L}_{e}$, the agent's belief state after learning $\varphi$ is $K \circ \varphi$. Notice that we restrict attention to consistent belief states $K$. The AGM postulates allow the agent to "escape" from an inconsistent state, so that $K \circ \varphi$ may be consistent even if $K$ is inconsistent. We might thus hope to extend the theorem so that it also applies to the inconsistent belief state, but this is impossible in our framework. If false $\in \operatorname{Bel}\left(\mathcal{I}_{o, K}, s_{a}\right)$ for some state $s_{a}$, and $r_{a}(m)=s_{a}$, then $\mathrm{Pl}_{(r, m)}\left(W_{(r, m)}\right)=\perp$. Since we update by conditioning, we must have $\mathrm{Pl}_{(r, m+1)}\left(W_{(r, m+1)}\right)=\perp$, so the agent's belief state will remain inconsistent no matter what she learns. Although we could modify our framework to allow the agent to escape from inconsistent states, we actually consider this to be a defect in the AGM postulates, not in our framework. To see why, suppose that the 
agent's belief set is inconsistent at $s_{a}$, and $r_{a}(m)=s_{a}$. Thus, the agent considers all states in $W_{(r, m)}$ to be completely implausible $\left(\right.$ since $\mathrm{Pl}(r, m)\left(W_{(r, m)}\right)=\perp$ ). On the other hand, to escape inconsistency, she must have a plausibility ordering over the worlds in $W_{(r, m)}$. These two requirements seem somewhat inconsistent. ${ }^{9}$

Not surprisingly, this inconsistency creates problems for other semantic representations in the literature. For example, Boutilier's representation theorem (1992) states that for every revision operator $\circ$ and belief state $K$, there is a ranking $R$ such that $\psi \in K \circ \varphi$ if and only if $\psi$ is believed in the minimal $\varphi$-worlds according to $R$. If we examine this theorem, we note that he does not state that the minimal (i.e., most preferred) worlds in $R$ correspond to the belief state $K$ (in the sense that the minimal worlds are precisely those where the formulas in $K$ hold); this would be the analogue of our requiring that $\operatorname{Bel}\left(\mathcal{I}_{\circ, K},\langle\rangle\right)=K$. In fact, if $K$ is $\vdash_{\mathcal{L}_{e}}$-consistent, the minimal worlds do correspond to $K$. However, if $K$ is inconsistent, they cannot, since any nonempty ranking induces a consistent set of beliefs. We could state a weaker version of Theorem 5.2 that would correspond exactly to Boutilier's theorem. We presented the stronger result (that does not apply to inconsistent belief states) to bring out what we believe to be a problem with the AGM postulates. See (Friedman \& Halpern, 1998a) for further discussion of this issue.

Theorem 5.2 shows that, in a precise sense, we can map AGM revision operations to $\mathcal{C}^{R}$. What about the other direction? The next theorem shows that the first belief change step in systems in $\mathcal{C}^{R}$ satisfies the AGM postulates.

Theorem 5.3: Let $\mathcal{I}$ be a system in $\mathcal{C}^{R}$. Then there is an $A G M$ revision operator ${ }^{\circ} \mathcal{I}$ such that

$$
\operatorname{Bel}\left(\mathcal{I},\langle\rangle \circ_{\mathcal{I}} \varphi=\operatorname{Bel}(\mathcal{I},\langle\varphi\rangle)\right.
$$

for all $\varphi \in \mathcal{L}_{e}$.

Proof: See Appendix A.1.

We remark that if we used REV4' instead of REV4, then we would be able to prove this result only for those formulas $\varphi$ that are observable (i.e., for which $\operatorname{Pl}(\mathcal{R}[\varphi])>\perp$ ).

Both Theorems 5.2 and 5.3 apply to one-step revision, starting from the initial (empty) state. What happens once we allow iterated revision? In our framework, observations are taken to be known, so if the agent makes an inconsistent sequence of observations, then her belief state will be inconsistent, and (as we observed above) will remain inconsistent from then on, no matter what she observes. This creates a problem if we try to get analogues to Theorems 5.2 and 5.3 for iterated revision. As the following theorem demonstrates, we can already see the problem if we consider one-step revisions from a state other than the initial state.

Theorem 5.4: Let $\mathcal{I}$ be a system in $\mathcal{C}^{R}$ and let $s_{a}=\left\langle\varphi_{1}, \ldots, \varphi_{k}\right\rangle$ be a local state in $\mathcal{I}$. Then there is an $A G M$ revision operator ${ }^{\circ} \mathcal{I}_{, s_{a}}$ such that

$$
\operatorname{Bel}\left(\mathcal{I}, s_{a}\right){ }_{\mathcal{I}, s_{a}} \varphi=\operatorname{Bel}\left(\mathcal{I}, s_{a} \cdot \varphi\right)
$$

9. One strength of the AGM framework is that it can deal with an inconsistent sequence of observations, that is, it can cope with an observation sequence of the form $\langle p, \neg p, p, \neg p, \ldots\rangle$. We stress that being able to cope with such an inconsistent sequence of observations does not require allowing the agent to escape from inconsistent belief sets. These are two orthogonal issues. 
for all formulas $\varphi \in \mathcal{L}_{e}$ such that $\varphi_{1} \wedge \ldots \wedge \varphi_{k} \wedge \varphi$ is consistent.

Proof: See Appendix A.1.

We cannot do better than this. If $\varphi_{1} \wedge \ldots \wedge \varphi_{k} \wedge \varphi$ is inconsistent then, because of our requirements that all observations must be true of the current state of the environment (BCS4) and that propositions are static (REV1), there cannot be any global state in $\mathcal{I}$ where the agent's local state in $s_{a} \cdot \varphi$. Thus, $\operatorname{Bel}\left(\mathcal{I}, s_{a} \cdot \varphi\right)$ is inconsistent, contradicting R5.

There is another problem with trying to get an analogue of Theorem 5.3 for iterated revision, a problem that seems inherent in the AGM framework. Our framework makes a clear distinction between the agent's epistemic state at a point $(r, m)$ in $\mathcal{I}$, which we can identify with her local state $s_{a}=r_{a}(m)$, and the agent's belief set at $(r, m), \operatorname{Bel}\left(\mathcal{I}, s_{a}\right)$, which is the set of formulas she believes. In a system in $\mathcal{C}^{R}$, the agent's belief set does not in general determine how the agent's beliefs will be revised; her epistemic state does. On the other hand, the AGM postulates assume that revision is a function of the agent's belief set and observations. Now suppose we have a system $\mathcal{I}$ and two points $(r, m)$ and $\left(r, m^{\prime}\right)$ on some run $r \in \mathcal{I}$ such that (1) the agent's belief set is the same at $(r, m)$ and $\left(r, m^{\prime}\right)$, that is $\operatorname{Bel}\left(\mathcal{I}, r_{a}(m)\right)=\operatorname{Bel}\left(\mathcal{I}, r_{a}\left(m^{\prime}\right)\right)$, (2) the agent observes $\varphi$ at both $(r, m)$ and $\left(r, m^{\prime}\right),(3) \operatorname{Bel}\left(\mathcal{I}, r_{a}(m+1)\right) \neq \operatorname{Bel}\left(\mathcal{I}, r_{a}\left(m^{\prime}+1\right)\right.$. It is not hard to construct such a system $\mathcal{I}$. However, there cannot be an analogue of Theorem 5.3 for $\mathcal{I}$, even if we restrict to consistent sequences of observations. For suppose there were a revision operator $\circ$ such $\operatorname{Bel}(\mathcal{I},\langle\rangle)) \circ \varphi_{1} \circ \cdots \circ \varphi_{k}=\operatorname{Bel}\left(\mathcal{I},\left\langle\varphi_{1}, \ldots, \varphi_{k}\right\rangle\right)$ for all $\varphi_{1}, \ldots, \varphi_{k}$ such that $\varphi_{1} \wedge \ldots \wedge \varphi_{k}$ is consistent. Then we would have $\operatorname{Bel}\left(\mathcal{I}, r_{a}(m+1)\right)=\operatorname{Bel}\left(\mathcal{I}, r_{a}(m)\right) \circ \varphi=\operatorname{Bel}\left(\mathcal{I}, r_{a}\left(m^{\prime}\right)\right) \circ \varphi=$ $\operatorname{Bel}\left(\mathcal{I}, r_{a}\left(m^{\prime}+1\right)\right)$, contradicting our assumption.

The culprit here is the assumption that revision depends only on the agent's belief set. To see why this is an unreasonable assumption, consider a situation where at time 0 the agent believes both $p$ and $q$, but her belief in $q$ is stronger than her belief in $p$ (i.e., the plausibility of $q$ is greater than that of $p$ ). We can well imagine that after observing $\neg p \vee \neg q$ at time 1 , she would believe $\neg p$ and $q$. However, if she first observed $p$ at time 1 and then $\neg p \vee \neg q$ at time 2 , she would believe $p$ and $\neg q$, because, as a result of observing $p$, she would assign $p$ greater plausibility than $q$. Note, however, that the AGM postulates dictate that after an observation that is already believed, the agent does not change her beliefs. Thus, the AGM setup would force the agent to have the same beliefs after learning $\neg p \vee \neg q$ in both situations.

There has been a great deal of work on the problem of iterated belief revision (Boutilier, 1996a; Darwiche \& Pearl, 1997; Freund \& Lehmann, 1994; Lehmann, 1995; Levi, 1988; Nayak, 1994; Williams, 1994)). Much of the recent work moves away from the assumption that belief revision depends solely on the agent's belief set. For example the approaches of Boutilier (1996a) and Darwiche and Pearl (1997) define revision operators that map (rankings $\times$ formulas) to rankings. Because our framework makes such a clear distinction between epistemic states and belief states, it gives us a natural way of maintaining the spirit of the AGM postulates while assuming that revision is a function of epistemic states. Rather than taking 0 to be a function from (belief states $\times$ formulas) to belief states, we take it 0 to be a function from (epistemic states $x$ formulas) to epistemic states.

This leaves open the question of how to represent epistemic states. Boutilier and Darwiche and Pearl use rankings to represent epistemic states. In our framework, we represent 
epistemic states by local states in interpreted systems. That is, a pair $\left(\mathcal{I}, s_{a}\right)$ denotes the agent's state in an interpreted system, and the pair determines the agent's relevant epistemic attitudes, such as her beliefs, how her beliefs changed given particular observations, her plausibility assessment over runs, and so on. When the system is understood, we simply use $s_{a}$ as a shorthand representation of an epistemic state.

We can easily modify the AGM postulates to deal with such revision operators on epistemic states. We start by assuming that there is a set of epistemic states and a function $\operatorname{Bel}(\cdot)$ that maps epistemic states to belief states. We then have analogues to each of the AGM postulates, obtained by replacing each belief set by the beliefs in the corresponding epistemic state. For example, we have

( $\left.\mathbf{R} 1^{\prime}\right) E \circ \varphi$ is an epistemic state

$\left(\mathbf{R 2}^{\prime}\right) \varphi \in \operatorname{Bel}(E \circ \varphi)$

$\left(\mathbf{R 3}^{\prime}\right) \operatorname{Bel}(E \circ \varphi) \subseteq C l(\operatorname{Bel}(E) \cup\{\varphi\})$

and so on, with the obvious transformation. ${ }^{10}$

We can get strong representation theorems if we work at the level of epistemic states. Given a language $\mathcal{L}_{e}$ (with an associated consequence relation $\vdash_{\mathcal{L}_{e}}$ ), let $\mathcal{E}_{\mathcal{L}_{e}}$ consist of all finite sequences of formulas in $\mathcal{L}_{e}$. Note that we allow $\mathcal{E}_{\mathcal{L}_{e}}$ to include sequences of formulas whose conjunction is inconsistent. We define revision in $\mathcal{E}_{\mathcal{L}_{e}}$ in the obvious way: if $E \in \mathcal{E}_{\mathcal{L}_{e}}$, then $E \circ \varphi=E \cdot \varphi$.

Theorem 5.5: Let $\mathcal{I}$ be a system in $\mathcal{C}^{R}$ whose local states are $\mathcal{E}_{\mathcal{L}_{e}}$. There is a function Bel $l_{\mathcal{I}}$ that maps epistemic states to belief states such that

- if $s_{a}$ is a local state of the agent in $\mathcal{I}$, then $\operatorname{Bel}\left(\mathcal{I}, s_{a}\right)=\operatorname{Bel}_{\mathcal{I}}\left(s_{a}\right)$, and

- $\left(\mathrm{o}, B e l_{\mathcal{I}}\right)$ satisfies $R 1^{\prime}-R 8^{\prime}$.

Proof: Roughly speaking, we define $\operatorname{Bel}_{\mathcal{I}}\left(s_{a}\right)=\operatorname{Bel}\left(\mathcal{I}, s_{a}\right)$ when $s_{a}$ is a local state in $\mathcal{I}$. If $s_{a}$ is not in $\mathcal{I}$, then we set $\operatorname{Bel}_{\mathcal{I}}\left(s_{a}\right)=\operatorname{Bel}\left(\mathcal{I}, s^{\prime}\right)$, where $s^{\prime}$ is the longest consistent suffix of $s_{a}$. See Appendix A.1 for details.

Notice that, by definition, we have $\operatorname{Bel}_{\mathcal{I}}\left(\mathcal{I},\langle\rangle \circ_{\mathcal{I}} \varphi_{1} \circ_{\mathcal{I}} \ldots \circ_{\mathcal{I}} \varphi_{k}\right)=\operatorname{Be} l_{\mathcal{I}}\left(\mathcal{I},\left\langle\varphi_{1}, \ldots, \varphi_{k}\right\rangle\right)$, so, at the level of epistemic states, we get an analogue to Theorem 5.3. We remark that to ensure that $\mathrm{R} 5^{\prime}$ holds for $\left(\circ, \operatorname{Bel}_{\mathcal{I}}\right)$, we need to define $\operatorname{Be}_{\mathcal{I}}(E)$ appropriately for sequences $E \in \mathcal{E}_{\mathcal{I}}$ whose conjunction is inconsistent.

Theorem 5.5 shows that any system in $\mathcal{C}^{R}$ corresponds to a revision operator over epistemic states that satisfies the generalized AGM postulates. We would hope that the converse also holds. Unfortunately, this is not quite the case. There are revision operators on epistemic states that satisfy the generalized AGM postulates but do not correspond to a system in $\mathcal{C}^{R}$. This is because systems in $\mathcal{C}^{R}$ satisfy an additional postulate:

$\left(\mathbf{R 9}^{\prime}\right)$ If $\nvdash_{\mathcal{L}_{e}} \neg(\varphi \wedge \psi)$ then $\operatorname{Bel}(E \circ \varphi \circ \psi)=\operatorname{Bel}(E \circ \varphi \wedge \psi)$.

10. The only problematic postulate is R6. The question is whether R6' should be "If $\vdash_{\mathcal{L}_{e}} \varphi \Leftrightarrow \psi$ then $\operatorname{Bel}(E \circ \varphi)=\operatorname{Bel}(E \circ \psi)$ " or "If $\vdash_{\mathcal{L}_{e}} \varphi \Leftrightarrow \psi$ then $E \circ \varphi=E \circ \psi "$. Dealing with either version is straightforward. For definiteness, we adopt the first alternative here. 
We show that $R 9^{\prime}$ is sound in $\mathcal{C}^{R}$ by proving the following strengthening of Theorem 5.5.

Proposition 5.6: Let $\mathcal{I}$ be a system in $\mathcal{C}^{R}$ whose local states are $\mathcal{E}_{\mathcal{L}_{e}}$. There is a function $B$ Bel $l_{\mathcal{I}}$ that maps epistemic states to belief states such that

- if $s_{a}$ is a local state of the agent in $\mathcal{I}$, then $\operatorname{Bel}\left(\mathcal{I}, s_{a}\right)=\operatorname{Be} l_{\mathcal{I}}\left(s_{a}\right)$, and

- (o,Beli ) satisfies $R t^{\prime}-R g^{\prime}$.

Proof: We show that the function $\mathrm{Bel}_{\mathcal{I}}$ defined in the proof of Theorem 5.5 satisfies $\mathrm{R} 9$ '. See Appendix A.1 for details.

We can prove the converse to Proposition 5.6: a revision system on epistemic states that satisfies the generalized AGM postulates and $R g^{\prime}$ does correspond to a system in $\mathcal{C}^{R}$.

Theorem 5.7: Given a function Bel $\mathcal{L}_{\mathcal{L}_{e}}$ mapping epistemic states in $\mathcal{E}_{\mathcal{L}_{e}}$ to belief sets over $\mathcal{L}_{e}$ such that Bel $\mathcal{L}_{\mathcal{L}_{e}}(\langle\rangle)$ is consistent and $\left(\right.$ Bel $\left._{\mathcal{L}_{e}}, \circ\right)$ satisfies $R 1^{\prime}-R g^{\prime}$, there is a system $\mathcal{I} \in \mathcal{C}^{R}$ whose local states are in $\mathcal{E}_{\mathcal{L}_{e}}$ such that Bel $\mathcal{L}_{\mathcal{L}_{e}}\left(s_{a}\right)=\operatorname{Bel}\left(s_{a}\right)$ for each local state $s_{a}$ in $\mathcal{I}$.

Proof: According to Theorem 5.2, there is a system $\mathcal{I}$ such that $\operatorname{Bel}(\mathcal{I},\langle\rangle)=\operatorname{Bel}_{\mathcal{L}_{e}}(\langle\rangle)$ and $\operatorname{Bel}(\mathcal{I},\langle\varphi\rangle)=\operatorname{Bel}_{\mathcal{L}_{e}}(\langle\varphi\rangle)$ for all $\varphi \in \mathcal{L}_{e}$. We show that $\operatorname{Bel}\left(\mathcal{I}, s_{a}\right)=\operatorname{Bel}_{\mathcal{L}_{e}}\left(s_{a}\right)$ for local states $s_{a}$ in $\mathcal{I}$. See Appendix A.1. $\square$

Notice that, by definition, for the system $\mathcal{I}$ of Theorem 5.7, we have $\operatorname{Bel}\left(\langle\rangle \circ \varphi_{1} \circ \ldots \circ \varphi_{k}\right)=$ $\operatorname{Bel}\left(\left\langle\varphi_{1}, \ldots, \varphi_{k}\right\rangle\right)$ as long as $\varphi_{1} \wedge \ldots \wedge \varphi_{k}$ is consistent.

\section{Capturing Update}

Update tries to capture the intuition that there is a preference for runs where all the observations made are true, and where changes from one point to the next along the run are minimized.

We start by reviewing Katsuno and Mendelzon's semantic representation of update. To characterize an agent beliefs, Katsuno and Mendelzon consider the set of "worlds" the agent considers possible. In their representation, they associate a world with a truth assignment to the primitive propositions. (In our terminology, we can think of a world as an environment state.) To capture the notion of "minimal change from world to world", Katsuno and Mendelzon use a distance function $d$ on worlds. Given two worlds $w$ and $w^{\prime}, d\left(w, w^{\prime}\right)$ measures the distance between them. Intuitively, the larger the distance, the larger the change required to get from world $w$ to $w^{\prime}$. (Note that that distances are not necessarily symmetric, that is, it might require a smaller change to get from $w$ to $w^{\prime}$, than from $w^{\prime}$ to $w$.) Distances might be incomparable, so we require that $d$ map pairs of worlds into a partially ordered domain with a unique minimal element 0 and that $d\left(w, w^{\prime}\right)=0$ if and only if $w=w^{\prime}$.

Katsuno and Mendelzon show that there is a close relationship between update operators and distance functions. To make this relationship precise, we need to introduce some definitions. An update structure is a tuple $U=(W, d, \pi)$, where $W$ is a finite set of worlds, $d$ is a distance function on $W$, and $\pi$ is a mapping from worlds to truth assignments for $\mathcal{L}_{e}$ such that

- $\pi(w)$ is $\vdash_{\mathcal{L}_{e}}$ consistent, 
- if $\forall \mathcal{L}_{e} \neg \varphi$, then there is some $w \in W$ with $\pi(w)(\varphi)=$ true, and

- if $w \neq w^{\prime}$ then $\pi(w) \neq \pi\left(w^{\prime}\right)$ for all $w, w^{\prime} \in W$.

Given an update structure $U=(W, d, \pi)$, we define $\llbracket \varphi \rrbracket U=\{w: \pi(w)(\varphi)=$ true $\}$. Katsuno and Mendelzon use update structures as semantic representations of update operators. Given an update structure $U=(W, d, \pi)$ and sets $A, B \subseteq W$, Katsuno and Mendelzon define $\min _{U}(A, B)$ to be the set of worlds in $B$ that are closest to worlds in $A$, according to d. Formally, $\min _{U}(A, B)=\left\{w \in B: \exists w_{0} \in A \forall w^{\prime} \in B d\left(w_{0}, w^{\prime}\right) \nless d\left(w_{0}, w\right)\right\}$.

Theorem 6.1: (Katsuno \& Mendelzon, 1991b) A belief change operator $\diamond$ satisfies U1-U8 if and only if there is an update structure $U=(W, \pi, d)$ such that

$$
\llbracket \varphi \diamond \psi \rrbracket_{U}=\min _{U}\left(\llbracket \varphi \rrbracket_{U}, \llbracket \psi \rrbracket_{U}\right)
$$

Thus the worlds the agent believes possible after updating with $\psi$ are these worlds that are closest to some world considered possible before learning $\psi$.

Katsuno and Mendelzon's account of update is "static" in the sense that it describes a single belief change. Nevertheless, there is a clear intuition that each world $w^{\prime} \in \llbracket \varphi \diamond \psi \rrbracket_{U}$ is the result of considering a minimal change from some world $w \in \llbracket \varphi \rrbracket U$. However, in Katsuno and Mendelzon's representation, we do not keep track of the worlds that "lead to" the worlds in the current belief set.

We now try to capture behavior similar to Katsuno and Mendelzon's semantics in our framework. We define systems where each run describes the sequence of changes, so that the most plausible runs, given a set of observations, correspond the worlds that define the belief set in Katsuno and Mendelzon's semantics. More precisely, given a sequence of observations $\psi_{1}, \ldots, \psi_{n}$, each world in $\llbracket \varphi \diamond \psi_{1} \diamond \ldots \diamond \psi_{n} \rrbracket_{U}$ can be "traced" back through a series of minimal changes to a world in $\llbracket \varphi \rrbracket_{U}$. In our model, each such trace corresponds to one of the most plausible runs, where the environment state at time $m$ is the $m$ th world in the trace. We can capture this intuition by using a family of priors with a particular form.

We start with some preliminary definitions. Let $\mathcal{I}$ be a BCS, and let $s_{0}, \ldots, s_{n}$ be a set of environment states in $\mathcal{I}$. We define $\left[s_{0}, \ldots, s_{n}\right]$ as the set of runs where $r_{e}(i)=s_{i}$ for all $0 \leq i \leq n$. Thus, $\left[s_{0}, \ldots, s_{n}\right]$ describes a set of runs that share a common prefix of environment states. A prior plausibility space $\mathcal{P}_{a}=\left(\mathcal{R}, \mathrm{P} l_{a}\right)$ is consistent with a distance measure $d$ if the following holds:

$$
\begin{aligned}
& \mathrm{P} l_{a}\left(\left[s_{0}, \ldots, s_{n}\right]\right)<\mathrm{P} 1_{a}\left(\left[s_{0}^{\prime}, \ldots, s_{n}^{\prime}\right]\right) \text { if and only if there is some } j<n \text { such that } \\
& s_{k}=s_{k}^{\prime} \text { for all } 0 \leq k \leq j, s_{j+1} \neq s_{j+1}^{\prime} \text {, and } d\left(s_{j}, s_{j+1}\right)<d\left(s_{j}, s_{j+1}^{\prime}\right) .
\end{aligned}
$$

Intuitively, we compare events of the form $\left[s_{0}, \ldots, s_{n}\right]$ using a lexicographic ordering based on $d$. Notice that this ordering focuses on the first point of difference. Runs with a smaller change at this point are preferred, even if later there are abnormal changes. This point is emphasized in the borrowed car example below.

$\mathrm{Pl}_{a}$ is prefix-defined if the plausibility of an event is uniquely defined by the plausibility of run-prefixes that are contained in it, so that

$\mathrm{Pl}_{a}\left(\mathcal{R}\left[\varphi_{0}, \ldots, \varphi_{m}\right]\right) \geq \mathrm{Pl}_{a}\left(\mathcal{R}\left[\psi_{0}, \ldots, \psi_{m}\right]\right)$ if and only if for all $\left[s_{0}, \ldots, s_{m}\right] \subseteq$ $\mathcal{R}\left[\psi_{0}, \ldots, \psi_{m}\right] \Leftrightarrow \mathcal{R}\left[\varphi_{0}, \ldots, \varphi_{m}\right]$ there is some $\left[s_{0}^{\prime}, \ldots, s_{m}^{\prime}\right] \subseteq \mathcal{R}\left[\varphi_{0}, \ldots, \varphi_{m}\right]$ such that $\mathrm{Pl}_{a}\left(\left[s_{0}^{\prime}, \ldots, s_{m}^{\prime}\right]\right)>\mathrm{Pl}_{a}\left(\left[s_{0}, \ldots, s_{m}\right]\right)$. 
Roughly speaking, this requirement states that we compare events by properties of dominance. This property is similar to one satisfied by the plausibility measures that we get from preference ordering using the construction of Proposition 2.2.

We define the set $\mathcal{C}^{U}$ to consist of $\operatorname{BCSs} \mathcal{I}=(\mathcal{R}, \pi, \mathcal{P})$ that satisfy the following four requirements UPD1-UPD4. UPD1 says that there are only finitely many possible truth assignments, and that there is a one-to-one map between environment states and truth assignments.

UPD1 The set $\Phi_{e}$ of propositions (of BCS1) is finite and $\pi$ is such that for all environment states $s, s^{\prime}$, if $s \neq s^{\prime}$, then there is a formula $\varphi \in \mathcal{L}_{e}$ such that $s \models \varphi$ and $s^{\prime} \models \neg \varphi$.

UPD2-UPD4 are analogues to REV2-REV4. Like REV2, UPD2 puts constraints on the form of the prior, but now we consider lexicographic priors of the form described above.

UPD2 The prior of BCS5 is prefix defined and consistent with some distance measure.

Recall that REV3 requires only that all truth assignments initially have nontrivial plausibility. In the case of revision, the truth assignment does not change over time, since we are dealing with static propositions. In the case of update, the truth assignment may change over time, so UPD3 requires that all consistent sequences of truth assignments have nontrivial plausibility.

UPD3 If $\varphi_{i} \in \mathcal{L}_{e}, i=0, \ldots, k$, are consistent formulas, then $\operatorname{Pl}\left(\mathcal{R}\left[\varphi_{0}, \ldots, \varphi_{k}\right]\right)>$ $\perp$.

Finally, like REV4, UPD4 requires that the agent gain no information from her observations beyond the fact that they are true.

UPD4 $\mathrm{Pl}_{a}\left(\mathcal{R}\left[\varphi_{0}, \ldots, \varphi_{k+1} ; o_{1}, \ldots, o_{k}\right]\right) \geq \mathrm{Pl}_{a}\left(\mathcal{R}\left[\psi_{0}, \ldots, \psi_{m+1} ; o_{1}, \ldots, o_{m}\right]\right)$ if and only if $\mathrm{Pl}_{a}\left(\mathcal{R}\left[\varphi_{0}, \varphi_{1} \wedge o_{1}, \ldots, \varphi_{m} \wedge o_{m}, \varphi_{m+1}\right]\right) \geq \mathrm{Pl}_{a}\left(\mathcal{R}\left[\psi_{0}, \psi_{1} \wedge o_{1}, \ldots, \psi_{m} \wedge\right.\right.$ $\left.\boldsymbol{o}_{m}, \psi_{m+1}\right]$ )

We remark that in the presence of REV1, UPD4 is equivalent to REV4. We might consider generalized versions of UPD4, where the two sequences of formulas can have arbitrary relative lengths; this version suffices for our purposes. We can also define an analogue UPD4' in the spirit of REV4', which applies only if $\mathrm{Pl}\left(\mathcal{R}\left[\varphi_{0}, \ldots, \varphi_{m+1} ; o_{1}, \ldots, o_{m}\right]\right)>\perp$.

We now show that $\mathcal{C}^{U}$ corresponds to (KM) update. Recall that Katsuno and Mendelzon define an update operator as mapping a pair of formulas $(\mu, \varphi)$, where $\mu$ describes the agent's beliefs and $\varphi$ describes the observation, to a new formula $\mu \diamond \varphi$ that describes the agent's new beliefs. However, as we discussed in Section 3 , when $\Phi_{e}$ is finite, we can also treat $\diamond$ mapping a belief state and a formula to a new belief state. Also recall that $\operatorname{Bel}\left(\mathcal{I}, s_{a}\right)$ is the agent's belief set when her local state is $s_{a}$.

Theorem 6.2: A belief change operator $\diamond$ satisfies U1-U8 if and only if there is a system $\mathcal{I} \in \mathcal{C}^{U}$ such that

$$
\operatorname{Bel}\left(\mathcal{I}, s_{a}\right) \diamond \psi=\operatorname{Bel}\left(\mathcal{I}, s_{a} \cdot \psi\right)
$$

for all epistemic states $s_{a}$ and formulas $\psi \in \mathcal{L}_{e}$. 
Proof: Roughly speaking, we show that any system in $\mathcal{C}^{U}$ corresponds to a Katsuno and Mendelzon update structure. Suppose that $\mathcal{I}=\in \mathcal{C}^{U}$ is such that the set of environment states is $\mathcal{S}_{e}$ and the prior of BCS5 is consistent with distance function $d$. We define an update structure $U_{\mathcal{I}}$. We then show that belief change in $\mathcal{I}$ corresponds to belief change in $U_{\mathcal{I}}$ in the sense of Theorem 6.1. Since Theorem 6.1 states that any belief change operation defined by an update structure satisfies $\mathrm{U} 1-\mathrm{U} 8$, this will suffice to prove the "if" direction of the theorem. To prove the "only if" direction of the theorem, we show that that for any update structure $U$, there is a system $\mathcal{I} \in \mathcal{C}^{U}$ such that $U_{\mathcal{I}}=U$.

See Appendix A.2 for details.

This result immediately generalizes to sequences of updates.

Corollary 6.3: A belief change operator $\diamond$ satisfies U1-U8 if and only if there is a system $\mathcal{I}_{\diamond} \in \mathcal{C}^{U}$ such that for all $\psi_{1}, \ldots, \psi_{k} \in \mathcal{L}_{e}$, we have

$$
\operatorname{Bel}\left(\mathcal{I}_{\diamond}, s_{a}\right) \diamond \psi_{1} \diamond \ldots \diamond \psi_{k}=\operatorname{Bel}\left(\mathcal{I}_{\diamond}, s_{a} \cdot \psi_{1} \cdot \ldots \cdot \psi_{k}\right) .
$$

These results show that for update, unlike revision, the systems we consider are such that the belief state does determine the result of the update, i.e., if $\operatorname{Bel}\left(\mathcal{I}, s_{a}\right)=\operatorname{Bel}\left(\mathcal{I}, s_{a}^{\prime}\right)$, then for any $\varphi$ we get that $\operatorname{Bel}\left(\mathcal{I}, s_{a} \cdot \varphi\right)=\operatorname{Bel}\left(\mathcal{I}, s_{a}^{\prime} \cdot \varphi\right)$. Roughly speaking, the reason is that the distance measure that determines the prior does not change over time. While this allows us to get an elegant representation theorem, it also causes problems for the applicability of update, as we shall see below.

Note that, since the world is allowed to change, there is no problem if we update by a sequence $\psi_{1}, \ldots, \psi_{k}$ of consistent formulas such that $\psi_{1} \wedge \ldots \wedge \psi_{k}$ is inconsistent. There is no requirement that the formulas $\psi_{1}, \ldots, \psi_{k}$ be true simultaneously. All that matters is that $\psi_{i}$ is true at time $i$. Also note that an update by an inconsistent formula does not pose a problem for our framework. It follows from postulates U1 and U2 that once the agent learns an inconsistent formula (i.e., false), she believes false from then on.

How reasonable is the notion of update? As the discussion of UPD2 above suggests, it has a preference for deferring abnormal events. This makes it quite similar to Shoham's chronological ignorance (1988), and it suffers from some of the same problems. Consider the following story, that we call the borrowed-car example. ${ }^{11}$ At time 1, the agent parks her car in front of her house with a full fuel tank. At time 2, she is in her house. At time 3, she returns outside to find the car still parked where she left it. Since the agent does not observe the car while she is inside the house, there is no reason for her to revise her beliefs regarding the car's location. Since she finds it parked at time 3 , she still has no reason to change her beliefs. Now, what should the agent believe when, at time 4, she notices that the fuel tank is no longer full? The agent may want to consider a number of possible explanations for her time-4 observation, depending on what she considers to be the most likely sequence(s) of events between time 1 and time 4 . For example, if she has had previous gas leaks, then she may consider leakage to be the most plausible explanation. On the other hand, if her spouse also has the car keys, she may consider it possible that he used the car in her absence. Update, however, prefers to defer abnormalities, so it will conclude that the

11. This example is based on Kautz's stolen car story (1986), and is due to Boutilier, who independently observed this problem [private communication, 1993]. 
fuel must have disappeared, for inexplicable reasons, between times 3 and 4 . To see this, note that runs where the car has been taken on a ride have an abnormality at time 2, while runs where the car did not move at time 2 but the fuel suddenly disappeared, have their first abnormality at time 4 , and thus are preferred!

Suppose we formalize the example using propositions such as car-parked-outside, fueltank-full, etc. Let the agent's belief set at time $i$ be $\mu_{i}, i=1, \ldots, 4$. Notice that $\mu_{1}$ includes the belief that the car is parked in front of the house with a full fuel tank. (That is, $\vdash_{\mathcal{L}_{e}} \mu_{1} \Rightarrow$ fuel-tank-full $\wedge$ car-parked-outside.) At time 2 the agent makes no observations since she is in her house, so $\mu_{2}=\mu_{1} \diamond$ true $=\mu_{1}$ by U2. At time 3 the agent observes the car outside her house, so $\mu_{3}=\mu_{2} \diamond$ car-parked-outside $=\mu_{1}$, again by U2. Finally, $\mu_{4}=\mu_{3} \diamond \neg$ fuel-tank-full. The observation of $\neg$ fuel-tank-full at time 4 must be explained by some means. In our semantics, the answer is clear. The most plausible runs are these where the car was parked until time 3 , and somewhere between time 3 and 4 some change occurred.

Is this counterintuitive conclusion an artifact of our representation? To some extent it is. This issue cannot be formally addressed within Katsuno and Mendelzon's semantic framework, since that framework does not provide an account of sequences of changes. Moreover, one might argue that within out framework there might be other families of priors that satisfy U1-U8, which will offer alternative explanations of the surprising observation at time 4. Nevertheless, we claim that our semantics captures, in what we believe to be the most straightforward way, the intuition embedded in the Katsuno and Mendelzon's representation. In particular, condition UPD2, which enforces the delay of abnormal events, was needed in order to capture the "pointwise" nature of the update. It would be interesting to know whether there is a natural way of capturing update in our framework that does not suffer from these problems.

Does this way of capturing update semantically ever lead to reasonable results? Of course, that depends on how we interpret "reasonable". We briefly consider one approach here.

In a world $w$, the agent has some beliefs that are described by, say, the formula $\varphi$. These beliefs may or may not be correct (where we say a belief $\varphi$ is correct in a world $w$ if $\varphi$ is true of $w$ ). Suppose something happens and the world changes to $w^{\prime}$. As a result of the agent's observations, she has some new beliefs, described by $\varphi^{\prime}$. Again, there is no reason to believe that $\varphi^{\prime}$ is correct. Indeed, it may be quite unreasonable to expect $\varphi^{\prime}$ to be correct, even if $\varphi$ is correct. Consider the borrowed-car example. Suppose that while the agent was sitting inside the house, the car was, in fact, taken for a ride. Nevertheless, the most reasonable belief for the agent to hold when she observes that the car is still in the parked after she leaves the house is that it was there all along.

The problem here is that the information the agent obtains at times 2 and 3 is insufficient to determine what happened. We cannot expect all the agent's beliefs to be correct at this point. On the other hand, if she does obtain sufficient information about the change and her beliefs were initially correct, then it seems reasonable to expect that her new beliefs will be correct. But what counts as sufficient information?

We say that $\varphi$ provides sufficient information about the change from $w$ to $w^{\prime}$ if there is no world $w^{\prime \prime}$ satisfying $\varphi$ such that $d\left(w, w^{\prime \prime}\right)<d\left(w, w^{\prime}\right)$. In other words, $\varphi$ is sufficient information if, after observing $\varphi$ in world $w$, the agent will consider the real world $\left(w^{\prime}\right)$ one 
of the most likely worlds. Note that this definition is monotonic, in that if $\varphi$ is sufficient information about the change, then so is any formula $\psi$ that implies $\varphi$ (as long as it holds at $\left.w^{\prime}\right)$. Moreover, this definition depends on the agent's distance function $d$. What constitutes sufficient information for one agent might not for another. We would hope that the function $d$ is realistic in the sense that the worlds judged closest according to $d$ really are the most likely to occur.

We can now show that update has the property that if the agent has correct beliefs and receives sufficient information about a change, then she will continue to have correct beliefs.

Theorem 6.4: Let $\mathcal{I} \in \mathcal{C}^{U}$. If the agent's beliefs at $(r, m)$ are correct and $o_{(r, m)}$ provides sufficient information about the change from $r_{e}(m)$ to $r_{e}(m+1)$, then the agent's beliefs at $(r, m+1)$ are correct.

Proof: Straightforward; left to the reader.

As we observed earlier, we cannot expect the agent to always have correct beliefs. Nevertheless, we might hope that if the agent does (eventually) receive sufficiently detailed information, then she should realize that her beliefs were incorrect. But this is precisely what does not happen in the borrowed-car example. Intuitively, once the agent observes that the fuel tank is not full, this should be sufficient information to eliminate the possibility that the car remained in the parking lot. However, it is not. Roughly speaking, this is because update focuses only on the current state of the world, and thus cannot go back and revise beliefs about the past.

The problem here is again due to the fact that belief update is determined only by the agent's belief state and not her epistemic state. Thus, update can only take into account the agent's current beliefs and not other information, such as the sequence of observations that led to these beliefs. In our example, if we limit our attention to beliefs about the car's whereabouts and the fuel tank, then since the agent has the same belief state at time 1 and 3 , she must change her beliefs in the same manner at both times. This implies that the observation the fuel tank is not full at time 4 cannot be sufficient information about the past, since a fuel leak might be the most plausible explanation of missing fuel at time $2 .^{12}$

Our discussion of update shows that update is guaranteed to be safe only in situations where there is always enough information to characterize the change that has occurred. While this may be a plausible assumption in database applications, it seems somewhat less reasonable in $\mathrm{AI}$ examples, particularly in cases involving reasoning about action. ${ }^{13}$

\section{Synthesis}

In previous sections we analyzed belief revision and belief update separately. We provided representation theorems for both notions and discussed issues specific to each notion. In this section, we try to identify some common themes and points of difference.

12. In this example the usual intuition is that, given the observation that the tank is not full, the agent should revise her belief in some manner instead of performing update. This immediately raises the question of how the agent knows what the right belief change operation should be here. We return to this issue below.

13. Similar observations were independently made by Boutilier (1996b), although his representation is quite different from ours. 


\begin{tabular}{|l||c|c|}
\hline Restriction on & Revision & Update \\
\hline \hline Environment changes & $\begin{array}{c}\text { No change } \\
\text { (Static propositions) }\end{array}$ & All possible sequences \\
\hline Initial plausibility & Total preorder & Lexicographic \\
\hline Belief change & Conditioning & Conditioning \\
\hline
\end{tabular}

Table 1: A summary of the restrictions we impose to capture revision and update.

Katsuno and Mendelzon (1991a) focused on the following three differences between AGM revision and KM update:

1. Revision deals with static propositions, while update allows propositions that are not static.

2. Revision and update treat inconsistent belief states differently. Revision allows an agent to "recover" from an inconsistent state after observing a consistent formula. Update dictates that once the agent has inconsistent beliefs, she will continue to have inconsistent beliefs. As we noted above, it seems that revision's ability to recover from an inconsistent belief set leads to several technical anomalies in iterated revision.

3. Revision considers only total preorders, while update allows partial preorders.

Our framework suggests a different approach to categorizing the differences between revision and update (and other approaches to belief change): focusing on the restrictions that have to be added to basic BCSs to obtain systems in $\mathcal{C}^{R}$ and $\mathcal{C}^{U}$, respectively. In particular, we focus on three aspects of a system:

- How does the environment state change?

- How does the agent form her initial beliefs? What regularities appear in the agent's beliefs at the initial state?

- How does the agent change her beliefs?

Table 1 summarizes the answers to these questions for revision and update; it highlights the different restrictions imposed by each. Revision puts a severe restriction on changes of the environment (more precisely, on how we describe the environment in the language) and a rather mild restriction on the agent's prior beliefs (they must form a total preorder). On the other hand, update allows all sequences of environment states, but requires the agent's prior beliefs to have a specific form. These formal properties match the intuitive description of revision and update given in (Alchourrón et al., 1985; Katsuno \& Mendelzon, 1991b). However, the explicit representation of time in our framework allows us to make these intuitions precise. Moreover, our framework makes explicit other assumptions made by revision and update. For example, the lexicographic nature of update is not immediately evident from the presentation in (Katsuno \& Mendelzon, 1991b). 
The key point to notice in this table is that belief change in both revision and update is done by conditioning. This observation, and the naturalness of conditioning as a notion of change, support our claim that conditioning should be adopted as semantic foundations for minimal change.

How significant are the differences between revision and update? We claim that some of these differences are a result of different ways of modeling the same underlying process. Recall that in the introduction we noted that the restriction to static propositions is not such a serious limitation of belief revision, since we can always convert a dynamic proposition to a static one by adding timestamps. More precisely, we can replace a proposition $p$ by a family of propositions $p^{m}$ that stand for " $p$ is true at time $m$ ". This makes it possible to use revision to reason about a changing world. We now show how revision and update can be related under this viewpoint.

To make this discussion precise, we need to introduce some formal definitions. Let $\mathcal{I}=(\mathcal{R}, \pi, \mathcal{P})$ be a BCS. We "statify" $\mathcal{I}$ into a system $\mathcal{I}^{*}=\left(\mathcal{R}^{*}, \pi^{*}, \mathcal{P}^{*}\right)$ by replacing the underlying language with static propositions.

Let $\Phi_{e}^{*}=\left\{p^{m}: p \in \Phi_{e}, m \in N\right\}$ be a set of timestamped propositions and let $\mathcal{L}_{e}^{*}$ be the logical language based on these propositions. We can easily "timestamp" every formula in $\mathcal{L}$. We define timestamp $(\varphi, m)$ recursively as follows. The base case is timestamp $(p, m)=p^{m}$ for $p \in \Phi_{e}$. For standard logical connectives, we simply apply the transformation recursively, for example timestamp $(\varphi \wedge \psi)=\operatorname{timestamp}(\varphi, m) \wedge \operatorname{timestamp}(\psi, m)$.

Next, we define the set of runs in the "statified" system. For each run $r \in \mathcal{R}$, we define a $r^{*}$ in $\mathcal{R}^{*}$ as follows. The environment states in $r^{*}$ are defined to be the whole sequence of environment states in $r$, that is, $r_{e}^{*}(m)=r_{e}$. If $r_{a}(m)=\left\langle o_{(r, 1)}, \ldots, o_{(r, m)}\right\rangle$, we define $r_{a}(m)=\left\langle\operatorname{timestamp}\left(o_{(r, 1)}, 1\right), \ldots, \operatorname{timestamp}\left(o_{(r, m)}, m\right)\right\rangle$. We define the interpretation $\pi^{*}$ in the obvious way: $\pi^{*}\left(r^{*}, m\right)\left(p^{m^{\prime}}\right)=$ true if and only if $\pi\left(r, m^{\prime}\right)(p)=$ true and $\pi^{*}\left(r^{*}, m\right)(\operatorname{learn}(\varphi))=$ true if and only if $o_{\left(r^{*}, m\right)}=\varphi$.

Finally, we need to define the prior plausibility $\mathrm{Pl}_{a}^{*}$. We define this prior to be isomorphic to $\mathrm{Pl}_{a}$ under the transformation $r^{*} \mapsto r$. That is, for each set of runs $R^{*} \subseteq \mathcal{R}^{*}$, we define $\mathrm{Pl}_{a}^{*}\left(R^{*}\right)=\mathrm{Pl}_{a}\left(\left\{r \in \mathcal{R}: r^{*} \in R^{*}\right\}\right)$.

It is clear that the two systems $\mathcal{I}$ and $\mathcal{I}^{*}$ describe the same underlying process. Perhaps the most significant difference is that the environment state in a run of $\mathcal{I}^{*}$ encodes the future of the run. This was necessary so that the environment state could determine the truth of all propositions of the form $p^{m}$, so as to satisfy BCS1. Without this requirement, we could have simply changed $\pi$ and left $\mathcal{R}$ and $\mathcal{P}$ unchanged.

Because different base languages are used in $\mathcal{I}$ and $\mathcal{I}^{*}$, the agent has different beliefs in the two systems. It is easy to show that, for all $\varphi \in \mathcal{L}_{e}$, we have $(\mathcal{I}, r, m) \models B \varphi$ iff $\left(\mathcal{I}^{*}, r^{*}, m\right) \models B$ (timestamp $\left.(\varphi, m)\right)$. However, at $\left(r^{*}, m\right)$ the agent also has beliefs about propositions that describe past and future times. Thus, the set of beliefs of the agent in $\mathcal{I}^{*}$ can be viewed as a superset of her beliefs in $\mathcal{I}$ at the corresponding points.

The following result makes precise the relationship between $\mathcal{I}$ and $\mathcal{I}^{*}$ in terms of the properties we have been considering.

Proposition 7.1: Let $\mathcal{I}$ be a BCS and let $\mathcal{I}^{*}$ the transformed system defined above. Then

- $\mathcal{I}^{*}$ is a BCS, that is, it satisfies BCS1-BCS5.

- $\mathcal{I}^{*}$ satisfies REV1. 
- If $\mathcal{I}$ satisfies UPD马, then $\mathcal{I}^{*}$ satisfies REV3.

- If $\mathcal{I}$ satisfies UPD 4, then $\mathcal{I}^{*}$ satisfies $R E V 4_{4}^{\prime}$.

Proof: Straightforward; left to the reader.

Thus, if $\mathcal{I}$ is a BCS, so is $\mathcal{I}^{*}$. Moreover, if $\mathcal{I} \in \mathcal{C}^{U}$, then $\mathcal{I}^{*}$ satisfies all but two of the requirements for $\mathcal{C}^{R}$. First, $\mathcal{I}^{*}$ does not necessarily satisfy REV2, since the prior of systems in $\mathcal{C}^{U}$ is, in general, not ranked. Second, $\mathcal{I}^{*}$ satisfies REV4', the weaker version of REV4. The reason for this is that runs $\mathcal{I}^{*}$ do not allow all sequences of possible observations. Remember that in the language of $\mathcal{L}_{e}^{*}$, the agent can observe the proposition $p^{2}$ (i.e., that $p$ is true at time 2) at time 1 . However, in the original system, the agent only observes properties of the current time. Thus, $o_{\left(r^{*}, m\right)}$ involves only propositions that deal with time $m$.

Neither of these shortcomings is serious. First, variants of AGM revision that involve partial orders were discussed in the literature (Katsuno \& Mendelzon, 1991b; Rott, 1992). It is fairly straightforward to show that these can captured in our systems using BCSs that satisfy REV1, REV3, and REV4. Second, it is easy to add to $\mathcal{I}^{*}$ runs so as to get a system that satisfies REV4. Moreover, we can do this is a way that does not change the agent's beliefs for sequences of observations that can be observed in $\mathcal{I}$. Thus, the "statified" version of a system in $\mathcal{C}^{U}$ displays behavior much in the spirit of belief revision.

This result may seem somewhat surprising in light of the significant differences between the AGM postulates and KM postulates. In part, it shows how much is bound up in our choice of language. (Recall that similar issues arose in Example 5.1.) This highlights the sensitivity of the postulate approach to the modeling assumptions we make. Unfortunately, these modeling assumptions are rarely discussed in the belief change literature. (See (Friedman \& Halpern, 1998a) for a more detailed discussion of this point.)

Table 1 emphasizes that, despite the well-known differences between revision and update, they can be viewed as sharing one very important feature: they both use conditioning to do belief change. Thus, we have a common mechanism both for understanding and extending them. To a certain extent, our results show that revision is more general than update, in the sense that we can view the statified version of any system in $\mathcal{C}^{U}$ as performing revision (possibly with unranked prior) over runs.

\section{Extensions}

In the preceding sections, we introduced several assumptions that were needed to capture revision and update. Of course, there are other ways of capturing these notions that require somewhat different assumptions. Nevertheless, these assumptions give insight into the underlying choices made, either explicitly or implicitly, in the definition of revision and update. In addition, thinking in terms of such restrictions makes it straightforward to extend the intuitions of revision and update beyond the context where they were originally applied. In this section, we consider a number of such extensions, to illustrate our point. 


\subsection{Knowledge}

In many domains of interest, the agent knows that some sequences of observations are impossible. We already saw in the circuit-diagnosis problem that observing failures was impossible. In the context of update, we know that we cannot observe a person die and then be alive, despite the fact that both being dead and being alive are consistent states.

We can easily maintain what we regard as the defining properties of revision and update, as discussed in the previous section: no change in the environment state and a ranked prior in the case of revision, and a lexicographic prior in the case of update, with belief change proceeding by conditioning in both cases. We simply drop REV3 and replace REV4 by REV4' (resp., drop UPD3 and replace UPD4 and UPD4'). We remark that this change affects the postulates. For example, consider update. Suppose that the agent considers the possibility that Mr. Bond is dead. If she then observes Mr. Bond alive and well then, according to update, she must account for the new observation by some change from the worlds she previously considered possible. However, there is no transition from worlds in which Mr. Bond is dead that can account for the new observation. Thus, once the agent knows that certain transitions are impossible, some observations (e.g., observing that Mr. Bond is alive) require her to remove from consideration some of the worlds that she previously considered possible. As a consequence, postulate U8 does not hold, since the agent's new beliefs are not determined by a pointwise update at each of the worlds she previously considered possible. (Boutilier (1998) uses a related semantic framework to draw similar conclusions in his analysis of update.)

\subsection{Language of Beliefs}

In our analysis of revision and update, we focused on the agent's beliefs about the current state of the environment. Often we are also interested in how the agent changes her beliefs about other types of statements, such as beliefs about future states of the environment, beliefs about other agents' beliefs, and introspective beliefs about her own beliefs. Again, it is straightforward in our framework to deal with an enriched language that lets us express such statements. For example, in (Friedman \& Halpern, 1994) we examine Ramsey conditionals. These are formulas of the form $\varphi>\psi$, which can be read as saying "after learning $\varphi$, the agent believes $\psi$ ". This formula can be expressed as $\operatorname{learn}(\varphi) \Rightarrow B \psi$ in the language $\mathcal{L}^{K P T}$. As is well known, if belief sets include Ramsey conditionals (and not just propositional formulas), then the AGM postulates become inconsistent (at least, provided we have at least three mutually exclusive consistent formulas in the language) (Gärdenfors, 1986). Similar inconsistency results arise when one tries to add other forms of introspective beliefs (Fuhrmann, 1989). In our setting, it is easy to see why the problem arises. Even if we allow belief sets to include nonpropositional formulas, it still seems quite clear that we want to distinguish the propositional formulas from formulas that talk explicitly about an agent's beliefs. For example, it is not clear that we should allow an observation of a formula such as $\varphi>\psi$. What would it mean to observe such a formula? It clearly seems quite different from observing a propositional formula. Nor does it make sense to extend an assumption such as REV1 to arbitrary formulas. While it may be reasonable to restrict to static propositions if we are viewing these as making statements about a relatively stable 
environment, it seems far less reasonable to assume that formulas that talk about an agent's beliefs will be static, especially when we are trying to model belief change!

Of course, if we allow only propositional formulas to be learned (or observed), and restrict REV1 to propositional formulas, then it is easy to see that all of our results still hold, even if the full language is quite rich; we avoid the triviality result completely.

\subsection{Observations}

One of the strongest assumptions made by revision and update involves the treatment of observations. This assumption seems unreasonable in most domains. REV4 and UPD4 essentially assume that the observation that the agent makes is chosen randomly among all formulas consistent with the current state of the world. Suppose that $\varphi$ says that the agent is outdoors, $\psi$ says that the agent is in the basement, and $o_{1}$ says that the basement light is on. We may well have $\mathrm{Pl}_{a}\left(\mathcal{R}\left[\varphi \wedge o_{1}\right]\right)>\mathrm{Pl}_{a}\left(\mathcal{R}\left[\psi \wedge o_{1}\right]\right)$. For example, the agent may hardly ever go to the basement and frequently go outdoors, but her children may often leave the basement light on. Nevertheless, we may also have $\mathrm{Pl}_{a}\left(\mathcal{R}\left[\varphi ; o_{1}\right]\right)<\mathrm{Pl}_{a}\left(\mathcal{R}\left[\psi ; o_{1}\right]\right)$, contradicting REV4. Indeed, it may well be impossible for the agent to observe that the basement light is on when she is outdoors, so that $\mathrm{Pl}_{a}\left(\mathcal{R}\left[\varphi ; o_{1}\right]\right)=\perp$, but this is not permitted according to REV4 or UPD4.

In many domains it is useful to reason about hidden quantities that simply cannot be observed. For example, the event that component $c_{i}$ is faulty in Example 5.1 is a basic event in our description of the problem, yet it cannot be observed. Similarly, the event where a patient has a disease $\mathrm{X}$ or the opponent is planning to capture the queen are useful in reasoning about medical diagnosis and game strategy, yet are not directly observable in practice. Thus, the requirement that all formulas in the language can be observed seems quite unnatural. We note that explicitly modeling sensory input is a standard practice in control theory and stochastic processes (e.g., in hidden Markov chains). In these fields, one models the probability of an observation in various situations. Making an observation increases the probability of situations where that observation is likely to be observation and decreases the probability of situations where it is unlikely. Again, it is straightforward to consider a more detailed model of the observation process in our framework; see (Friedman, 1997, Chapter 6) and (Boutilier et al., 1998).

\subsection{Actions}

Our definition of belief change systems essentially assumes that the agent is passive. The situation is more complex when the agent can influence the environment. The agent's choice of action interacts with her beliefs. It is clear that after performing an action, the agent should change her beliefs. ${ }^{14}$ Moreover, the information content of observations depends on the action the agent has just performed. For example, the agent might consider hearing a loud noise to be surprising. However, it would be expected after the agent pulls the trigger of her gun.

14. Indeed, an alternative interpretation of the update postulates is that they describe how the agent should update her beliefs after doing the action "achieve $\varphi$ " (Goldszmidt \& Pearl, 1996; del Val \& Shoham, 1992, 1993). However, as these works show, the update postulates are problematic under this interpretation. 


\subsection{Summary}

This list of possible extensions is clearly not exhaustive; there are many others that we may want to consider. Nevertheless, these are extensions that seem to be of interest. The main points we want to make here are (1) it is easy to accommodate these extensions in our framework while still maintaining the main characteristics of revision and update, and (2) it is difficult to deal with such extensions if we focus on postulates.

\section{Conclusion}

We have shown how the framework introduced in (Friedman \& Halpern, 1997) can be used to capture belief revision and update. Modeling revision and update in the framework also gives us a great deal of further insight into their properties, and emphasizes the role of conditioning as a way of capturing minimal change.

Of course, revision and update are but two points in a wide spectrum of possible types of belief change. Our ultimate goal is to use this framework to understand the whole spectrum better and to help us design belief change operations that overcome some of the difficulties we have observed with revision and update. In particular, we want belief change operations that can handle dynamic propositions, while still being able to revise information about the past.

Our framework suggests how to construct such belief change operations. In this framework, belief change operations can be determined by choosing a plausibility measure that captures the agent's preferences among sequences of worlds. This is the agent's prior pla1sibility, and captures her initial beliefs about the relative likelihood of runs. As the agent receives information, she changes her beliefs using conditioning. In this paper we show that revision and update correspond to two specific families of priors. Clearly, however, there are prior plausibilities that, when conditioned on a surprising observation, allow the agent to revise some earlier beliefs and to assume that some change has occurred. One obvious problem is that, even if there are only two possible states, there are uncountably many possible runs. How can an agent describe a prior plausibility over such a complex space?

One approach to doing this is based on intuition from the probabilistic settings. In these settings, the standard solution to this problem is to assume that state transitions are independent of when they occur, that is, that the probability of the system going from state $s$ to state $s^{\prime}$ is independent of the sequence of transitions that brought the system to state s. This Markov assumption significantly reduces the complexity of the problem. All that is necessary is to describe the probability of state transitions. In (Friedman \& Halpern, 1996; Friedman, 1997) we define a notion of plausibilistic independence, and show how to describe priors that satisfy the Markov assumption and the consequences for belief change. See also (Boutilier, 1998; Boutilier et al., 1998) for recent proposals along these lines.

Whether or not this particular approach turns out to be a useful one, it is clear that these are the types of questions we should be asking. As these works show, our framework provides a useful basis for answering them.

Finally, we note that our approach is quite different from the traditional approach to belief change (Alchourrón et al., 1985; Gärdenfors, 1988; Katsuno \& Mendelzon, 1991a). Traditionally, belief change was viewed as an abstract process. Our framework, on the other hand, models the agent and the environment she is situated in, and how both change in time. 
This allows us to model concrete agents in concrete settings (for example, diagnostic systems are analyzed in (Friedman \& Halpern, 1997) and throughout this paper), and to reason about the beliefs and knowledge of such agents. We can then investigate what plausibility ordering induces beliefs that match our intuitions. By gaining a better understanding of such concrete situations, we can better investigate more abstract notions of belief change. More generally, we believe that, when studying belief change, it is important to specify the underlying ontology: that is, exactly what scenario underlies the belief-change process. We have specified one such scenario here. While others are certainly possible, we view it as a defect in the literature on belief change that the underlying scenario is so rarely discussed. The framework we have introduced here provides a way of making formal what the scenario is. (See (Friedman \& Halpern, 1998a) for further discussion of this issue.)

\section{Acknowledgments}

The authors are grateful to Craig Boutilier, Ronen Brafman, Adnan Darwiche, Moises Goldszmidt, Adam Grove, Alberto Mendelzon, Alvaro del Val, and particularly Daphne Koller and Moshe Vardi, for comments on drafts of this paper and useful discussions relating to this work. Some of this work was done while both authors were at the IBM Almaden Research Center. The first author was also at Stanford while much of the work was done. IBM and Stanford's support are gratefully acknowledged. The work was also supported in part by the Air Force Office of Scientific Research (AFSC), under Contract F49620-91-C-0080 and grant F94620-96-1-0323 and by NSF under grants IRI-95-03109 and IRI-96-25901. The first author was also supported in part by an IBM Graduate Fellowship and by Rockwell Science Center. A preliminary version of this paper appears in J. Doyle, E. Sandewall, and P. Torasso (Eds.), Principles of Knowledge Representation and Reasoning: Proc. Fourth International Conference, 1994, pp. 190-201, under the title "A knowledge-based framework for belief change, Part II: revision and update."

\section{Appendix A. Proofs}

\section{A.1 Proofs for Section 5}

We start with the proof of Theorems 5.2 and 5.3. To do this, we need some preliminary definitions and lemmas. Figure 1 shows the general outline of the intermediate representations we use in these proofs. Roughly speaking, we show how to map from a revision operator $O$ and a consistent belief set $K$ to a ranking, and similarly how to map from a ranking to an AGM revision operator. These rankings correspond, in a direct way, to priors in systems in $\mathcal{C}^{R}$, and thus have close connection to the beliefs of the agent in various states.

These mapping between AGM revision operators and rankings are related to the representation theorems of Boutilier (1994b), Grove (1988), and Katsuno and Mendelzon (1991a). However, the exact details of our representations are different than those of Boutilier, Grove, and Katsuno and Mendelzon. Thus, for completeness we provide the full proofs here.

We start with the mapping from revision operator applied to a specific belief set to a ranking. As an intermediate step we construct a set of defaults as follows. We then will use the results from (Friedman \& Halpern, 1998b) to construct a ranked plausibility structure that satisfies these defaults. 


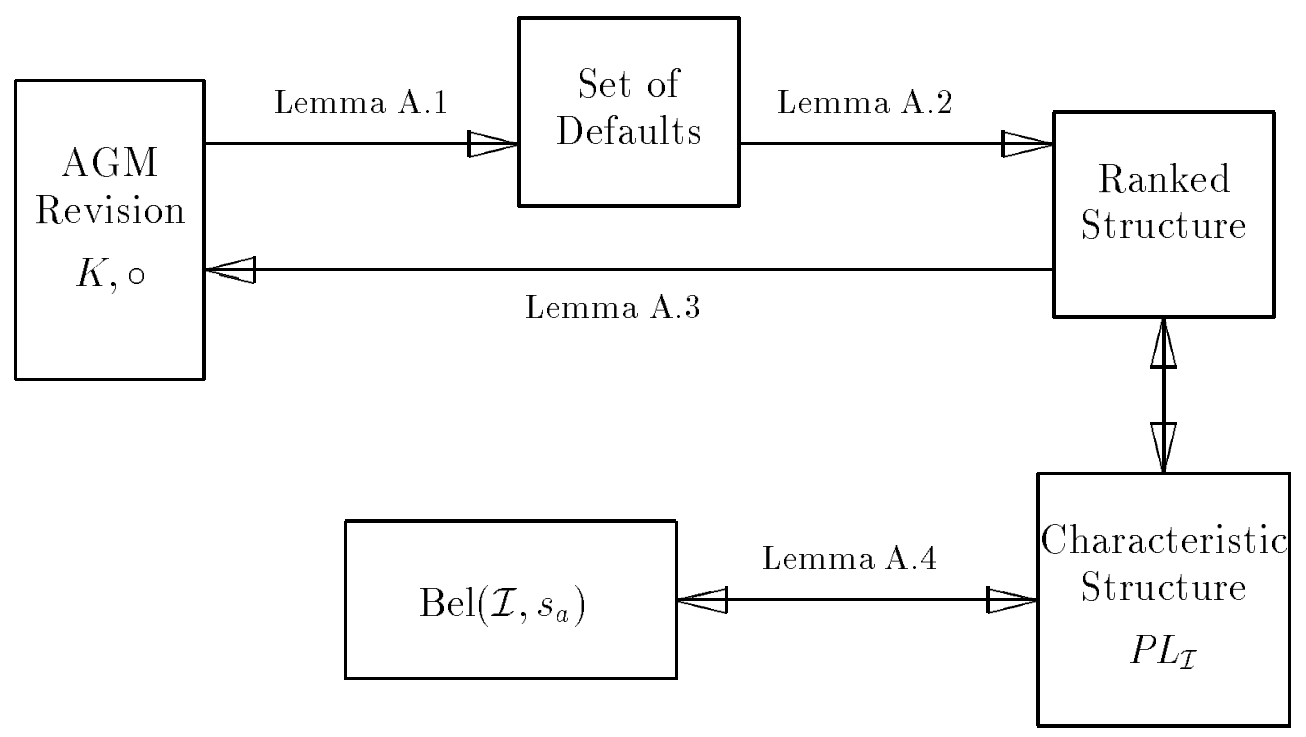

Figure 1: Schematic description of the entities and lemmas involved in the proof of Theorems 5.2 and 5.3 .

Lemma A.1: Let $\circ$ be an $A G M$ revision operator, let $K \subseteq \mathcal{L}_{e}$ be a consistent belief set, and let

$$
\Delta_{(\circ, K)}=\left\{\varphi \rightarrow \psi: \varphi, \psi \in \mathcal{L}_{e}, \psi \in K \circ \varphi\right\}
$$

Then the following is true:

(a) $\Delta_{(o, K)}$ is closed under the rules of system $\mathbf{P}$,

(b) $\varphi \rightarrow$ false $\notin \Delta_{(\circ, K)}$ for all consistent $\varphi \in \mathcal{L}_{e}$, and

(c) $\Delta_{(\circ, K)}$ satisfies rational monotonicity; that is, if $\varphi \rightarrow \psi \in \Delta_{(\circ, K)}$ and $\varphi \rightarrow \neg \xi \notin \Delta_{(\circ, K)}$, then $\varphi \wedge \xi \rightarrow \psi \in \Delta_{(\circ, K)}$.

Proof: We start with part (a):

LLE Assume that $\vdash_{\mathcal{L}_{e}} \varphi \equiv \varphi^{\prime}$ and that $\varphi \rightarrow \psi \in \Delta_{(\circ, K)}$. Thus, $\psi \in K \circ \varphi$. From R5, it follows that $\psi \in K \circ \varphi^{\prime}$, and thus $\varphi^{\prime} \rightarrow \psi \in \Delta_{(0, K)}$.

RW Assume that $\vdash_{\mathcal{L}_{e}} \psi \Rightarrow \psi^{\prime}$ and that $\varphi \rightarrow \psi \in \Delta_{(\circ, K)}$. Thus, $\psi \in K \circ \varphi$. Since $K \circ \varphi$ is a belief set, it is closed under logical consequence. In particular, $\psi^{\prime} \in K \circ \varphi$, and hence $\varphi \rightarrow \psi^{\prime} \in \Delta_{(\circ, K)}$.

REF By R2, $\varphi \in K \circ \varphi$, and thus, $\varphi \rightarrow \varphi \in \Delta_{(\circ, K)}$.

AND Assume that $\varphi \rightarrow \psi_{1}, \varphi \rightarrow \psi_{2} \in \Delta_{(\circ, K)}$. Thus, $\psi_{1}, \psi_{2} \in K \circ \varphi$. Since $K \circ \varphi$ is a belief set, $\psi_{1} \wedge \psi_{2} \in K \circ \varphi$. Thus, $\varphi \rightarrow \psi_{1} \wedge \psi_{2} \in \Delta_{(\circ, K)}$.

OR Assume that $\varphi_{1} \rightarrow \psi, \varphi_{2} \rightarrow \psi \in \Delta_{(\circ, K)}$. There are two cases. If $K \circ\left(\varphi_{1} \vee \varphi_{2}\right)$ is inconsistent, then $\psi \in K \circ\left(\varphi_{1} \vee \varphi_{2}\right)$ and thus $\varphi_{1} \vee \varphi_{2} \rightarrow \psi \in \Delta_{(\circ, K)}$. If $K \circ\left(\varphi_{1} \vee \varphi_{2}\right)$ is 
consistent, then, by $R 2, \varphi_{1} \vee \varphi_{2} \in K \circ\left(\varphi_{1} \vee \varphi_{2}\right)$. Thus, we cannot have both $\neg \varphi_{1}$ and $\neg \varphi_{2}$ in $K \circ\left(\varphi_{1} \vee \varphi_{2}\right)$. Without loss of generality, assume that $\neg \varphi_{1} \notin K \circ\left(\varphi_{1} \vee \varphi_{2}\right)$. Using R7 and R8, we get that $K \circ\left(\left(\varphi_{1} \vee \varphi_{2}\right) \wedge \varphi_{1}\right)=C l\left(K \circ\left(\varphi_{1} \vee \varphi_{2}\right) \cup\left\{\varphi_{1}\right\}\right)$. Using R6, we get that $K \circ\left(\left(\varphi_{1} \vee \varphi_{2}\right) \wedge \varphi_{1}\right)=K \circ \varphi_{1}$. Thus, we conclude that $K \circ \varphi_{1}=C l\left(K \circ\left(\varphi_{1} \vee \varphi_{2}\right) \cup\left\{\varphi_{1}\right\}\right)$. Since $\varphi_{1} \rightarrow \psi \in \Delta_{(\circ, K)}$, we have that $\psi \in K \circ \varphi_{1}$. Thus, we get that $\varphi_{1} \Rightarrow \psi \in K \circ\left(\varphi_{1} \vee \varphi_{2}\right)$. If $\neg \varphi_{2} \notin K \circ\left(\varphi_{1} \vee \varphi_{2}\right)$, by similar arguments we get that $\varphi_{2} \Rightarrow \psi \in K \circ\left(\varphi_{1} \vee \varphi_{2}\right)$. This implies that $\left(\varphi_{1} \vee \varphi_{2}\right) \Rightarrow \psi \in K \circ\left(\varphi_{1} \vee \varphi_{2}\right)$, and thus $\psi \in K \circ\left(\varphi_{1} \vee \varphi_{2}\right)$. On the other hand, if $\neg \varphi_{2} \in K \circ\left(\varphi_{1} \vee \varphi_{2}\right)$, then, since $\varphi_{1} \vee \varphi_{2} \in K \circ\left(\varphi_{1} \vee \varphi_{2}\right)$, we get that $\varphi_{1} \in K \circ\left(\varphi_{1} \vee \varphi_{2}\right)$, and thus $\psi \in K \circ\left(\varphi_{1} \vee \varphi_{2}\right)$.

CM Assume that $\varphi \rightarrow \psi_{1}, \varphi \rightarrow \psi_{2} \in \Delta_{(\circ, K)}$. If $K \circ \varphi$ is inconsistent, then using R5 we get that $\varphi$ is inconsistent. Thus, $\varphi \wedge \psi_{1}$ is inconsistent, so $\psi_{2} \in K \circ\left(\varphi \wedge \psi_{1}\right)$. Now assume that $K \circ \varphi$ is consistent. Since $\varphi \rightarrow \psi_{1}$, we have that $\psi_{1} \in K \circ \varphi$. Since $K \circ \varphi$ is consistent, we get that $\neg \psi_{1} \notin K \circ \varphi$. Applying $\mathrm{R} 8$, we get that $K \circ \varphi \subseteq K \circ\left(\varphi \wedge \psi_{1}\right)$. Since $\varphi \rightarrow \psi_{2} \in \Delta_{(\circ, K)}$, we have that $\psi_{2} \in K \circ \varphi$. Thus, $\psi_{2} \in K \circ\left(\varphi \wedge \psi_{1}\right)$. This implies that $\left(\varphi \wedge \psi_{1}\right) \rightarrow \psi_{2} \in \Delta_{(\circ, K)}$.

We now prove part (b). Let $\varphi \in \mathcal{L}_{e}$ be a consistent formula. Then, using R5, we get that $K \circ \varphi$ is consistent. Thus, $\varphi \rightarrow$ false $\notin \Delta_{(o, K)}$.

Finally we prove part (c). Assume that $\varphi \rightarrow \psi \in \Delta_{(0, K)}$, and $\varphi \wedge \xi \rightarrow \psi \notin \Delta_{(0, K)}$. Since $\varphi \rightarrow \psi \in \Delta_{(\circ, K)}$, we have that $\psi \in K \circ \varphi$. Now if $\neg \xi \notin K \circ \varphi$, then, using R8, we have that $C l(K \circ \varphi \cup\{\xi\}) \subseteq K \circ(\varphi \wedge \xi)$. This implies that $\psi \in K \circ(\varphi \wedge \xi)$. However, since we assumed that $\varphi \wedge \xi \rightarrow \psi \notin \Delta_{(\circ, K)}$, we have that $\psi \notin K \circ(\varphi \wedge \xi)$; thus, we get a contradiction. We conclude that $\neg \xi \in K \circ \varphi$. Thus, $\varphi \rightarrow \neg \xi \in \Delta_{(\circ, K)}$.

We now use this result to show that there exists a plausibility structure that corresponds to $\circ$ applied to $K$.

Lemma A.2: Let $\circ$ be an $A G M$ revision operator, and let $K \subseteq \mathcal{L}_{e}$ be a consistent belief set. Then there is a plausibility structure $P L=(W, \mathrm{Pl}, \pi)$ such that $\mathrm{Pl}$ is ranked, $P L \models \varphi \rightarrow \psi$ if and only if $\psi \in K \circ \varphi$, and $\mathrm{Pl}(\llbracket \varphi \rrbracket)>\perp$ for all $\vdash_{\mathcal{L}_{e}}$-consistent formulas $\varphi \in \mathcal{L}_{e}$.

Proof: We use the basic techniques described in the proof of (Friedman \& Halpern, 1998b, Theorem 8.2). Let $\Delta_{(\circ, K)}$ be the set of defaults defined by Lemma A.1. We now construct a plausibility space $P L^{\prime}=\left(W, \mathrm{Pl}^{\prime}, \pi\right)$ such that $P L^{\prime} \models \varphi \rightarrow \psi$ if and only if $\varphi \rightarrow \psi \in \Delta_{(\circ, K)}$. We define $P L^{\prime}$ as follows:

- $W=\left\{w_{V}: V \subseteq \mathcal{L}_{e}\right.$ is a maximal $\vdash_{\mathcal{L}_{e}}$-consistent set $\}$,

- $\pi\left(w_{V}\right)(p)=$ true if $p \in V$, and

- $\mathrm{Pl}^{\prime}(\llbracket \varphi \rrbracket) \geq \mathrm{Pl}^{\prime}(\llbracket \psi \rrbracket)$ if and only if $(\varphi \vee \psi) \rightarrow \varphi \in \Delta_{(\circ, K)}$.

Using (Friedman \& Halpern, 1998b, Lemma 4.1), we get that $P L^{\prime} \models \varphi \rightarrow \psi$ if and only if $\varphi \rightarrow \psi \in \Delta_{(o, K)}$. From Lemma A.1 (c) and and results of (Friedman \& Halpern, 1998b), it follows that there is a ranked plausibility measure $\mathrm{Pl}$ that is default-isomorphic to $\mathrm{Pl}^{\prime}$, that is $(W, \mathrm{P} l, \pi)$ satisfies precisely the same defaults as $\left(W, \mathrm{Pl} l^{\prime}, \pi\right)$. Let $P L=(W, \mathrm{P} l, \pi)$.

Since $P L$ is default-isomorphic to $P L^{\prime}$, we have that $P L \models \varphi \rightarrow \psi$ if and only if $\varphi \rightarrow \psi \epsilon$ $\Delta_{(o, K)}$. Moreover, using Lemma A.1, we have that $\varphi \rightarrow \psi \in \Delta_{(\circ, K)}$ if and only if $\psi \in K \circ \varphi$. Thus, $P L \models \varphi \rightarrow \psi$ if and only if $\psi \in K \circ \varphi$. Finally, let $\varphi$ be a $\vdash_{\mathcal{L}_{e}}$-consistent formula. From 
Lemma A.1 (b), we get that $\varphi \rightarrow$ false $\notin \Delta_{(o, K)}$. Since $\Delta_{(o, K)}$ is closed under the rules of system $\mathbf{P}$, we conclude that $(\varphi \vee$ false $) \rightarrow$ false $\notin \Delta_{(o, K)}$. Thus, $\mathrm{Pl}^{\prime}(\llbracket \varphi \rrbracket) \leq \perp=\mathrm{Pl}^{\prime}(\llbracket$ false $\rrbracket)$, and thus $\mathrm{Pl}^{\prime}(\llbracket \varphi \rrbracket)>\perp$. Since $\mathrm{Pl}$ is default-isomorphic to $\mathrm{Pl}^{\prime}$, we conclude that $\mathrm{P}(\llbracket \varphi \rrbracket)>\perp$.

We now prove the converse to Lemma A.2.

Lemma A.3: Let $P L=(W, \mathrm{Pl}, \pi)$ be a ranked plausibility structure such that $\pi(w)$ is $\vdash_{\mathcal{L}_{e}}$ consistent for all worlds $w$, and $P L \forall \forall \rightarrow$ false for all $\vdash_{\mathcal{L}_{e}}$-consistent formulas $\varphi \in \mathcal{L}_{e}$; let $K=\left\{\varphi \in \mathcal{L}_{e}: P L \models\right.$ true $\left.\rightarrow \varphi\right\}$. Then there is an AGM revision operator $\circ$ such that $\psi \in K \circ \varphi$ if and only if $P L \models \varphi \rightarrow \psi$.

Proof: Let $\circ$ be some belief change operation such that $K \circ \varphi=\{\psi: P L \models \varphi \rightarrow \psi\}$. Since this requirement constrains only the result of applying $\circ$ to $K$, we can assume without loss of generality that o satisfies the AGM postulates when applied to belief sets other than $K$. Thus, we need prove only that o satisfies the AGM postulates for revision applied to $K$. (Note that the proofs for R3 and R4 follow from the proofs for R7 and R8, respectively.)

R1 Since $P L$ is qualitative, we have that $\{\psi: P L \models \varphi \rightarrow \psi\}$ is a belief set, that is, closed under logical consequences.

R2 Axiom C1 implies that $P L \models \varphi \rightarrow \varphi$. Thus, $\varphi \in K \circ \varphi$.

R5 By our assumptions, if $\varphi$ is $\vdash_{\mathcal{L}_{e}}$-consistent, then $\mathrm{P} 1(\llbracket \varphi \rrbracket)>\perp$, and thus $P L \forall \varphi \rightarrow$ false.

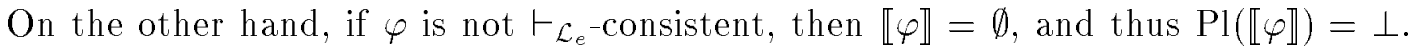
We conclude that $\mathrm{P}(\llbracket \varphi \rrbracket)=\perp$ if and only if $\vdash_{\mathcal{L}_{e}} \neg \varphi$. This implies that $P L \models \varphi \rightarrow$ false if and only if $\vdash_{\mathcal{L}_{e}} \neg \varphi$. Thus, $K \circ \varphi=C l($ false $)$ if and only if $\vdash_{\mathcal{L}_{e}} \neg \varphi$.

R6 Assume that $\vdash_{\mathcal{L}_{e}} \varphi \Leftrightarrow \varphi^{\prime}$. Then, by our assumption, $\pi(w)(\varphi)=\pi(w)\left(\varphi^{\prime}\right)$. Thus, $\llbracket \varphi \wedge \psi \rrbracket=\llbracket \varphi^{\prime} \wedge \psi \rrbracket$ for all formulas $\psi \in \mathcal{L}_{e}$. We conclude that $P L \models \varphi \rightarrow \psi$ if and only if $P L \models \varphi^{\prime} \rightarrow \psi$. This implies that $K \circ \varphi=K \circ \varphi^{\prime}$.

R7 There are two cases: either $\operatorname{Pl}(\llbracket \varphi \wedge \psi \rrbracket)=\perp$ or $\operatorname{Pl}(\llbracket \varphi \wedge \psi \rrbracket)>\perp$. If $\operatorname{Pl}(\llbracket \varphi \wedge \psi \rrbracket)=$ $\perp$, then $\varphi \wedge \psi$ is inconsistent. According to $R 2$, we have that $\varphi \in K \circ \varphi$. Thus, $\varphi \wedge \psi \in C l(K \circ \varphi \cup\{\psi\})$. This implies that $C l(K \circ \varphi \cup\{\psi\})$ contains false, and thus $K \circ(\varphi \wedge \psi) \subseteq C l(K \circ \varphi \cup\{\psi\})$. If $\operatorname{Pl}(\llbracket \varphi \wedge \psi \rrbracket)>\perp$, let $\xi \in K \circ(\varphi \wedge \psi)$. We now show that $\xi \in C l(K \circ \varphi \cup\{\psi\})$. This will show that $K \circ(\varphi \wedge \psi) \subseteq C l(K \circ \varphi \cup\{\psi\})$. Since $\xi \in K \circ(\varphi \wedge \psi)$, we get that $P L \models(\varphi \wedge \psi) \rightarrow \xi$. Since $\operatorname{Pl}(\llbracket \varphi \wedge \psi \rrbracket)>\perp$, we get that $\mathrm{P} 1(\llbracket \varphi \wedge \psi \wedge \xi \rrbracket)>\operatorname{Pl}(\llbracket \varphi \wedge \psi \wedge \neg \xi \rrbracket)$. Then we have that $\mathrm{Pl}(\llbracket \varphi \wedge(\psi \Rightarrow \xi) \rrbracket)>\operatorname{Pl}(\llbracket \varphi \wedge \neg(\psi \Rightarrow$ $\xi)) \rrbracket)$, since $(\varphi \wedge \psi \wedge \xi) \Rightarrow(\varphi \wedge(\psi \Rightarrow \xi))$ and $(\varphi \wedge \neg(\psi \Rightarrow \xi)) \Rightarrow(\varphi \wedge \psi \wedge \neg \xi)$. This also implies that $\mathrm{Pl}(\llbracket \varphi \rrbracket)>\perp$. Thus, $P L \models \varphi \rightarrow(\psi \Rightarrow \xi)$. So, $(\psi \Rightarrow \xi) \in K \circ \varphi$, and thus $\xi \in C l(K \circ \varphi \cup\{\psi\})$.

R8 Assume that $\neg \psi \notin K \circ \varphi$. Let $\xi \in C l(K \circ \varphi \cup\{\psi\})$. We now show that $\xi \in K \circ(\varphi \wedge \psi)$. This will show that $C l(K \circ \varphi \cup\{\psi\}) \subseteq K \circ(\varphi \wedge \psi)$. Let $A=\llbracket \varphi \wedge \neg \psi \rrbracket, B=\llbracket \varphi \wedge \psi \wedge \xi \rrbracket$, and $C=\llbracket \varphi \wedge \psi \wedge \neg \xi \rrbracket$. It is easy to verify that these sets are pairwise disjoint. Since $\varphi \wedge(\psi \Rightarrow \xi) \equiv(\varphi \wedge \neg \psi) \vee(\varphi \wedge \psi \wedge \xi)$ and $(\varphi \wedge \neg(\psi \Rightarrow \xi)) \equiv(\varphi \wedge \psi \wedge \neg \xi)$, we conclude that $\llbracket \varphi \wedge(\psi \Rightarrow \xi) \rrbracket=A \cup B$, and $\llbracket \varphi \wedge \neg(\psi \Rightarrow \xi) \rrbracket=C$. Since $\xi \in C l(K \circ \varphi \cup\{\psi\})$, we have that $(\psi \Rightarrow \xi) \in K \circ \varphi$. This means that $P L \models \varphi \rightarrow(\psi \Rightarrow \xi)$. Thus, either 
$\mathrm{Pl}(\llbracket \varphi \rrbracket)=\perp$ or $\mathrm{Pl}(A \cup B)>\mathrm{Pl}(C)$. If $\mathrm{Pl}(\llbracket \varphi \rrbracket)=\perp$, then according to A1, we get that $\mathrm{P} 1(\llbracket \varphi \wedge \psi \rrbracket)=\perp$. Thus, $P L \models(\varphi \wedge \psi) \rightarrow \xi$ vacuously, and $\xi \in K \circ(\varphi \wedge \psi)$ as desired.

Now assume that $\mathrm{Pl}(A \cup B)>\mathrm{Pl}(C)$. Since $\mathrm{Pl}$ is ranked, it satisfies $\mathrm{A} 4^{\prime}$ and $\mathrm{A} 5^{\prime}$. According to $\mathrm{A}^{\prime}$, we get that either $\mathrm{Pl}(A)>\mathrm{Pl}(C)$ or $\mathrm{Pl}(B)>\mathrm{Pl}(C)$. Assume that $\mathrm{Pl}(A)>\mathrm{Pl}(C)$ and $\mathrm{Pl}(B) \ngtr \mathrm{Pl}(C)$. Then, using $\mathrm{A} 44^{\prime}$, we get that $\mathrm{Pl}(A)>\mathrm{Pl}(B)$. Applying $\mathrm{A} 2$, we get that $\mathrm{Pl}(A)>\mathrm{Pl}(B \cup C)$. However since $A=\llbracket \varphi \wedge \neg \psi \rrbracket$ and $B \cup C=\llbracket \varphi \wedge \psi \rrbracket$, this implies that $\neg \psi \in K \circ \varphi$, which contradicts our assumption. Thus, we conclude that $\mathrm{Pl}(B)>\mathrm{Pl}(C)$. Since $B=\llbracket \varphi \wedge \psi \wedge \xi \rrbracket$ and $C=\llbracket \varphi \wedge \psi \wedge \neg \xi \rrbracket$, we get that $P L \models(\varphi \wedge \psi) \rightarrow \xi$, and thus $\xi \in K \circ(\varphi \wedge \psi)$.

R3 and R4 Our definition of $\circ$ implies that $K \circ$ true $=K$. According to R6, we have that $K \circ($ true $\wedge \varphi)=K \circ \varphi$. Combining these two facts, we get that R3 and R4 are special cases of $\mathrm{R} 7$ and $\mathrm{R} 8$, respectively.

These results show how to map between ranked plausibility structures and AGM revision operators. We now relate systems in $\mathcal{C}^{R}$ and ranked plausibility structures. Let $\mathcal{I}=$ $(\mathcal{R}, \pi, \mathcal{P}) \in \mathcal{C}^{R}$. Recall that $\mathrm{REV} 2$ requires that the prior of $\mathcal{I}$ be a ranking. Thus, we can construct a ranked plausibility structure where worlds are runs in $\mathcal{R}$. We define the characteristic structure of $\mathcal{I}$ to be $P L_{\mathcal{I}}=\left(\mathcal{R}, \mathrm{Pl}_{a}, \pi_{\mathrm{Pl}_{\mathcal{I}}}\right)$, where $\mathrm{Pl}_{a}$ is the agent's prior over runs and $\pi_{\mathrm{Pl}_{\mathcal{I}}}(r)(p)=\pi(r, 0)(p)$ for all $p \in \Phi_{e}$. Note that $\llbracket \varphi \rrbracket_{P L_{\mathcal{I}}}=\mathcal{R}[\varphi]$.

We now use $P L_{\mathcal{I}}$ to describe the beliefs of the agent in each local state.

Lemma A.4: Let $\mathcal{I} \in \mathcal{C}^{R}$ and let $s_{a}=\left\langle o_{1}, \ldots, o_{m}\right\rangle$. Then $\varphi \in \operatorname{Bel}\left(\mathcal{I}, s_{a}\right)$ if and only if $P L_{\mathcal{I}} \models\left(\bigwedge_{i=1}^{m} o_{i}\right) \rightarrow \varphi$. (By convention, if $m=0$, we take $\left(\bigwedge_{i=1}^{m} o_{i}\right)$ to be true.)

Proof: Let $\mathcal{I} \in \mathcal{C}^{R}$ and let $s_{a}=\left\langle o_{1}, \ldots, o_{m}\right\rangle$. There are two cases: either $s_{a}$ is a local state in $\mathcal{I}$, or it is not.

If $s_{a}$ is a local state in $\mathcal{I}$, suppose that $r_{a}(m)=s_{a}$. Note that $\varphi \in \operatorname{Bel}\left(\mathcal{I}, s_{a}\right)$ if and only if $\mathrm{Pl}_{(r, m)}\left(\llbracket \varphi \rrbracket_{(r, m)}\right)>\mathrm{Pl}_{(r, m)}\left(\llbracket \neg \varphi \rrbracket_{(r, m)}\right)$. Recall that, according to the definition of conditioning, $\mathrm{Pl}_{(r, m)}(\cdot)$ is isomorphic to $\mathrm{P} l_{a}\left(\cdot \mid \mathcal{R}\left[\cdot ; o_{1}, \ldots, o_{m}\right]\right)$. Thus, $\mathrm{Pl}_{(r, m)}\left(\llbracket \varphi \rrbracket_{(r, m)}\right)>$ $\mathrm{Pl}_{(r, m)}\left(\llbracket \neg \varphi \rrbracket_{(r, m)}\right)$ if and only if $\mathrm{Pl}_{a}\left(\mathcal{R}[\varphi] \mid \mathcal{R}\left[\cdot ; o_{1}, \ldots, o_{m}\right]\right)>\mathrm{Pl}_{a}\left(\mathcal{R}[\neg \varphi] \mid \mathcal{R}\left[\cdot ; o_{1}, \ldots, o_{m}\right]\right)$. Using $\mathrm{C} 1$, this is true if and only if $\mathrm{Pl}_{a}\left(\mathcal{R}\left[\varphi ; o_{1}, \ldots, o_{m}\right]\right)>\mathrm{Pl}_{a}\left(\mathcal{R}\left[\neg \varphi ; o_{1}, \ldots, o_{m}\right]\right)$. Using REV4, this is true if and only if $\mathrm{Pl}_{a}\left(\mathcal{R}\left[\varphi \wedge \wedge_{i=1}^{m} o_{i}\right]\right)>\mathrm{Pl}_{a}\left(\mathcal{R}\left[\neg \varphi \wedge \wedge_{i=1}^{m} o_{i}\right]\right)$. We get that $\varphi \in \operatorname{Bel}\left(\mathcal{I}, s_{a}\right)$ if and only if $\mathrm{Pl}_{a}\left(\mathcal{R}\left[\varphi \wedge \bigwedge_{i=1}^{m} o_{i}\right]\right)>\mathrm{Pl}_{a}\left(\mathcal{R}\left[\neg \varphi \wedge \bigwedge_{i=1}^{m} o_{i}\right]\right)$. This implies that $\varphi \in \operatorname{Bel}\left(\mathcal{I}, s_{a}\right)$ if and only if $P L_{\mathcal{I}} \models\left(\bigwedge_{i=1}^{m} o_{i}\right) \rightarrow \varphi$.

If $s_{a}$ is not a local state in $\mathcal{I}$, then $\mathcal{R}\left[\cdot ; o_{1}, \ldots, o_{m}\right]=\emptyset$, and by definition $\mathrm{P} l_{a}\left(\mathcal{R}\left[\cdot ; o_{1}, \ldots, o_{m}\right]\right)=$ $\perp$. Using $\mathrm{C} 1$ and REV4, we get that $P L_{a}\left(\mathcal{R}\left[\bigwedge_{i=1}^{m} o_{i}\right]\right)=\perp$, and thus $P L_{\mathcal{I}} \models\left(\bigwedge_{i=1}^{m} o_{i}\right) \rightarrow \varphi$ for all $\varphi \in \mathcal{L}_{e}$. Since $s_{a}$ is not a local state in $\mathcal{I}$, by definition $\operatorname{Bel}\left(\mathcal{I}, s_{a}\right)=\mathcal{L}_{e}$. Hence, we can conclude that $\varphi \in \operatorname{Bel}\left(\mathcal{I}, s_{a}\right)$ if and only if $P L_{\mathcal{I}} \models\left(\bigwedge_{i=1}^{m} o_{i}\right) \rightarrow \varphi$.

We now show that given a ranked plausibility structure $P L$ we can construct a system whose characteristic structure is default-isomorphic to $P L$.

Lemma A.5: Let $P L_{K}=\left(W_{K}, \mathrm{P} 1_{K}, \pi_{K}\right)$ be a plausibility space that satisfies the conditions of Lemma A.3. Then there is a system $\mathcal{I} \in \mathcal{C}^{R}$ such that $P L_{\mathcal{I}}=P L_{K}$. 
Proof: Let $P L_{K}=\left(W_{K}, \mathrm{Pl}_{K}, \pi_{K}\right)$ be a plausibility space that satisfies the conditions of Lemma A.3. For each world $w \in W_{K}$ and sequence of observations $o_{1}, o_{2}, \ldots$, let $r^{w, o_{1}, o_{2}, \ldots}$ be the run defined so that $r_{e}^{w, o_{1}, o_{2}, \ldots}(m)=w$ and $r_{a}^{w, o_{1}, o_{2}, \ldots}(m)=\left\langle o_{1}, \ldots, o_{m}\right\rangle$ for all $m$. Let $\mathcal{R}=\left\{r^{w, o_{1}, o_{2}, \ldots}: \pi_{k}(w)\left(o_{i}\right)=\operatorname{true}\right.$ for all $\left.i\right\}$. Define $\pi$ so that $\pi(r, m)(p)=\pi_{K}\left(r_{e}(m)\right)(p)$ for $p \in \Phi_{e}$, and so that $\pi(r, m)(\operatorname{learn}(\varphi))=$ true if $o_{(r, m)}=\varphi$ for $\varphi \in \mathcal{L}_{e}$. Finally, define the prior plausibility $\mathrm{P}_{a}$ so that $\mathrm{P}_{a}(R)=\mathrm{Pl}_{K}\left(\left\{w: \exists r \in R\left(w=r_{e}(0)\right)\right\}\right.$. It is easy to check that this definition implies that $\mathrm{P} l_{a}(\mathcal{R}[\varphi])=P 1_{K}\left(\llbracket \varphi \rrbracket P L_{K}\right)$. Thus, $P L_{\mathcal{I}}=P L_{K}$. Since $\mathrm{P} 1_{K}$ is a ranking, $\mathrm{P} l_{a}$ is also a ranking and thus qualitative.

We now verify that the resulting interpreted system is indeed in $\mathcal{C}^{R}$. It is easy to check that $\mathcal{I}$ is a belief change system; that is, it satisfies BCS1-BCS5. The construction is such that $r_{e}(m)=r_{e}(0)$ for all runs $r$ and times $m$. Thus, $\mathcal{I}$ satisfies REV1. Since the prior $\mathrm{Pl}_{a}$ is a ranking, this system also satisfies REV2. Lemma A.2 implies that if $\varphi$ is a consistent formula, then $\mathrm{Pl}_{K}\left(\llbracket \varphi \rrbracket_{P L_{K}}\right)>\perp$. This implies that $\mathrm{P} l_{a}(\mathcal{R}[\varphi])>\perp$, and thus the system satisfies REV3. Finally, it is easy to show that $\mathrm{P} l_{a}\left(\mathcal{R}\left[\varphi ; o_{1}, \ldots, o_{m}\right]\right)=$ $\mathrm{Pl}_{a}\left(\mathcal{R}\left[\varphi \wedge o_{1} \wedge \ldots \wedge o_{m}\right]\right)=\mathrm{Pl}_{K}\left(\llbracket \varphi \wedge o_{1} \wedge \ldots \wedge o_{m} \rrbracket P L_{K}\right)$. Thus, the system satisfies REV4.

We are finally ready to prove Theorem 5.2.

Theorem 5.2: Let $\circ$ be an $A G M$ revision operator and let $K \subseteq \mathcal{L}_{e}$ be a consistent belief set. Then there is a system $\mathcal{I}_{(\mathrm{o}, K)} \in \mathcal{C}^{R}$ such that $\operatorname{Bel}\left(\mathcal{I}_{(\circ, K)},\langle\rangle\right)=K$ and

$$
\operatorname{Bel}\left(\mathcal{I}_{(\circ, K)},\langle\rangle\right) \circ \varphi=\operatorname{Bel}\left(\mathcal{I}_{(\circ, K)},\langle\varphi\rangle\right)
$$

for all $\varphi \in \mathcal{L}_{e}$.

Proof: Let $\circ$ be an AGM revision operator and let $K \subseteq \mathcal{L}_{e}$ be a consistent belief set. By Lemmas A.2 and A.5, there is a system $\mathcal{I}_{(\circ, K)}=\left(\mathcal{R}_{(\circ, K)}, \pi_{(\circ, K)}, \mathcal{P}_{(\circ, K)}\right) \in \mathcal{C}^{R}$ such that $P L_{\mathcal{I}_{(0, K)}} \models \varphi \rightarrow \psi$ if and only if $\psi \in K \circ \varphi$. Our construction is such that $\psi \in K \circ \varphi$ if and only if $P L_{\mathcal{I}_{(0, K)}} \models \varphi \rightarrow \psi$. Using Lemma A.4, we get that $P L_{\mathcal{I}_{(0, K)}} \models \varphi \rightarrow \psi$ if and only if $\psi \in \operatorname{Bel}\left(\mathcal{I}_{(\circ, K)},\langle\varphi\rangle\right)$. Thus, $K \circ \varphi=\operatorname{Bel}\left(\mathcal{I}_{(\circ, K)},\langle\varphi\rangle\right)$.

Finally, we show $\operatorname{Bel}\left(\mathcal{I}_{(\circ, K)},\langle\rangle\right)=K$. We start by showing that $K \circ$ true $=K$. Using R3, we get that $K \circ$ true $\subseteq C l(K \cup\{$ true $\})=K$. Since $K$ is consistent, by R $4, C l(K \cup\{$ true $\}) \subseteq$ $K \circ$ true. Thus, $K \circ$ true $=K$. By Lemma A.4, we have that $\operatorname{Bel}(\mathcal{I},\langle\rangle)=\operatorname{Bel}(\mathcal{I},\langle$ true $\rangle)$. Since $\operatorname{Bel}(\mathcal{I},\langle$ true $\rangle)=K \circ$ true, we conclude that $\operatorname{Bel}\left(\mathcal{I}_{(\circ, K)},\langle\rangle\right)=K$.

We next prove Theorem 5.3.

Theorem 5.3: Let $\mathcal{I}$ be a system in $\mathcal{C}^{R}$. Then there is an $A G M$ revision operator ${ }^{\circ} \mathcal{I}$ such that

$$
\operatorname{Bel}\left(\mathcal{I},\langle\rangle \circ_{\mathcal{I}} \varphi=\operatorname{Bel}(\mathcal{I},\langle\varphi\rangle)\right.
$$

for all $\varphi \in \mathcal{L}_{e}$.

Proof: Let $\mathcal{I}=(\mathcal{R}, \pi, \mathcal{P})$ be a system in $\mathcal{C}^{R}$. It is easy to verify that $P L_{\mathcal{I}}$ satisfies the conditions of Lemma A.3 with $K=\operatorname{Bel}(\mathcal{I},\langle\rangle)$. This lemma implies that there is a revision operator $\circ_{\mathcal{I}}$ such that $\psi \in K \circ_{\mathcal{I}} \varphi$ if and only if $P L_{\mathcal{I}} \models \varphi \rightarrow \psi$. Using Lemma A.4, we have that $\psi \in \operatorname{Bel}(\mathcal{I},\langle\varphi\rangle)$ if and only if $\mathrm{Pl}_{\mathcal{I}}=\varphi \rightarrow \psi$. Thus, we have that $K \circ_{\mathcal{I}} \varphi=\operatorname{Bel}(\mathcal{I},\langle\varphi\rangle)$ for all formulas $\varphi$. $\square$ 
Theorem 5.4: Let $\mathcal{I}$ be a system in $\mathcal{C}^{R}$ and $s_{a}=\left\langle o_{1}, \ldots, o_{m}\right\rangle$ be a local state in $\mathcal{I}$. Then there is an $A G M$ revision operator ${ }^{\circ} \mathcal{I}, s_{a}$ such that

$$
\operatorname{Bel}\left(\mathcal{I}, s_{a}\right) \circ_{\mathcal{I}, s_{a}} \varphi=\operatorname{Bel}\left(\mathcal{I}, s_{a} \cdot \varphi\right)
$$

for all formulas $\varphi \in \mathcal{L}_{e}$ such that $o_{1} \wedge \ldots o_{m} \wedge \varphi$ is consistent.

Proof: The structure of the proof is similar to that of Theorem 5.3. As in that proof, we construct a ranked plausibility structure and use Lemma A.3 to find an AGM revision operator. The main difference is that after observing $\varphi_{1}, \ldots, \varphi_{k}$, some events are considered impossible. Lemma A.3, however, requires that all possible formulas are assigned a positive plausibility. We overcome this problem by assigning a "fictional" positive plausibility to all non-empty events that are ruled out by the previous observations.

We proceed as follows. Let $d_{0}$ be a new plausibility value that is less plausible than all positive plausibilities in $\mathrm{Pl}_{a}$; that is, if $\mathrm{Pl}_{a}(A)>\perp$, then $\mathrm{Pl}_{a}(A)>d_{0}$. Let $\mathcal{I}=$ $(\mathcal{R}, \pi, \mathcal{P}) \in \mathcal{C}^{R} ;$ let $s_{a}=\left\langle o_{1}, \ldots, o_{m}\right\rangle$. We define $P L=\left(\mathcal{R}, \mathrm{P} 1, \pi_{P L_{\mathcal{I}}}\right)$, where $\mathrm{Pl}$ is such that $\mathrm{P} 1(\llbracket \varphi \rrbracket)=\max \left(\mathrm{Pl}_{a}\left(\mathcal{R}\left[\varphi \wedge \wedge_{i=1}^{m} o_{i}\right]\right), d_{0}\right)$ for all consistent formulas $\varphi$. This definition implies that if $\varphi$ is consistent with $\bigwedge_{i=1}^{m} o_{i}$, then $\operatorname{Pl}\left(\llbracket \varphi \rrbracket_{P L}\right)=\mathrm{Pl}_{a}\left(\mathcal{R}\left[\varphi_{1} \wedge \bigwedge_{i=1}^{m} o_{i}\right]\right)$.

We now prove that if $\varphi$ is consistent with $\bigwedge_{i=1}^{m} o_{i}$, then $P L \models \varphi \rightarrow \psi$ if and only if $P L_{\mathcal{I}} \models\left(\varphi \wedge \bigwedge_{i=1}^{m} o_{i}\right) \rightarrow \psi$.

For the "if" part, assume that $P L_{\mathcal{I}} \models\left(\varphi \wedge \bigwedge_{i=1}^{m} o_{i}\right) \rightarrow \psi$. Since $\varphi$ is consistent with $\bigwedge_{i=1}^{m} o_{i}$ it follows, from REV3, that $\mathrm{Pl}_{a}\left(\mathcal{R}\left[\varphi \wedge\left(\bigwedge_{i=1}^{m} o_{i}\right]\right)\right)>\perp$. Thus, $\mathrm{Pl}_{a}\left(\mathcal{R}\left[\left(\varphi \wedge\left(\bigwedge_{i=1}^{m} o_{i}\right)\right) \wedge \psi\right]\right)>$ $\mathrm{Pl}_{a}\left(\mathcal{R}\left[\left(\varphi \wedge\left(\bigwedge_{i=1}^{m} o_{i}\right)\right) \wedge \neg \psi\right]\right) \geq \perp$. Thus, $\varphi \wedge \psi$ is consistent with $\bigwedge_{i=1}^{m} o_{i}$. This implies that $\mathrm{P} 1(\llbracket \varphi \wedge \psi \rrbracket)=\mathrm{Pl}_{a}\left(\mathcal{R}\left[\left(\varphi \wedge\left(\bigwedge_{i=1}^{m} o_{i}\right)\right) \wedge \psi\right]>\max \left(d_{0}, \mathrm{Pl}_{a}\left(\mathcal{R}\left[\left(\varphi \wedge\left(\bigwedge_{i=1}^{m} o_{i}\right)\right) \wedge \neg \psi\right]\right)=\right.\right.$ $\mathrm{Pl}(\llbracket \varphi \wedge \neg \psi \rrbracket)$. We conclude that $P L \models \varphi \rightarrow \psi$.

For the "only if" part, assume that $P L_{\mathcal{I}} \forall \forall\left(\varphi \wedge\left(\bigwedge_{i=1}^{m} o_{i}\right)\right) \rightarrow \psi$. This implies that $\mathrm{Pl}_{a}\left(\mathcal{R}\left[\left(\varphi \wedge\left(\bigwedge_{i=1}^{m} o_{i}\right)\right) \wedge \psi\right]\right) \ngtr \mathrm{Pl}_{a}\left(\mathcal{R}\left[\left(\varphi \wedge\left(\bigwedge_{i=1}^{m} o_{i}\right)\right) \wedge \neg \psi\right]\right)$. Since $\mathrm{P} l_{a}$ is a ranking, it follows that $\mathrm{P}_{a}\left(\mathcal{R}\left[\left(\varphi \wedge\left(\bigwedge_{i=1}^{m} o_{i}\right)\right) \psi\right]\right) \leq \mathrm{Pl}_{a}\left(\mathcal{R}\left[\left(\varphi \wedge\left(\bigwedge_{i=1}^{m} o_{i}\right)\right) \wedge \neg \psi\right]\right)$. Since $\perp<\mathrm{Pl}_{a}(\mathcal{R}[\varphi \wedge$ $\left.\left.\left(\bigwedge_{i=1}^{m} o_{i}\right)\right]\right)=\max \left(\mathrm{Pl}_{a}\left(\mathcal{R}\left[\left(\varphi \wedge\left(\bigwedge_{i=1}^{m} o_{i}\right)\right) \wedge \psi\right]\right), \mathrm{Pl}_{a}\left(\mathcal{R}\left[\left(\varphi \wedge\left(\bigwedge_{i=1}^{m} o_{i}\right)\right) \wedge \neg \psi\right]\right)\right)$, we have that $\mathrm{Pl}_{a}\left(\mathcal{R}\left[\left(\varphi \wedge\left(\bigwedge_{i=1}^{m} o_{i}\right)\right) \wedge \neg \psi\right]\right)>\perp$. We conclude that $\operatorname{Pl}(\llbracket \varphi \wedge \neg \psi \rrbracket) \geq \operatorname{Pl}(\llbracket \varphi \wedge \psi \rrbracket)$. Thus, $P L \forall \forall \rightarrow \psi$.

It is easy to verify that $P L$ is ranked, and satisfies the requirements of Lemma A.3. Thus, there exists a revision operator ${ }^{\circ} \mathcal{I}, s_{a}$ such that $\psi \in K{ }^{\circ}{\mathcal{I}, s_{a}} \varphi$ if and only if $P L \models \varphi \rightarrow \psi$, where $K=\{\varphi: P L \models$ true $\rightarrow \varphi\}$. Moreover, since for all $\varphi$ consistent with $\bigwedge_{i=1}^{m} o_{i}$ we have that $P L \models \varphi \rightarrow \psi$ if and only if $P L_{\mathcal{I}} \models\left(\varphi \wedge\left(\bigwedge_{i=1}^{m} o_{i}\right)\right) \rightarrow \psi$, then, from Lemma A.4, it follows that $K=\operatorname{Bel}\left(\mathcal{I}, s_{a}\right)$ and that if $\varphi$ is consistent with $\bigwedge_{i=1}^{m} o_{i}$, then $P L \models \varphi \rightarrow \psi$ if and only if $\psi \in \operatorname{Bel}\left(\mathcal{I}, s_{a} \cdot \varphi\right)$.

Theorem 5.5: Let $\mathcal{I}$ be a system in $\mathcal{C}^{R}$ whose local states are $\mathcal{E}_{\mathcal{L}_{e}}$. There is a function

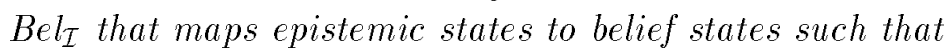

- if $s_{a}$ is a local state of the agent in $\mathcal{I}$, then $\operatorname{Bel}\left(\mathcal{I}, s_{a}\right)=\operatorname{Be} l_{\mathcal{I}}\left(s_{a}\right)$, and

- $\left(\circ, B e l_{\mathcal{I}}\right)$ satisfies $R 1^{\prime}-R 8^{\prime}$.

Proof: As we said earlier, roughly speaking, we define $\operatorname{Bel}_{\mathcal{I}}\left(s_{a}\right)=\operatorname{Bel}\left(\mathcal{I}, s_{a}\right)$ when $s_{a}$ is a local state in $\mathcal{I}$. If $s_{a}$ is not in $\mathcal{I}$, then we set $\operatorname{Bel}_{\mathcal{I}}\left(s_{a}\right)=\operatorname{Bel}\left(\mathcal{I}, s^{\prime}\right)$, where $s^{\prime}$ is the longest 
consistent suffix of $s_{a}$. We now make this definition precise, and show that the resulting $\mathrm{Bel}_{\mathcal{I}}$ satisfies $\mathrm{R} 1^{\prime}-\mathrm{R} 8^{\prime}$.

We proceed as follows. We define a function $f(\cdot)$ that maps sequences of observations to suffixes as follows:

$$
f\left(\left\langle o_{1}, \ldots, o_{m}\right\rangle\right)= \begin{cases}\langle\rangle & \text { if } m=0, \\ \langle\text { false }\rangle & \text { if } m>0 \text { and } o_{m} \text { is inconsistent, } \\ \left\langle o_{k}, \ldots, o_{m}\right\rangle & \text { otherwise, with } k \leq m \text { the minimal index } \\ & \text { s. t. } \forall \mathcal{L}_{e} \neg\left(o_{k} \wedge \ldots \wedge o_{m}\right) .\end{cases}
$$

Aside from the special case where $o_{m}$ is inconsistent, we simply choose the longest suffix of $s_{a}$ that is still consistent. We define $\operatorname{Bel}_{\mathcal{I}}\left(s_{a}\right)=\operatorname{Bel}\left(\mathcal{I}, f\left(s_{a}\right)\right)$. Clearly, if $s_{a}$ is a local state in $\mathcal{I}$, then $f\left(s_{a}\right)=s_{a}$, so $\operatorname{Bel}_{\mathcal{I}}\left(s_{a}\right)=\operatorname{Bel}\left(\mathcal{I}, s_{a}\right)$.

We now have to show that $\left(\circ, \operatorname{Be} l_{\mathcal{I}}\right)$ satisfies $\mathrm{R} 1^{\prime}-\mathrm{R} 8^{\prime}$. The proof outline is as follows. Given a particular state $s_{a}$, we construct a ranked plausibility structure that corresponds, in the sense of Lemma A.2, to belief change from $s_{a}$. We then use Lemma A.3 to show that belief changes from $s_{a}$ satisfies the AGM postulates, i.e., R1-R8. Since this is true from any $s_{a}$, we get that $\mathrm{Bel}_{\mathcal{I}}$ satisfies $\mathrm{R} 1^{\prime}-\mathrm{R} 8^{\prime}$.

Let $s_{a}=\left\langle o_{1}, \ldots, o_{m}\right\rangle$. We define a ranked plausibility space that has the following structure. The most plausible events are the ones consistent with $o_{1} \wedge \ldots \wedge o_{m}$. They are ordered according to the prior ranking conditioned on $o_{1} \wedge \ldots \wedge o_{m}$. The next tier of events are those that are inconsistent with $o_{1} \wedge \ldots \wedge o_{m}$ but are consistent $o_{2} \wedge \ldots \wedge o_{m}$. Again, these are ordered according to the prior ranking conditioned on $o_{2} \wedge \ldots \wedge o_{m}$. We continue this way; the last tier consists of all events that are inconsistent with $\boldsymbol{o}_{m}$.

Formally, let $P L=\left(\mathcal{R}, \mathrm{P} 1, \pi_{P L_{\mathcal{T}}}\right)$, where $\mathrm{P} 1$ is such that $\mathrm{P} 1(\llbracket \varphi \rrbracket) \geq \mathrm{P} 1(\llbracket \psi \rrbracket)$ if $\mathrm{P}_{a}(\mathcal{R}[\varphi \wedge$ $\left.\left.\left(\bigwedge_{i=k}^{m} o_{i}\right]\right)\right) \geq \mathrm{Pl}_{a}\left(\mathcal{R}\left[\psi \wedge\left(\bigwedge_{i=k}^{m} o_{i}\right]\right)\right)$ where $k \leq m+1$ is the greatest integer such that for all $j<k, \varphi$ and $\psi$ are both inconsistent with $\bigwedge_{i=j}^{m} o_{i}$. It is easy to see that $P L$ is ranked, and that if $\varphi$ is consistent, then $\mathrm{Pl}(\llbracket \varphi \rrbracket)>\perp$.

Let $\varphi \in \mathcal{L}_{e}$. We now show that $P L \models \varphi \rightarrow \psi$ if and only if $\psi \in \operatorname{Bel}_{\mathcal{I}}\left(s_{a} \cdot \varphi\right)$. If $\varphi$ is inconsistent, then $P L \models \varphi \rightarrow \psi$ for all $\psi$. Moreover, since $\varphi$ is inconsistent, $f\left(s_{a} \cdot \varphi\right)=\langle$ false $\rangle$, and thus $\operatorname{Bel}_{\mathcal{I}}\left(s_{a} \cdot \varphi\right)=\mathcal{L}_{e}$. We conclude that $\varphi \rightarrow \psi$ if and only if $\psi \in \operatorname{Bel}_{\mathcal{I}}\left(s_{a} \cdot \varphi\right)$. If $\varphi$ is consistent, then let $k \leq m+1$ be the greatest integer such that for all $j<k, \varphi$ is inconsistent with $\bigwedge_{i=j}^{m} o_{i}$. It is easy to verify that $f\left(s_{a} \cdot \varphi\right)=\left\langle o_{k}, \ldots, o_{m}, \varphi\right\rangle$. From Lemma A.4, it follows that $\psi \in \operatorname{Bel}_{\mathcal{I}}\left(s_{a} \cdot \varphi\right)=\operatorname{Bel}\left(\mathcal{I},\left\langle o_{k}, \ldots, o_{m}, \varphi\right\rangle\right)$ if and only if $\operatorname{Pl}_{a}\left(\mathcal{R}\left[\left(\varphi \wedge\left(\bigwedge_{i=k}^{m} o_{i}\right)\right) \wedge \psi\right]\right)>$ $\mathrm{Pl}_{a}\left(\mathcal{R}\left[\left(\varphi \wedge\left(\bigwedge_{i=k}^{m} o_{i}\right)\right) \wedge \neg \psi\right]\right)$. We now show that this is the case if and only if $P L \models \varphi \rightarrow \psi$. Suppose that $P L_{a}\left(\mathcal{R}\left[\left(\varphi \wedge \wedge\left(\bigwedge_{i=k}^{m} o_{i}\right)\right) \wedge \psi\right]\right)>P L_{a}\left(\mathcal{R}\left[\left(\varphi \wedge\left(\bigwedge_{i=k}^{m} o_{i}\right)\right) \wedge \neg \psi\right]\right)$. Then, clearly, $\mathrm{Pl}_{a}\left(\mathcal{R}\left[\left(\varphi \wedge\left(\bigwedge_{i=k}^{m} o_{i}\right)\right) \wedge \psi\right]\right)>\perp$, and thus $\varphi \wedge \psi$ is consistent with $o_{k}, \ldots, o_{m}$. Since both $\varphi \wedge \psi$ and $\varphi \wedge \neg \psi$ are inconsistent with $o_{j}, \ldots, o_{m}$ for all $j<k$, we have that $\operatorname{Pl}(\llbracket \varphi \wedge \psi \rrbracket)>$ $\mathrm{Pl}(\llbracket \varphi \wedge \neg \psi \rrbracket)$. On other hand, if $\mathrm{Pl}_{a}\left(\mathcal{R}\left[\left(\varphi \wedge\left(\bigwedge_{i=k}^{m} o_{i}\right)\right) \wedge \psi\right]\right) \ngtr \mathrm{Pl}_{a}\left(\mathcal{R}\left[\left(\varphi \wedge\left(\bigwedge_{i=k}^{m} o_{i}\right)\right) \wedge \neg \psi\right]\right)$, then since $\mathrm{Pl}_{a}$ is a ranking $P L_{a}\left(\mathcal{R}\left[\left(\varphi \wedge\left(\bigwedge_{i=k}^{m} o_{i}\right)\right) \wedge \psi\right]\right) \leq P L_{a}\left(\mathcal{R}\left[\left(\varphi \wedge\left(\bigwedge_{i=k}^{m} o_{i}\right)\right) \wedge \neg \psi\right]\right)$. Moreover, since $\varphi$ is consistent with $o_{k} \wedge \ldots \wedge o_{m}$, we have that $\operatorname{Pl}\left(\mathcal{R}\left[\varphi \wedge\left(\bigwedge_{i=k}^{m} o_{i}\right)\right]\right)>\perp$. This implies that $\mathrm{Pl}_{a}\left(\mathcal{R}\left[\left(\varphi \wedge\left(\bigwedge_{i=k}^{m} o_{i}\right)\right) \wedge \neg \psi\right]\right)>\perp$ and thus $\mathrm{Pl}(\llbracket \varphi \wedge \psi \rrbracket) \leq \operatorname{Pl}(\llbracket \varphi \wedge \neg \psi \rrbracket)$. We conclude that $P L \models \varphi \rightarrow \psi$ if and only if $\psi \in \operatorname{Bel}_{\mathcal{I}}\left(s_{a} \cdot \varphi\right)$.

By Lemma A.3, there is a revision operator $\circ_{s_{a}}$ that satisfies $\mathrm{R} 1-\mathrm{R} 8$ such that $\psi \in K \circ \varphi$ if and only if $P L \models \varphi \rightarrow \psi$. It is not hard to check that this implies that the change from $\operatorname{Bel}_{\mathcal{I}}\left(s_{a}\right)$ to $\operatorname{Bel}_{\mathcal{I}}\left(s_{a} \cdot \varphi\right)$ satisfies $\mathrm{R} 1^{\prime}-\mathrm{R} 8^{\prime}$. 
Proposition 5.6: Let $\mathcal{I}$ be a system in $\mathcal{C}^{R}$ whose local states are $\mathcal{E}_{\mathcal{L}_{e}}$. There is a function Bel $l_{\mathcal{I}}$ that maps epistemic states to belief states such that

- if $s_{a}$ is a local state of the agent in $\mathcal{I}$, then $\operatorname{Bel}\left(\mathcal{I}, s_{a}\right)=\operatorname{Be} l_{\mathcal{I}}\left(s_{a}\right)$, and

- $\left(\circ, B e l_{\mathcal{I}}\right)$ satisfies $R t^{\prime}-R g^{\prime}$.

Proof: As we said in the main text, we show that the function $\mathrm{Bel}_{\mathcal{I}}$ defined in the proof of Theorem 5.5 satisfies $\mathrm{R} 9{ }^{\prime}$. Let $s_{a}=\left\langle o_{1}, \ldots, o_{m}\right\rangle$, and let $\varphi, \psi \in \mathcal{L}_{e}$ be formulas such that $\forall_{\mathcal{L}_{e}} \neg(\varphi \wedge \psi)$. Since $\varphi$ is consistent with $\psi$, we get that $f\left(s_{a} \cdot \varphi \cdot \psi\right)=\left\langle o_{k}, \ldots, o_{m}, \varphi, \psi\right\rangle$, where $k \leq m$ is the least integer such that $\varphi \wedge \psi$ is consistent with $o_{k}, \ldots, o_{m}$. For the same reason, we get that $f\left(s_{a} \cdot \varphi \wedge \psi\right)=\left\langle o_{k}, \ldots, o_{m}, \varphi \wedge \psi\right\rangle$. Using Lemma A.4 we immediately get that $\operatorname{Bel}\left(\mathcal{I},\left\langle o_{k}, \ldots, o_{m}, \varphi, \psi\right\rangle\right)=\operatorname{Bel}\left(\mathcal{I},\left\langle o_{k}, \ldots, o_{m}, \varphi \wedge \psi\right\rangle\right)$. Thus, we conclude that $\operatorname{Bel}_{\mathcal{I}}\left(s_{a} \cdot \varphi \cdot \psi\right)=\operatorname{Bel}_{\mathcal{I}}\left(s_{a} \cdot \varphi \wedge \psi\right)$.

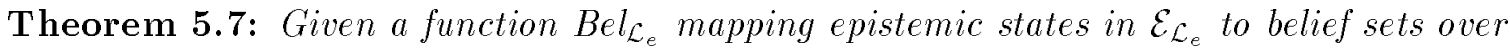
$\mathcal{L}_{e}$ such that Bel $\mathcal{L}_{\mathcal{L}_{e}}(\langle\rangle)$ is consistent and $\left(\right.$ Bel $\left._{\mathcal{L}_{e}}, \circ\right)$ satisfies $R 1^{\prime}-R g^{\prime}$, there is a system $\mathcal{I} \in \mathcal{C}^{R}$ whose local states are in $\mathcal{E}_{\mathcal{L}_{e}}$ such that $\operatorname{Bel}_{\mathcal{L}_{e}}\left(s_{a}\right)=\operatorname{Bel}\left(\mathcal{I}, s_{a}\right)$ for each local state $s_{a}$ in $\mathcal{I}$.

Proof: We show that $\operatorname{Bel}\left(\mathcal{I}, s_{a}\right)=\operatorname{Bel}_{\mathcal{L}_{e}}\left(s_{a}\right)$ for local states $s_{a}$ in $\mathcal{I}$, where $\mathcal{I}$ is the system guaranteed to exist by Theorem 5.2 such that $\operatorname{Bel}(\mathcal{I},\langle\rangle)=\operatorname{Bel}_{\mathcal{L}_{e}}(\langle\rangle)$ and $\operatorname{Bel}(\mathcal{I},\langle\varphi\rangle)=$ $\operatorname{Bel}_{\mathcal{L}_{e}}(\langle\varphi\rangle)$ for all $\varphi \in \mathcal{L}_{e}$. We prove this by induction on the length $m$ of $s_{a}$. For $m \leq 1$, this is true by our choice of $\mathcal{I}$. For the induction case, let $s_{a}=\left\langle o_{1}, \ldots, o_{m}\right\rangle$ be a local state in $\mathcal{I}$. Thus, $o_{1} \wedge \ldots \wedge o_{m}$ is consistent. From R9', it follows that $\operatorname{Bel}_{\mathcal{L}_{e}}\left(\left\langle o_{1}, \ldots, o_{m}\right\rangle\right)=\operatorname{Bel}_{\mathcal{L}_{e}}\left(\left\langle o_{1}, \ldots, o_{m-2}, o_{m-1} \wedge o_{m}\right\rangle\right)$. Using the induction hypothesis, we have that $\operatorname{Bel}_{\mathcal{L}_{e}}\left(\left\langle o_{1}, \ldots, o_{m-2}, o_{m-1} \wedge o_{m}\right\rangle\right)=\operatorname{Bel}\left(\mathcal{I},\left\langle o_{1}, \ldots, o_{m-2}, o_{m-1} \wedge o_{m}\right\rangle\right)$. Using Lemma A.4, we get that $\operatorname{Bel}\left(\mathcal{I},\left\langle o_{1}, \ldots, o_{m-2}, o_{m-1} \wedge o_{m}\right\rangle\right)=\operatorname{Bel}\left(\mathcal{I},\left\langle o_{1}, \ldots, o_{m}\right\rangle\right)$. Thus, we conclude that $\operatorname{Bel}_{\mathcal{L}_{e}}\left(\left\langle o_{1}, \ldots, o_{m}\right\rangle\right)=\operatorname{Bel}\left(\mathcal{I},\left\langle o_{1}, \ldots, o_{m}\right\rangle\right)$. $\square$

\section{A.2 Proofs for Section 6}

In this section we prove Theorem 6.2 . We now show that any system in $\mathcal{C}^{U}$ corresponds to an update structure. Suppose that $\mathcal{I}=(\mathcal{R}, \pi, \mathcal{P}) \in \mathcal{C}^{U}$ is such that the set of environment states is $\mathcal{S}_{e}$ and the prior of BCS5 is consistent with distance function $d$. Define an update structure $U_{\mathcal{I}}=\left(S_{e}, \pi_{e}, d\right)$, where for $p \in \Phi_{e}, \pi_{e}\left(s_{e}\right)(p)=\pi\left(\left(s_{e}, s_{a}\right)\right)(p)$ for some choice of $s_{a}$. By BCS1, the choice of $s_{a}$ does not matter. It is easy to see that UPD1 ensures that $S_{e}$ and $\pi_{e}$ satisfy the requirements of the definition of update structures. We want to show that belief change in $\mathcal{I}$ corresponds to belief change in $U_{\mathcal{I}}$ in the sense of Theorem 6.1 . Since Theorem 6.1 states that any belief change operation defined by an update structure satisfies U1-U8, this will suffice to prove the "if" direction of Theorem 6.2. To prove the "only if" direction of Theorem 6.2 , we show that that for any update structure $U$, there is a system $\mathcal{I} \in \mathcal{C}^{U}$ such that $U_{\mathcal{I}}=U$.

We start with preliminary definitions and lemmas for the "if" direction of Theorem 6.2. Let $s_{a}=\left\langle o_{1}, \ldots, o_{m}\right\rangle$. We define $\operatorname{States}\left(\mathcal{I}, s_{a}\right)=\left\{s \in \mathcal{S}_{e}: s \models \xi\right.$ for all $\left.\xi \in \operatorname{Bel}\left(\mathcal{I}, s_{a}\right)\right\}$. Clearly, if $\varphi$ is such that $\operatorname{Bel}\left(\mathcal{I}, s_{a}\right)=C l(\varphi)$, then $\operatorname{States}\left(\mathcal{I}, s_{a}\right)=\llbracket \varphi \rrbracket U_{\mathcal{I}}$. To show that belief change in $\mathcal{I}$ corresponds to belief change in $U_{\mathcal{I}}$ we have to show that

$$
\operatorname{States}\left(\mathcal{I}, s_{a} \cdot \psi\right)=\min _{U_{\mathcal{I}}}\left(\operatorname{States}\left(\mathcal{I}, s_{a}\right), \llbracket \psi \rrbracket_{U_{\mathcal{I}}}\right) .
$$


This is proved in Lemma A.8. To prove this lemma, we need some preliminary lemmas.

Lemma A.6: Let $\mathcal{I} \in \mathcal{C}^{U}$, and let $s_{a}=\left\langle o_{1}, \ldots, o_{m}\right\rangle$. Then $\varphi \in \operatorname{Bel}\left(\mathcal{I}, s_{a}\right)$ if and only if $(\mathcal{I}, r, 0) \models\left(\bigcirc o_{1} \wedge \ldots \wedge \bigcirc^{m} o_{m}\right) \rightarrow \bigcirc^{m} \varphi$ for some run $r$ in $\mathcal{R}$.

Proof: The proof of this lemma is analogous to the proof of Lemma A.4, using UPD3 and UPD4 instead of REV3 and REV4. We do not repeat the argument here.

We now provide an alternative characterization of States $\left(\mathcal{I}, s_{a}\right)$ in terms of the agent's prior on run-prefixes.

Lemma A.7: Let $\mathcal{I} \in \mathcal{C}^{U}$ and let $s_{a}=\left\langle o_{1}, \ldots, o_{m}\right\rangle$. Then $s_{m} \in \operatorname{States}\left(\mathcal{I}, s_{a}\right)$ if and only if there is a sequence of states $\left[s_{0}, \ldots, s_{m}\right] \subseteq \mathcal{R}\left[\right.$ true, $\left.o_{1}, \ldots, o_{m}\right]$ such that $\mathrm{Pl}_{a}\left(\left[s_{0}, \ldots, s_{m}\right]\right) \nless$ $\mathrm{P}_{a}\left(\mathcal{R}\left[\right.\right.$ true $\left.\left., o_{1}, \ldots, o_{m}\right] \Leftrightarrow\left[s_{0}, \ldots, s_{m}\right]\right)$.

Proof: For the "if" direction, assume that there is a sequence $s_{0}, \ldots, s_{m}$ such that $\left[s_{0}, \ldots, s_{m}\right] \subseteq \mathcal{R}\left[\right.$ true $\left., o_{1}, \ldots, o_{m}\right]$, and $\mathrm{Pl}_{a}\left(\left[s_{0}, \ldots, s_{m}\right]\right) \quad \nless \quad \mathrm{Pl}_{a}\left(\mathcal{R}\left[\right.\right.$ true $\left., o_{1}, \ldots, o_{m}\right] \Leftrightarrow$ $\left.\left[s_{0}, \ldots, s_{m}\right]\right)$. By way of contradiction, assume that $s_{m} \notin \operatorname{States}(\mathcal{I}, m)$. Thus, there is a formula $\xi \in \operatorname{Bel}(\mathcal{I}, m)$ such that $s_{m} \models-\xi$. From Lemma A.6 it follows that since $\xi \in \operatorname{Bel}\left(\mathcal{I}, s_{a}\right)$, $(\mathcal{I}, r, 0) \models\left(\bigcirc o_{1} \wedge \ldots \wedge \bigcirc^{m} o_{m}\right) \rightarrow \bigcirc^{m} \xi$ for some run $r$ in $\mathcal{R}$. From the definition of conditioning it follows that $\mathrm{P}_{a}\left(\mathcal{R}\left[\right.\right.$ true $\left.\left., o_{1}, \ldots, o_{m-1}, o_{m} \wedge \xi\right]\right)>\mathrm{Pl}_{a}\left(\mathcal{R}\left[\right.\right.$ true $\left.\left., o_{1}, \ldots, o_{m-1}, o_{m} \wedge \neg \xi\right]\right)$. Since $s_{m} \models \neg \xi$, we get that $\left[s_{0}, \ldots, s_{m}\right] \subseteq \mathcal{R}\left[\right.$ true $\left., o_{1}, \ldots, o_{m-1}, o_{m} \wedge \neg \xi\right]$ and that $\mathcal{R}\left[\right.$ true $\left., o_{1}, \ldots, o_{m-1}, o_{m} \wedge \xi\right] \subseteq \mathcal{R}\left[\right.$ true $\left., o_{1}, \ldots, o_{m}\right] \Leftrightarrow\left[s_{0}, \ldots, s_{m}\right]$. From A1, it follows that $\mathrm{Pl}_{a}\left(\left[s_{0}, \ldots, s_{m}\right]\right)<\mathrm{Pl}_{a}\left(\mathcal{R}\left[\right.\right.$ true $\left.\left., o_{1}, \ldots, o_{m}\right] \Leftrightarrow\left[s_{0}, \ldots, s_{m}\right]\right)$, which contradicts our starting assumption. We conclude that $s_{m} \in \operatorname{States}\left(\mathcal{I}, s_{a}\right)$.

For the "only if" direction, assume that $s_{m} \in \operatorname{States}(\mathcal{I}, a)$. Since $\mathcal{S}_{e}$ is finite and $\pi_{e}$ assigns a different truth assignment to each state in $\mathcal{S}_{e}$, there is a formula $\xi \in \mathcal{L}_{e}$ that characterizes $s_{m}$; that is, $s \models \xi$ if and only if $s=s_{m}$. Since $s_{m} \in \operatorname{States}\left(\mathcal{I}, s_{a}\right)$, we have that $\neg \xi \notin \operatorname{Bel}\left(\mathcal{I}, s_{a}\right)$. Using Lemma A.6, we get that $(\mathcal{I}, r, 0) \forall\left(\bigcirc o_{1} \wedge \ldots \wedge \bigcirc^{m} o_{m}\right) \rightarrow \bigcirc^{m} \neg \xi$ for all runs $r \in \mathcal{R}$. By BCS5, this is true if and only if $\mathrm{Pl}_{a}\left(\mathcal{R}\left[\right.\right.$ true, $\left.\left.o_{1}, \ldots, o_{m}\right]\right)>\perp$ and $\mathrm{Pl}_{a}\left(\mathcal{R}\left[\right.\right.$ true $\left.\left., o_{1}, \ldots, o_{m-1}, o_{m} \wedge \xi\right]\right) \nless \mathrm{Pl}_{a}\left(\mathcal{R}\left[\right.\right.$ true $\left.\left., o_{1}, \ldots, o_{m-1}, o_{m} \wedge \neg \xi\right]\right)$. By UPD2, there is a sequence $\left[s_{0}, \ldots, s_{m}\right] \subseteq \mathcal{R}\left[\right.$ true $\left., o_{1}, \ldots, o_{m-1}, o_{m} \wedge \xi\right]$ such that $\mathrm{Pl}_{a}\left(\left[s_{0}, \ldots, s_{m}\right]\right) \nless$ $\mathrm{P}_{a}\left(\left[s_{0}^{\prime}, \ldots, s_{m}^{\prime}\right]\right)$ for all $\left[s_{0}^{\prime}, \ldots, s_{m}^{\prime}\right] \subseteq \mathcal{R}\left[\right.$ true $\left., o_{1}, \ldots, o_{m-1}, o_{m} \wedge \neg \xi\right]$. Moreover, without loss of generality, we can assume that $\mathrm{P} l_{a}\left(\left[s_{0}, \ldots, s_{m}\right]\right) \nless \mathrm{Pl}_{a}\left(\left[s_{0}^{\prime}, \ldots, s_{m}^{\prime}\right]\right)$ for all $\left[s_{0}^{\prime}, \ldots, s_{m}^{\prime}\right] \subseteq$ $\mathcal{R}\left[\right.$ true $\left., o_{1}, \ldots, o_{m-1}, o_{m} \wedge \xi\right]$, since there are only finitely many such sequences. Thus, by $\mathrm{UPD} 2, \mathrm{Pl}_{a}\left(\left[s_{0}, \ldots, s_{m}\right]\right) \nless \mathrm{Pl}_{a}\left(\mathcal{R}\left[\right.\right.$ true $\left.\left., o_{1}, \ldots, o_{m}\right] \Leftrightarrow\left[s_{0}, \ldots, s_{m}\right]\right)$.

We can now prove that belief change in $\mathcal{I}$ corresponds to belief change in $U_{\mathcal{I}}$.

Lemma A.8: Let $\mathcal{I}=(\mathcal{R}, \pi, \mathcal{P}) \in \mathcal{C}^{U}$ Then

$$
\operatorname{States}\left(\mathcal{I}, s_{a} \cdot \psi\right)=\min _{U_{\mathcal{I}}}\left(\operatorname{States}\left(\mathcal{I}, s_{a}\right), \llbracket \psi \rrbracket U_{\mathcal{I}}\right)
$$

for all local states $s_{a}$ and formulas $\psi \in \mathcal{L}_{e}$.

Proof: Let $\mathrm{P} 1_{a}$ be the prior in $\mathcal{I}$; assume that $\mathrm{P} 1_{a}$ consistent with a distance function $d$. Let $s_{a}=\left\langle o_{1}, \ldots, o_{m}\right\rangle$. 
To show that $\min _{U_{\mathcal{I}}}\left(\operatorname{States}\left(\mathcal{I}, s_{a}\right), \llbracket \psi \rrbracket_{U_{\mathcal{I}}}\right) \subseteq \operatorname{States}\left(\mathcal{I}, s_{a} \cdot \psi\right)$, suppose that $s \in$ $\min _{U_{\mathcal{I}}}\left(\operatorname{States}\left(\mathcal{I}, s_{a}\right), \llbracket \psi \rrbracket_{U_{\mathcal{I}}}\right)$. Thus, there is a state $s_{m} \in \operatorname{States}\left(\mathcal{I}, s_{a}\right)$ such that $d\left(s_{m}, s^{\prime}\right) \nless$ $d\left(s_{m}, s\right)$ for all states $s^{\prime}$ that satisfy $\psi$. We want to show that $s \in \operatorname{States}\left(\mathcal{I}, s_{a}\right.$. $\psi$ ). From Lemma A.7, it follows that, since $s_{m} \in \operatorname{States}\left(\mathcal{I}, s_{a}\right)$, there is a sequence $s_{0}, \ldots, s_{m-1}$ such that $\left[s_{0}, \ldots, s_{m}\right] \in \mathcal{R}\left[\right.$ true $\left., o_{1}, \ldots, o_{m-1}, o_{m}\right]$ and $\mathrm{Pl}_{a}\left(\left[s_{0}, \ldots, s_{m}\right]\right) \nless$ $\mathrm{Pl}_{a}\left(\mathcal{R}\left[\right.\right.$ true $\left.\left., o_{1}, \ldots, o_{m-1}, o_{m}\right] \Leftrightarrow\left[s_{0}, \ldots, s_{m}\right]\right)$. We now show that $\mathrm{Pl}_{a}\left(\left[s_{0}, \ldots, s_{m}, s\right]\right) \nless$ $\mathrm{Pl}_{a}\left(\mathcal{R}\left[\right.\right.$ true $\left.\left., o_{1}, \ldots, o_{m}, \psi\right] \Leftrightarrow\left[s_{0}, \ldots, s_{m}, s\right]\right)$. By Lemma A.7, this suffices to show that $s \in \operatorname{States}\left(\mathcal{I}, s_{a} \cdot \psi\right)$. Suppose that $\left[s_{0}^{\prime}, \ldots, s_{m+1}^{\prime}\right] \subseteq \mathcal{R}\left[\operatorname{true}, o_{1}, \ldots, o_{m}, \psi\right] \Leftrightarrow\left[s_{0}, \ldots, s_{m}, s\right]$. If $\left[s_{0}, \ldots, s_{m}\right]=\left[s_{0}^{\prime}, \ldots, s_{m}^{\prime}\right]$, then we have that $d\left(s_{m}^{\prime}, s_{m+1}^{\prime}\right) \nless d\left(s_{m}, s\right)$. Since $\mathrm{P}_{a}$ is consistent with $d$, it follows that $\mathrm{Pl}_{a}\left(\left[s_{0}, \ldots, s_{m}, s\right]\right) \nless \mathrm{Pl}_{a}\left(\left[s_{0}^{\prime}, \ldots, s_{m}^{\prime}, s_{m+1}^{\prime}\right]\right)$. If $\left[s_{0}, \ldots, s_{m}\right] \neq$ $\left[s_{0}^{\prime}, \ldots, s_{m}^{\prime}\right]$, then, since $\mathrm{Pl}_{a}\left(\left[s_{0}, \ldots, s_{m}\right]\right) \nless \mathrm{Pl}_{a}\left(\left[s_{0}^{\prime}, \ldots, s_{m}^{\prime}\right]\right)$ and $\mathrm{Pl}_{a}$ is consistent with $d$, we have that $\mathrm{Pl}_{a}\left(\left[s_{0}, \ldots, s_{m}, s\right]\right) \nless \mathrm{Pl}_{a}\left(\left[s_{0}^{\prime}, \ldots, s_{m}^{\prime}, s_{m+1}^{\prime}\right]\right)$.

Since $\quad \mathrm{Pl}_{a}\left(\left[s_{0}, \ldots, s_{m}, s\right]\right) \quad \nless \quad \mathrm{Pl}_{a}\left(\left[s_{0}^{\prime}, \ldots, s_{m}^{\prime}, s_{m+1}^{\prime}\right]\right)$ for all $\left[s_{0}^{\prime}, \ldots, s_{m+1}^{\prime}\right] \subseteq \mathcal{R}\left[\right.$ true $\left., o_{1}, \ldots, o_{m}, \psi\right] \Leftrightarrow\left[s_{0}, \ldots, s_{m}, s\right]$ and $\mathrm{P} l_{a}$ is prefix-defined, we have that $\mathrm{Pl}_{a}\left(\left[s_{0}, \ldots, s_{m}, s\right]\right) \nless \mathrm{Pl}_{a}\left(\mathcal{R}\left[\right.\right.$ true $\left., o_{1}, \ldots, o_{m}, \psi\right] \Leftrightarrow\left[s_{0}, \ldots, s_{m}, s\right]$. By Lemma A.7, $s \in \operatorname{States}\left(\mathcal{I}, s_{a} \cdot \psi\right)$, as desired.

To show that $\operatorname{States}\left(\mathcal{I}, s_{a} \cdot \psi\right) \subseteq \min _{U_{\mathcal{I}}}\left(\operatorname{States}\left(\mathcal{I}, s_{a}\right), \llbracket \psi \rrbracket_{U_{\mathcal{I}}}\right)$, suppose that $s \in$ $\operatorname{States}\left(\mathcal{I}, s_{a} \cdot \psi\right)$. By Lemma A.7, there is a sequence $s_{0}, \ldots, s_{m}$ such that $\left.\left[s_{0}, \ldots, s_{m}, s\right]\right) \subseteq$ $\mathcal{R}\left[\right.$ true $\left., o_{1}, \ldots, o_{m}, \psi\right]$ and $\mathrm{P}_{a}\left(\left[s_{0}, \ldots, s_{m}, s\right]\right) \nless \mathrm{Pl}_{a}\left(\mathcal{R}\left[\right.\right.$ true $\left.\left., o_{1}, \ldots, o_{m}, \psi\right] \Leftrightarrow\left[s_{0}, \ldots, s_{m}, s\right]\right)$. We want to show that $s_{m} \in \operatorname{States}\left(\mathcal{I}, s_{a}\right)$ and that $d\left(s_{m}, s^{\prime}\right) \nless d\left(s_{m}, s\right)$ for all $s^{\prime}$ that satisfy $\psi$. This suffices to prove that $s \in \min _{U_{\mathcal{I}}}\left(\operatorname{States}\left(\mathcal{I}, s_{a}\right), \llbracket \psi \rrbracket_{U_{\mathcal{I}}}\right)$.

To show that $s_{m} \in \operatorname{States}\left(\mathcal{I}, s_{a}\right)$, by Lemma A.7, it suffices to show that $\mathrm{Pl}_{a}\left(\left[s_{0}, \ldots, s_{m}\right]\right) \nless$ $\mathrm{P}_{a}\left(\mathcal{R}\left[\right.\right.$ true $\left.\left., o_{1}, \ldots, o_{m}\right] \Leftrightarrow\left[s_{0}, \ldots, s_{m}\right]\right)$. Let $s_{0}^{\prime}, \ldots, s_{m}^{\prime}$ be a sequence such that $\left[s_{0}^{\prime}, \ldots, s_{m}^{\prime}\right] \subseteq$ $\mathcal{R}\left[\right.$ true $\left., o_{1}, \ldots, o_{m}\right]$. By definition, $\left[s_{0}^{\prime}, \ldots, s_{m}^{\prime}, s\right] \subseteq \mathcal{R}\left[\right.$ true $\left., o_{1}, \ldots, o_{m}, \psi\right]$. Thus, from our choice of $s_{0}, \ldots, s_{m}$, it follows that $\mathrm{Pl}_{a}\left(\left[s_{0}, \ldots, s_{m}, s\right]\right) \nless \mathrm{Pl}_{a}\left(\left[s_{0}^{\prime}, \ldots, s_{m}^{\prime}, s\right]\right)$. Since $\mathrm{Pl}_{a}$ is consistent with $d$, it follows that $\mathrm{Pl}_{a}\left(\left[s_{0}, \ldots, s_{m}\right]\right) \nless \mathrm{Pl}_{a}\left(\left[s_{0}^{\prime}, \ldots, s_{m}^{\prime}\right]\right)$. Thus, by Lemma A.7, $s_{m} \in \operatorname{States}\left(\mathcal{I}, s_{a}\right)$. To see that $d\left(s_{m}, s^{\prime}\right) \nless d\left(s_{m}, s\right)$ for all $s^{\prime}$ that satisfy $\psi$, let $s^{\prime} \neq s$ be such that $s^{\prime} \models \psi$. Thus, $\left[s_{0}, \ldots, s_{m}, s^{\prime}\right] \subseteq\left[\right.$ true $\left., o_{1}, \ldots, o_{m}, \psi\right]$. From our choice of $s_{0}, \ldots, s_{m}$, it follows that $\mathrm{P} l_{a}\left(\left[s_{0}, \ldots, s_{m}, s\right]\right) \nless \mathrm{P}_{a}\left(\left[s_{0}, \ldots, s_{m}, s^{\prime}\right]\right)$. Since $\mathrm{Pl}_{a}$ is consistent with $d$, it follows that $d\left(s_{m}, s^{\prime}\right) \nless d\left(s_{m}, s\right)$. We conclude that $s \in \min _{U_{\mathcal{I}}}\left(\operatorname{States}\left(\mathcal{I}, s_{a}\right), \llbracket \psi \rrbracket_{U_{\mathcal{I}}}\right)$.

We now have the tools to prove the "if" direction of Theorem 6.2.

Lemma A.9: If $\mathcal{I}=(\mathcal{R}, \pi, \mathcal{P}) \in \mathcal{C}^{U}$, then there is a belief change operator $\diamond$ that satisfies U1-U8 such that

$$
\operatorname{Bel}\left(\mathcal{I}, s_{a}\right) \diamond \psi=\operatorname{Bel}\left(\mathcal{I}, s_{a} \cdot \psi\right)
$$

for all local states $s_{a}$ and formulas $\psi \in \mathcal{L}_{e}$.

Proof: Let $\mathcal{I} \in \mathcal{C}^{U}$. Using the arguments we presented above, it easy to check that $U_{\mathcal{I}}$ is an update structure. By Theorem 6.1, there is a belief change operator $\diamond$ that satisfies U1-U8 such that $\llbracket \varphi \diamond \psi \rrbracket_{U_{\mathcal{I}}}=\min _{U_{\mathcal{I}}}\left(\llbracket \varphi \rrbracket_{U_{\mathcal{I}}}, \llbracket \psi \rrbracket_{U_{\mathcal{I}}}\right)$ for all $\varphi, \psi \in \mathcal{L}_{e}$. From Lemma A.8, it follows that $\operatorname{Bel}\left(\mathcal{I}, s_{a}\right) \diamond \psi=\operatorname{Bel}\left(\mathcal{I}, s_{a} \cdot \psi\right)$.

We now prove the "only if" direction of Theorem 6.2. Suppose that $\diamond$ is a belief change operator that satisfies U1-U8. According to Theorem 6.1, there is an update structure $U_{\diamond}$ that corresponds to $\diamond$. Thus, it suffices to show that there is a system $\mathcal{I}$ such that $U_{\mathcal{I}}=U_{\diamond}$. 
Lemma A.10: Let $U=\left(W, d, \pi_{U}\right)$ be an update structure. Then there is a system $\mathcal{I} \in \mathcal{C}^{U}$ such that $U_{\mathcal{I}}=U$.

Proof: Given the sequences $w_{0}, w_{1}, \ldots \in W$ and $o_{1}, o_{2}, \ldots \in \mathcal{L}_{e}$, let $r^{w_{0}, w_{1}, \ldots ; o_{1}, o_{2}, \ldots}$ be the run defined so that $r_{e}^{w_{0}, w_{1}, \ldots ; o_{1}, o_{2}, \ldots}(m)=w_{m}$ and $r_{a}^{w_{0}, w_{1}, \ldots ; o_{1}, o_{2}, \ldots}(m)=\left\langle o_{1}, \ldots, o_{m}\right\rangle$. Let $\mathcal{R}=\left\{r^{w_{0}, w_{1}, \ldots ; o_{1}, o_{2}, \ldots}: \pi_{U}\left(w_{m}\right)\left(o_{m}\right)=\right.$ true for all $\left.m\right\}$. Define $\pi$ such that $\pi(r, m)(p)=$ $\pi_{U}\left(r_{e}(m)\right)(p)$ for $p \in \Phi_{e}$ and $\pi(r, m)(\operatorname{learn}(\varphi))=\operatorname{true}$ if $o_{(r, m)}=\varphi$ for $\varphi \in \mathcal{L}_{e}$.

It is clear that $(\mathcal{R}, \pi)$ satisfies BCS1-BCS4 and UPD1. Thus, all that remains to show is that there is a prior plausibility measure $\mathrm{Pl}_{a}$ that satisfies UPD2-UPD4. This will ensure that $(\mathcal{R}, \pi, \mathcal{P}) \in \mathcal{C}^{U}$.

We proceed as follows. We define a preferential space $(\mathcal{R}, \prec)$ where $r \prec r^{\prime}$ if and only if there is some $m$ such that $r_{e}(k)=r_{e}^{\prime}(k)$ for all $0 \leq k \leq m, r_{e}(m+1) \neq r_{e}^{\prime}(m+1)$, and $d\left(r_{e}(m), r_{e}(m+1)\right)<d\left(r_{e}^{\prime}(m), r_{e}^{\prime}(m+1)\right)$. Recall that $r \prec r^{\prime}$ denotes that $r$ is preferred over $r^{\prime}$. Thus, this ordering is consistent with the comparison of events of the form $\left[s_{0}, \ldots, s_{n}\right]$ according to UPD2.

Using the construction of Proposition 2.2, there is a plausibility space $\left(R, \mathrm{P} l_{a}\right)$ such that $\mathrm{Pl}_{a}(A) \geq \mathrm{Pl}_{a}(B)$ if and only if for all $r \in B \Leftrightarrow A$, there is a run $r^{\prime} \in A$ such that $r^{\prime} \prec r$ and there is no $r^{\prime \prime} \in B \Leftrightarrow A$ such that $r^{\prime \prime} \prec r^{\prime}$. By (Friedman \& Halpern, 1998b, Theorem 5.5), $\mathrm{P} l_{a}$ is a qualitative plausibility measure. We now show that it satisfies UPD2-UPD4.

We start with UPD2. To show that $\mathrm{Pl}_{A}$ is consistent with $d$, we need to show that $\mathrm{Pl}_{a}\left(\left[s_{0}, \ldots, s_{n}\right]\right)<\mathrm{Pl}_{a}\left(\left[s_{0}^{\prime}, \ldots, s_{n}^{\prime}\right]\right)$ if and only if there is some $m<n$ such that $s_{k}=s_{k}^{\prime}$ for all $0 \leq k \leq m$, and $d\left(s_{m}, s_{m+1}\right)>d\left(s_{m}^{\prime}, s_{m+1}^{\prime}\right)$. Suppose that $\mathrm{Pl}_{a}\left(\left[s_{0}, \ldots, s_{n}\right]\right)<$ $\mathrm{Pl}_{a}\left(\left[s_{0}^{\prime}, \ldots, s_{n}^{\prime}\right]\right)$. Let $r$ be some run in $\left[s_{0}, \ldots,{ }_{n}^{\prime}\right]$. Without loss of generality we can assume that $r_{e}(m)=r_{e}(n)$ for all $m>n$. Since $\mathrm{Pl}_{a}\left(\left[s_{0}, \ldots, s_{n}\right]\right)<\mathrm{Pl}_{a}\left(\left[s_{0}^{\prime}, \ldots, s_{n}^{\prime}\right]\right)$, there is a run $r^{\prime} \in\left[s_{0}^{\prime}, \ldots, s_{n}^{\prime}\right]$ such that $r^{\prime} \prec r$. By definition, this implies that there is an $m$ such that $r_{e}(k)=r_{e}^{\prime}(k)$ for all $0 \leq k \leq m$, and $d\left(r_{e}^{\prime}(m), r_{e}^{\prime}(m+1)\right)<d\left(r_{e}(m), r_{e}(m+1)\right)$. We claim that $m<n$. For if $m \geq n$, then $r_{e}(m+1)=r_{e}(m)$ by construction, so $d\left(r_{e}(m), r_{e}(m+1)\right)=$ $d\left(r_{e}(m), r_{e}(m)\right) \leq d\left(r_{e}^{\prime}(m), r_{e}^{\prime}(m+1)\right)$ and $r^{\prime} \nprec r$, a contradiction. Thus, $s_{k}=s_{k}^{\prime}$ for all $0 \leq k \leq m, d\left(s_{m}^{\prime}, s_{m+1}^{\prime}\right)<d\left(s_{m}, s_{m+1}\right)$.

For the converse, suppose that there is an $m<n$ such that $s_{k}=s_{k}^{\prime}$ for all $0 \leq k \leq m$, and $d\left(s_{m}^{\prime}, s_{m+1}^{\prime}\right)<d\left(s_{m}, s_{m+1}\right)$. Let $r^{\prime}$ be the run where $r_{e}^{\prime}(k)=s_{k}^{\prime}$ for $k \leq n, r_{e}^{\prime}(k)=s_{n}^{\prime}$ for $k \geq n$, and $o_{\left(r^{\prime}, k\right)}=$ true for all $k$. It follows $r^{\prime} \prec r$ for all runs $r^{\prime} \in\left[s_{0}, \ldots, s_{n}\right]$. Thus, $\mathrm{Pl}_{a}\left(\left[s_{0}, \ldots, s_{n}\right]\right)<\mathrm{Pl}_{a}\left(\left[s_{0}^{\prime}, \ldots, s_{n}^{\prime}\right]\right)$.

To show that $\mathrm{P} l_{a}$ is prefix-defined, we must show that $\mathrm{P} l_{a}\left(\mathcal{R}\left[\varphi_{0}, \ldots, \varphi_{n}\right]\right) \geq \mathrm{P}_{a}\left(\mathcal{R}\left[\psi_{0}, \ldots, \psi_{n}\right]\right)$ if and only if for all $\left[s_{0}, \ldots, s_{n}\right] \subseteq \mathcal{R}\left[\psi_{0}, \ldots, \psi_{n}\right] \Leftrightarrow \mathcal{R}\left[\varphi_{0}, \ldots, \varphi_{n}\right]$, there is some $\left[s_{0}^{\prime}, \ldots, s_{n}^{\prime}\right] \subseteq$ $\mathcal{R}\left[\varphi_{0}, \ldots, \varphi_{n}\right]$ such that $\mathrm{P}_{a}\left(\left[s_{0}^{\prime}, \ldots, s_{n}^{\prime}\right]\right)>\mathrm{Pl}_{a}\left(\left[s_{0}, \ldots, s_{n}\right]\right)$. Suppose that $\mathrm{Pl}_{a}\left(\mathcal{R}\left[\varphi_{0}, \ldots, \varphi_{n}\right]\right) \geq$ $\mathrm{Pl}_{a}\left(\mathcal{R}\left[\psi_{0}, \ldots, \psi_{n}\right]\right)$. Let $\left[s_{0}, \ldots, s_{n}\right] \subseteq \mathcal{R}\left[\psi_{0}, \ldots, \psi_{n}\right] \Leftrightarrow \mathcal{R}\left[\varphi_{0}, \ldots, \varphi_{n}\right]$. Let $r \in\left[s_{0}, \ldots, s_{n}\right]$ be a run such that $r_{e}(m)=r_{e}(n)$ for all $m \geq n$. Since $\mathrm{Pl}_{a}\left(\mathcal{R}\left[\varphi_{0}, \ldots, \varphi_{n}\right]\right) \geq \mathrm{Pl}_{a}\left(\mathcal{R}\left[\psi_{0}, \ldots, \psi_{n}\right]\right)$ there is a run $r^{\prime} \in \mathcal{R}\left[\varphi_{0}, \ldots, \varphi_{n}\right]$ such that $r^{\prime} \prec r$. This implies that there is an $m$ such that $r_{e}(k)=r_{e}^{\prime}(k)$ for all $0 \leq k \leq m$, and $d\left(r_{e}^{\prime}(m), r_{e}^{\prime}(m+1)\right)<d\left(r_{e}(m), r_{e}(m+1)\right)$. As before, we have that $m<n$, and thus $\mathrm{Pl}_{a}\left(\left[r_{e}^{\prime}(0), \ldots, r_{e}^{\prime}(n)\right]\right)>\mathrm{Pl}_{a}\left(\left[s_{0}, \ldots, s_{n}\right]\right)$. Since $r^{\prime} \in \mathcal{R}\left[\varphi_{0}, \ldots, \varphi_{n}\right]$, we also have that $\left[r_{e}^{\prime}(0), \ldots, r_{e}^{\prime}(n)\right] \subseteq \mathcal{R}\left[\varphi_{0}, \ldots, \varphi_{n}\right]$, as desired.

For the converse, assume that for all $\left[s_{0}, \ldots, s_{n}\right] \subseteq \mathcal{R}\left[\psi_{0}, \ldots, \psi_{n}\right] \Leftrightarrow \mathcal{R}\left[\varphi_{0}, \ldots, \varphi_{n}\right]$ there is some $\left[s_{0}^{\prime}, \ldots, s_{n}^{\prime}\right] \subseteq \mathcal{R}\left[\varphi_{0}, \ldots, \varphi_{n}\right]$ such that $\mathrm{Pl}_{a}\left(\left[s_{0}^{\prime}, \ldots, s_{n}^{\prime}\right]\right)>\mathrm{Pl}_{a}\left(\left[s_{0}, \ldots, s_{n}\right]\right)$. This implies that $\mathrm{Pl}_{a}\left(\mathcal{R}\left[\varphi_{0}, \ldots, \varphi_{n}\right]\right)>\mathrm{Pl}_{a}\left(\left[s_{0}, \ldots, s_{n}\right]\right)$ for all for all $\left[s_{0}, \ldots, s_{n}\right] \subseteq \mathcal{R}\left[\psi_{0}, \ldots, \psi_{n}\right] \Leftrightarrow$ 
$\mathcal{R}\left[\varphi_{0}, \ldots, \varphi_{n}\right]$. Since there are only finitely many sequences of states of length $m$, we can apply $\mathrm{A} 2$, and conclude that $\mathrm{Pl}_{a}\left(\mathcal{R}\left[\varphi_{0}, \ldots, \varphi_{n}\right]\right)>\mathrm{Pl}_{a}\left(\mathcal{R}\left[\psi_{0}, \ldots, \psi_{n}\right] \Leftrightarrow \mathcal{R}\left[\varphi_{0}, \ldots, \varphi_{n}\right]\right)$. Thus, $\mathrm{Pl}_{a}\left(\mathcal{R}\left[\varphi_{0}, \ldots, \varphi_{n}\right]\right) \geq \mathrm{Pl}_{a}\left(\left(\mathcal{R}\left[\psi_{0}, \ldots, \psi_{n}\right]\right)\right)$.

For UPD3, recall that the construction of Proposition 2.2 is such that $\mathrm{Pl}_{a}(R)>\perp$ for all non-empty $R \subseteq \mathcal{R}$. Since, by our construction, the set $\mathcal{R}\left[\varphi_{0}, \ldots, \varphi_{n}\right]$ is non-empty for all sequences $\varphi_{0}, \ldots, \varphi_{n}$ of consistent formulas, UPD3 must hold.

Finally, we consider UPD4. We have to show that $\mathrm{Pl}_{a}\left(\mathcal{R}\left[\varphi_{0}, \ldots, \varphi_{n+1} ; o_{1}, \ldots, o_{n}\right]\right) \geq$ $\mathrm{Pl}_{a}\left(\mathcal{R}\left[\psi_{0}, \ldots, \psi_{n+1} ; o_{1}, \ldots, o_{n}\right]\right)$ if and only if $\mathrm{P} l_{a}\left(\mathcal{R}\left[\varphi_{0}, \varphi_{1} \wedge o_{1}, \ldots, \varphi_{n} \wedge o_{n}, \varphi_{n+1}\right]\right) \geq \mathrm{Pl}_{a}\left(\mathcal{R}\left[\psi_{0}, \psi_{1} \wedge\right.\right.$ $\left.\left.o_{1}, \ldots, \psi_{n} \wedge o_{n}, \psi_{n+1}\right]\right)$. By construction, $\mathcal{R}\left[\varphi_{0}, \ldots, \varphi_{n+1} ; o_{1}, \ldots, o_{n}\right] \subseteq \mathcal{R}\left[\varphi_{0}, \varphi_{1} \wedge o_{1}, \ldots, \varphi_{n} \wedge\right.$ $\left.o_{n}, \varphi_{n+1}\right]$. On the other hand, for each run $r \in \mathcal{R}\left[\varphi_{0}, \varphi_{1} \wedge o_{1}, \ldots, \varphi_{n} \wedge o_{n}, \varphi_{n+1}\right]$ there is a run $r^{\prime} \in \mathcal{R}\left[\varphi_{0}, \ldots, \varphi_{n+1} ; o_{1}, \ldots, o_{n}\right]$ such that $r_{e}^{\prime}(m)=r_{e}(m)$ for all $m$, and $o_{(r, m)}=o_{m}$ for $1 \leq m \leq n$. Since the preference ordering on runs is a function only of the environment states, it is clear that $r$ and $r^{\prime}$ are compared in the same manner; that is for all $r^{\prime \prime}, r^{\prime \prime} \prec r$ if and only if $r^{\prime \prime} \prec r^{\prime}$, and $r \prec r^{\prime \prime}$ if and only if $r^{\prime} \prec r^{\prime \prime}$. Thus, we conclude that for the purposes of the preference ordering, both $\mathcal{R}\left[\varphi_{0}, \varphi_{1} \wedge o_{1}, \ldots, \varphi_{n} \wedge o_{n}, \varphi_{n+1}\right]$ and $\mathcal{R}\left[\varphi_{0}, \ldots, \varphi_{n+1} ; o_{1}, \ldots, o_{n}\right]$ are compared in the same manner to other sets. It easy to see that this suffices to show that $\mathrm{Pl}_{a}$ satisfies UPD4.

Finally, we can prove Theorem 6.2 .

Theorem 6.2: A belief change operator $\diamond$ satisfies U1-U8 if and only if there is a system $\mathcal{I} \in \mathcal{C}^{U}$ such that

$$
\operatorname{Bel}\left(\mathcal{I}, s_{a}\right) \diamond \psi=\operatorname{Bel}\left(\mathcal{I}, s_{a} \cdot \psi\right)
$$

for all epistemic states $s_{a}$ and formulas $\psi \in \mathcal{L}_{e}$.

Proof: The "if" direction follows from Lemma A.9. For the "only if" direction, assume that $\diamond$ satisfies U1-U8. By Theorem 6.1, there is an update structure $U_{\diamond}$ such that $\llbracket \varphi \diamond \psi \rrbracket_{U_{\mathcal{I}}}=$ $\min _{U_{\mathcal{T}}}\left(\llbracket \varphi \rrbracket_{U_{\mathcal{T}}}, \llbracket \psi \rrbracket_{U_{\mathcal{T}}}\right)$ for all $\varphi, \psi \in \mathcal{L}_{e}$. By Lemma A.10, there is a system $\mathcal{I} \in \mathcal{C}^{U}$ such that $U_{\mathcal{I}}=U_{\diamond}$. From Lemma A.8, it follows that $\operatorname{Bel}\left(\mathcal{I}, s_{a}\right) \diamond \psi=\operatorname{Bel}\left(\mathcal{I}, s_{a} \cdot \psi\right)$ for all local states $s_{a}$ and formulas $\psi \in \mathcal{L}_{e}$.

\section{References}

Alchourrón, C. E., Gärdenfors, P., \& Makinson, D. (1985). On the logic of theory change: partial meet functions for contraction and revision. Journal of Symbolic Logic, 50, $510-530$.

Boutilier, C. (1992). Normative, subjective and autoepistemic defaults: adopting the Ramsey test. In Principles of Knowledge Representation and Reasoning: Proc. Third International Conference (KR '92), pp. 685-696. Morgan Kaufmann, San Francisco, Calif.

Boutilier, C. (1994a). Conditional logics of normality: a modal approach. Artificial Intelligence, $68,87-154$.

Boutilier, C. (1994b). Unifying default reasoning and belief revision in a modal framework. Artificial Intelligence, 68, 33-85. 
Boutilier, C. (1996a). Iterated revision and minimal change of conditional beliefs. Journal of Philosophical Logic, 25, 262-305.

Boutilier, C. (1996b). Abduction to plausible causes: An event-based model of belief update. Artificial Intelligence, 83, 143-166.

Boutilier, C. (1998). A unified model of qualitative belief change: A dynamical systems perspective. Artificial Intelligence, 98, 281-316.

Boutilier, C., Friedman, N., \& Halpern, J. Y. (1998). Belief revision with unreliable observations. In Proceedings, Fifteenth National Conference on Artificial Intelligence (AAAI'96), pp. 127-134.

Burgess, J. (1981). Quick completeness proofs for some logics of conditionals. Notre Dame Journal of Formal Logic, 22, 76-84.

Darwiche, A., \& Pearl, J. (1997). On the logic of iterated belief revision. Artificial Intelligence, $89,1-29$.

Davis, R., \& Hamscher, W. (1988). Model-based reasoning: troubleshooting. In Shrobe, H., \& for Artificial Intelligence, T. A. A. (Eds.), Exploring AI, pp. 297-346. Morgan Kaufmann, SF.

de Rijke, M. (1992). Meeting some neighbors. Research report LP-92-10, University of Amsterdam.

del Val, A., \& Shoham, Y. (1992). Deriving properties of belief update from theories of action. In Proceedings, Tenth National Conference on Artificial Intelligence (AAAI 92), pp. 584-589. AAAI Press, Menlo Park, CA.

del Val, A., \& Shoham, Y. (1993). Deriving properties of belief update from theories of action (II). In Proc. Thirteenth International Joint Conference on Artificial Intelligence (IJCAI'93), pp. 732-737 San Francisco. Morgan Kaufmann.

del Val, A., \& Shoham, Y. (1994). A unified view of belief revision and update. Journal of Logic and Computation, 4.

Dubois, D., \& Prade, H. (1990). An introduction to possibilistic and fuzzy logics. In Shafer, G., \& Pearl, J. (Eds.), Readings in Uncertain Reasoning, pp. 742-761. Morgan Kaufmann, San Francisco, Calif.

Dubois, D., \& Prade, H. (1991). Possibilistic logic, preferential models, non-monotonicity and related issues. In Proc. Twelfth International Joint Conference on Artificial Intelligence (IJCAI'91), pp. 419-424. Morgan Kaufmann, San Francisco.

Fagin, R., Halpern, J. Y., Moses, Y., \& Vardi, M. Y. (1995). Reasoning about Knowledge. MIT Press, Cambridge, Mass.

Freund, M., \& Lehmann, D. (1994). Belief revision and rational inference. Tech. rep. TR 94-16, Hebrew University. 
Friedman, N. (1997). Modeling Beliefs in Dynamic Systems. Ph.D. thesis, Stanford.

Friedman, N., \& Halpern, J. Y. (1994). Conditional logics of belief change. In Proc. National Conference on Artificial Intelligence (AAAI'94), pp. 915-921. AAAI Press, Menlo Park, CA.

Friedman, N., \& Halpern, J. Y. (1995). Plausibility measures: a user's manual. In Besnard, P., \& Hanks, S. (Eds.), Proc. Eleventh Conference on Uncertainty in Artificial Intelligence (UAI'95), pp. 175-184. Morgan Kaufmann, San Francisco.

Friedman, N., \& Halpern, J. Y. (1996). A qualitative Markov assumption and its implications for belief change. In Proc. Twelfth Conference on Uncertainty in Artificial Intelligence (UAI'96), pp. 263-273.

Friedman, N., \& Halpern, J. Y. (1997). Modeling belief in dynamic systems. part I: foundations. Artificial Intelligence, 95(2), 257-316.

Friedman, N., \& Halpern, J. Y. (1998a). Belief revision: A critique. Journal of Logic, Language and Information, To appear. Also available at http://www.cs.huji.ac. il/ ^nir. A preliminary version appeared in L. C. Aiello, J. Doyle, and S. C. Shapiro (eds.) Principles of Knowledge Representation and Reasoning: Proc. 5'th International Conference, pp. 421-431, 1996.

Friedman, N., \& Halpern, J. Y. (1998b). Plausibility measures and default reasoning. Journal of the ACM, To appear. Also available at http://ww.huji.ac.il/“nir. A preliminary version appeared in Proc., $13^{\prime}$ th National Conference on Artificial Intelligence, pp. 1297-1304, 1996.

Fuhrmann, A. (1989). Reflective modalities and theory change. Synthese, 81, 115-134.

Gärdenfors, P. (1986). Belief revision and the Ramsey test for conditionals. Philosophical Review, 91, 81-93.

Gärdenfors, P. (1988). Knowledge in Flux. MIT Press, Cambridge, Mass.

Gärdenfors, P., \& Makinson, D. (1988). Revisions of knowledge systems using epistemic entrenchment. In Proc. Second Conference on Theoretical Aspects of Reasoning about Knowledge, pp. 83-95. Morgan Kaufmann, San Francisco, Calif.

Goldszmidt, M., Morris, P., \& Pearl, J. (1993). A maximum entropy approach to nonmonotonic reasoning. IEEE Transactions of Pattern Analysis and Machine Intelligence, $15(3), 220-232$.

Goldszmidt, M., \& Pearl, J. (1996). Qualitative probabilities for default reasoning, belief revision, and causal modeling. Artificial Intelligence, 84, 57-112.

Grahne, G., Mendelzon, A., \& Rieter, R. (1992). On the semantics of belief revision systems. In Moses, Y. (Ed.), know92, pp. 132-142. Morgan Kaufmann, San Francisco, Calif.

Grove, A. (1988). Two modelings for theory change. Journal of Philosophical Logic, 17, $157-170$. 
Halpern, J. Y., \& Fagin, R. (1989). Modelling knowledge and action in distributed systems. Distributed Computing, 3(4), 159-179. A preliminary version appeared in Proc. 4th ACM Symposium on Principles of Distributed Computing, 1985, with the title "A formal model of knowledge, action, and communication in distributed systems: preliminary report".

Halpern, J. Y., \& Vardi, M. Y. (1989). The complexity of reasoning about knowledge and time, I: lower bounds. Journal of Computer and System Sciences, 38 (1), 195-237.

Katsuno, H., \& Mendelzon, A. (1991a). On the difference between updating a knowledge base and revising it. In Principles of Knowledge Representation and Reasoning: Proc. Second International Conference (KR '91), pp. 387-394. Morgan Kaufmann, San Francisco, Calif.

Katsuno, H., \& Mendelzon, A. (1991b). Propositional knowledge base revision and minimal change. Artificial Intelligence, 52(3), 263-294.

Katsuno, H., \& Satoh, K. (1991). A unified view of consequence relation, belief revision and conditional logic. In Proc. Twelfth International Joint Conference on Artificial Intelligence (IJCAI'91), pp. 406-412.

Kautz, H. A. (1986). Logic of persistence. In Proceedings, Fifth National Conference on Artificial Intelligence (AAAI'86), pp. 401-405. AAAI Press, Menlo Park, CA.

Keller, A. M., \& Winslett, M. (1985). On the use of an extended relational model to handle changing incomplete information. IEEE Transactions on Software Engineering, SE$11(7), 620-633$.

Kraus, S., Lehmann, D., \& Magidor, M. (1990). Nonmonotonic reasoning, preferential models and cumulative logics. Artificial Intelligence, 4f, 167-207.

Lehmann, D. (1995). Belief revision, revised. In Proc. Fourteenth International Joint Conference on Artificial Intelligence (IJCAI'95), pp. 1534-1540. Morgan Kaufmann, San Francisco.

Levi, I. (1988). Iteration of conditionals and the Ramsey test. Synthese, 76, 49-81.

Lewis, D. K. (1973). Counterfactuals. Harvard University Press, Cambridge, Mass.

Manna, Z., \& Pnueli, A. (1992). The Temporal Logic of Reactive and Concurrent Systems, Vol. 1. Springer-Verlag, Berlin/New York.

Nayak, A. C. (1994). Iterated belief change based on epistemic entrenchment. Erkenntnis, 41, 353-390.

Pearl, J. (1989). Probabilistic semantics for nonmonotonic reasoning: a survey. In Brachman, R. J., Levesque, H. J., \& Reiter, R. (Eds.), Proc. First International Conference on Principles of Knowledge Representation and Reasoning (KR '89), pp. 505-516. Reprinted in Readings in Uncertain Reasoning, G. Shafer and J. Pearl (eds.), Morgan Kaufmann, San Francisco, Calif., 1990, pp. 699-710. 
Rott, H. (1991). Two methods of constructing contractions and revisions of knowledge systems. Journal of Philosophical Logic, 20, 149-173.

Rott, H. (1992). Two methods of constructing contraction and revisions of knowledge systems. Journal of Logic, Language and Information, 1, 45-78.

Shafer, G. (1976). A Mathematical Theory of Evidence. Princeton University Press, Princeton, N.J.

Shoham, Y. (1987). A semantical approach to nonmonotonic logics. In Proc. 2nd IEEE Symp. on Logic in Computer Science, pp. 275-279. Reprinted in M. L. Ginsberg (Ed.), Readings in Nonmonotonic Reasoning, Morgan Kaufman, San Francisco, Calif., 1987, pp. $227-250$.

Shoham, Y. (1988). Chronological ignorance: experiments in nonmonotonic temporal reasoning. Artificial Intelligence, 36, 271-331.

Spohn, W. (1988). Ordinal conditional functions: a dynamic theory of epistemic states. In Harper, W., \& Skyrms, B. (Eds.), Causation in Decision, Belief Change, and Statistics, Vol. 2, pp. 105-134. Reidel, Dordrecht, Netherlands.

Wang, Z., \& Klir, G. J. (1992). Fuzzy Measure Theory. Plenum Press, New York.

Williams, M. (1994). Transmutations of knowledge systems. In Principles of Knowledge Representation and Reasoning: Proc. Fourth International Conference (KR '94), pp. 619-629. Morgan Kaufmann, San Francisco, Calif.

Winslett, M. (1988). Reasoning about action using a possible models approach. In Proceedings, Seventh National Conference on Artificial Intelligence (AAAI'88), pp. 89-93. AAAI Press, Menlo Park, CA. 